\title{
Isostructural Charge-Transfer Co-crystals based on Triptycene End-Capped Quinoxalinophenanthrophenazine
}

Lucas Ueberricke, ${ }^{[a, b]}$ Farhad Ghalami, ${ }^{[c]}$ Wen-Shan Zhang, ${ }^{[\mathrm{d}, \mathrm{b}]}$ Vaishnavi Rao, ${ }^{[\mathrm{e}, \mathrm{b}]}$ Frank Rominger, ${ }^{[\mathrm{a}]}$ Rasmus R. Schröder, [d,b] Marcus Elstner, ${ }^{[c]}$ Michael Mastalerz ${ }^{*}[a, b]$

\section{-Supporting Information-}

[a] Organisch-Chemisches Institut, Ruprecht-Karls-Universität Heidelberg Im Neuenheimer Feld 270, 69120 Heidelberg (Germany)

Email: Michael.mastalerz@oci.uni-heidelberg.de

[b] Centre for Advanced Materials (CAM), Ruprecht-Karls-Universität Heidelberg

Im Neuenheimer Feld 225, 69120 Heidelberg (Germany)

[c] Institut für Physikalische Chemie, Karlsruher Institute of Technology (KIT)

Kaiserstr. 12, 76131 Karlsruhe (Germany)

[d] BioQuant, Ruprecht-Karls-Universität Heidelberg

Im Neuenheimer Feld 267, 69120 Heidelberg

[e] Institut für Physikalische Chemie, Ruprecht-Karls-Universität Heidelberg

Im Neuenheimer Feld 253, 69120 Heidelberg (Germany)

\section{Content}

1 Experimental Section

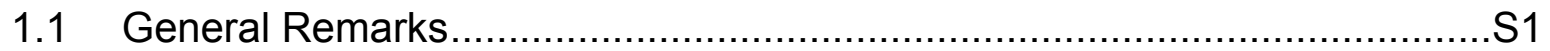

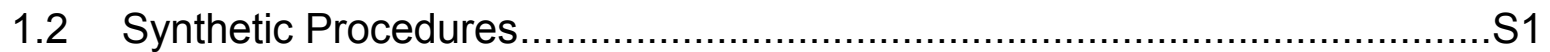

1.3 Sample preparation of Charge Transfer Complexes ................................S2

2 Optical Characterization of Thin-Films ........................................................ 4

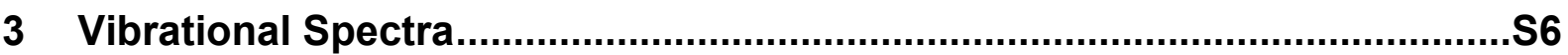

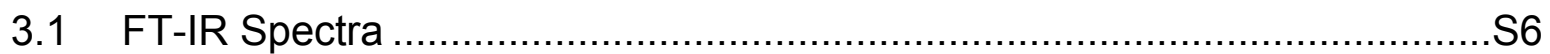

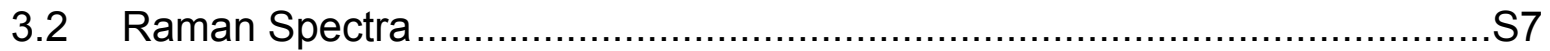

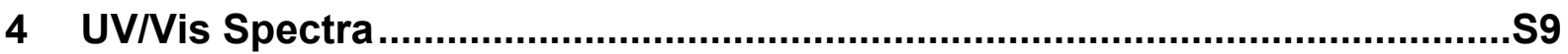

5 Crystal Structure Analysis ..................................................................... 9

5.1 Determination of Distances and Displacement of $\pi$ Stacked Planes ...........S9

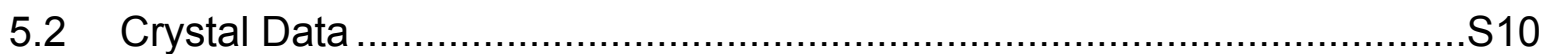

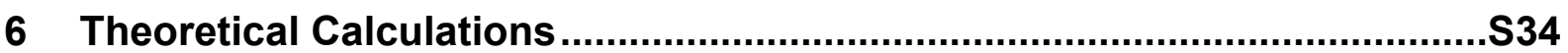

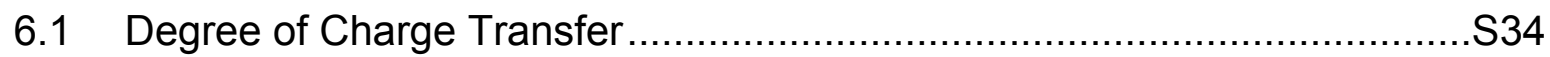

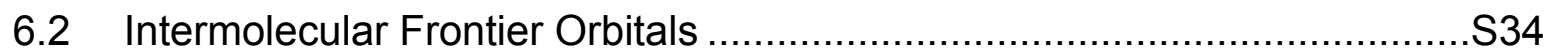

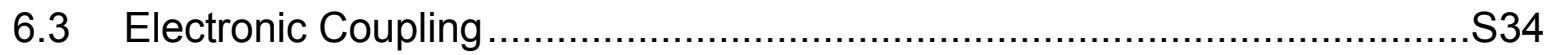

6.3.1 Direct Coupling of $\pi$ Stacked Dimers (DFTB) ..............................S34

6.3.2 Superexchange Coupling (Effective Transfer Integrals) ....................S36

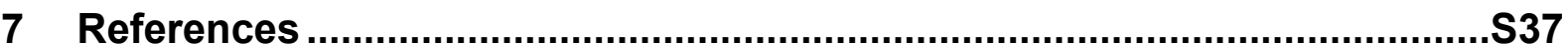




\section{Experimental Section}

\subsection{General Remarks}

All reagents and solvents were purchased from Fisher Scientific, Alfa Aesar, SigmaAldrich, TCl or VWR and were used without further purification unless otherwise noted. NMR spectra were recorded by using Bruker Avance DRX (300 MHz). IR spectra were recorded on a ZnSe ATR crystal by using a Bruker Lumos spectrometer with a resolution of $4 \mathrm{~cm}^{-1}$. The charge sensitive $\mathrm{CN}$ vibration region was measured at 0.89 $\mathrm{cm}^{-1}$. Raman spectra of drop-casted thin-films (glass substrate) were collected using a confocal Raman microscope (Renishaw InVia-Reflex) with an excitation laser at 785 $\mathrm{nm}$, in the edge mode. A 50x objective (Olympus, N.A. 0.5) with a long working distance was used to record the spectra, spanning a range of $100-3000 \mathrm{~cm}^{-1}$. The spectra from each sample smoothed in Origin using adjacent averaging (10 points) and baseline corrected. Absorption spectra were recorded on a Jasco UV-VIS V-730. Emission spectra were recorded on a Jasco FP-8300. Optical micrographs were taken with an Axio Imager 2 (Carl Zeiss Microscopy $\mathrm{GmbH}$, Germany). Crystal structure analysis was accomplished by using a Bruker Apex-II diffractometer with a molybdenum source $\left(\lambda\left(\mathrm{Mo}_{\mathrm{K \alpha}}\right)=0.71073 \AA\right)$ or a STOE Stadivari diffractometer with a copper source $\left[\lambda\left(\mathrm{Cu}_{\mathrm{K \alpha}}\right)=1.54178 \AA\right]$. Data were corrected for sample illumination, air and detector absorption, Lorentz, and polarization effects; ${ }^{[\mathrm{S} 1]}$ absorption by the crystal was treated numerically (Gaussian grid). ${ }^{[S 1-2]}$ The structures were solved by using intrinsic phasing ${ }^{[\mathrm{S} 3]}$ or direct methods with dual-space recycling ${ }^{[\mathrm{S4}]}$ and refined by using full-matrix least-squares methods on F2 against all unique reflections. ${ }^{[S 5]}$ All nonhydrogen atoms were given anisotropic displacement parameters. Hydrogen atoms were input at calculated positions and refined with a riding model. When necessary, disordered groups and/or solvent molecules were subjected to suitable geometry and adp restraints and/or constraints. CCDC 2040320-2040325 contain the supplementary crystallographic data for this paper. These data are provided free of charge by The Cambridge Crystallographic Data Centre (https://www.ccdc.cam.ac.uk/).

\subsection{Synthetic Procedures}

QPP-OMe and PQDC were prepared according to previously reported procedures. ${ }^{[S 6-7]}$

General procedure for the synthesis of $\operatorname{LiTCNQ}$ and $\operatorname{LiF}_{\mathbf{x}} \mathbf{T C N Q}(\mathrm{x}=1,2,4)$ salts: $A$ literature known procedure was applied. ${ }^{[S 8]} \mathbf{T C N Q} / \mathbf{F}_{\mathbf{x}} \mathbf{T C N Q}(95 \mu \mathrm{mol})$ was dissolved in abs. acetonitrile $(1.5 \mathrm{~mL})$ under Ar atmosphere. The solution was cooled to $0{ }^{\circ} \mathrm{C}$ and under stirring a cooled solution of lithium iodide $(19 \mathrm{mg}, 140 \mu \mathrm{mol})$ in abs. acetonitrile $(1.5 \mathrm{~mL})$ was added dropwise. The deep-green mixture was stirred at $0{ }^{\circ} \mathrm{C}$ for $50 \mathrm{~min}$. The formed deep-purple solid was allowed to settle and the solution was decanted. The residue was subsequently washed with acetonitrile $(0.5 \mathrm{~mL})$ and diethyl ether $(4 \times 3$ $\mathrm{mL}$ ) and dried in an Ar-stream to obtain the lithium salts as deep-purple powders (green when dissolved in acetonitrile).

LiTCNQ. IR (ATR): $\tilde{v}\left[\mathrm{~cm}^{-1}\right]=2205$ (s), 2192 (s), 2187 (s), 2176 (s), 2143 (m), 1576 (s), $1574(\mathrm{~s}), 1570(\mathrm{~s}), 1560(\mathrm{~m}), 1555(\mathrm{~m}), 1506(\mathrm{~s}), 1497(\mathrm{~m}), 1481(\mathrm{~m}), 1359(\mathrm{~s}), 1319$ (vs), 1244 (w), 1232 (w), 1180 (vs), 1176 (vs), 1169 (s), $989(\mathrm{~m}), 958(\mathrm{~m}), 952(\mathrm{~m}), 887$ (w), 824 (vs), $809(\mathrm{~m}), 722(\mathrm{~m}), 621$ (w), $619(\mathrm{w})$. UV/Vis (acetonitrile): $\lambda_{\mathrm{abs}}[\mathrm{nm}]=842$, 823, 760, 743, 680, 665, 420, 407. 
LiFTCNQ. IR (ATR): $\tilde{v}$ [cm-1] = 2220 (s), 2209 (vs), 2190 (vs), 1596 (s), 1510 (s), 1494 (s), 1361 (vs), 1327 (s), $1262(\mathrm{~m}), 1216(\mathrm{~m}), 1190(\mathrm{~s}), 1154(\mathrm{w}), 1119(\mathrm{w}), 1025(\mathrm{w})$, $988(\mathrm{vw}), 930(\mathrm{vw}), 907(\mathrm{vw}), 870(\mathrm{~m}), 852(\mathrm{~m}), 800(\mathrm{~m}), 711(\mathrm{vw}), 683(\mathrm{w}), 644(\mathrm{w})$, $628(\mathrm{w})$. UV/Vis (acetonitrile): $\lambda_{\mathrm{abs}}[\mathrm{nm}]=845,760,744,680,418$.

LiF $_{2}$ TCNQ. IR (ATR): $\tilde{v}\left[\mathrm{~cm}^{-1}\right]=2214$ (vs), 2192 (vs), $1612(\mathrm{~m}), 1531(\mathrm{~m}), 1517$ (s), 1487 (s), 1372 (vs), $1344(\mathrm{~s}), 1302(\mathrm{~m}), 1249(\mathrm{~m}), 1213(\mathrm{~s}), 1189(\mathrm{~s}), 1164(\mathrm{~m}), 1159$ $(\mathrm{m}), 1136(\mathrm{w}), 1029(\mathrm{w}), 935(\mathrm{vw}), 916(\mathrm{vw}), 862(\mathrm{~s}), 790(\mathrm{~s}), 687(\mathrm{w}), 641(\mathrm{~m})$. UV/Vis (acetonitrile): $\lambda_{\text {abs }}[\mathrm{nm}]=843,759,743,678,424,366$.

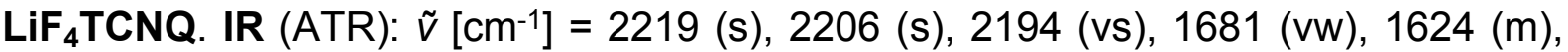
1530 (s), 1498 (s), $1448(\mathrm{w}), 1365$ (s), 1351 (vs), $1269(\mathrm{~m}), 1216(\mathrm{~m}), 1203$ (s), 1154 (w), $1059(\mathrm{vw}), 1039(\mathrm{vw}), 972(\mathrm{vs}), 907(\mathrm{vw}), 878(\mathrm{w}), 796(\mathrm{~m}), 720(\mathrm{vw}), 658(\mathrm{vw})$, $642(\mathrm{w})$. UV/Vis (acetonitrile): $\lambda_{\text {abs }}[\mathrm{nm}]=855,753,687,411$.

\subsection{Sample preparation of Charge Transfer Complexes}

General procedure for single crystal growth suitable for SCXRD: $150 \mu \mathrm{L}$ of a freshly filtered solution of QPP-OMe $\left(0.3 \mathrm{mM}, \mathrm{CHCl}_{3}\right)$ was mixed with $150 \mu \mathrm{L}$ of a freshly filtered acceptor solution $\left(0.3 \mathrm{mM}, \mathrm{CHCl}_{3}\right)$ in an open $\mathrm{HPLC}$ vial. The vial was then placed in a larger, closed vial containing $1 \mathrm{~mL} \mathrm{MeOH}$ and left at constant r.t. for several days without moving.

General procedure for thin-film fabrication: A freshly filtered solution of QPP-OMe (2 $\mathrm{mM}, \mathrm{CHCl}_{3}$ ) was mixed with a freshly filtered acceptor solution $\left(2 \mathrm{mM}, \mathrm{CHCl}_{3}\right)$ in a 1:1 ratio. $100 \mu \mathrm{L}$ of this mixture was then drop-casted onto a glass substrate $(1 \times 1 \mathrm{~cm})$. After the solvent had evaporated at rt, the films were annealed at $60^{\circ} \mathrm{C}$ for $2 \mathrm{~min}$. The color of the films corresponded to the color of the single crystals indicating complex formation.

General procedure for preparation of IR samples: $150 \mu \mathrm{L}$ of a freshly filtered solution of QPP-OMe $\left(0.3 \mathrm{mM}, \mathrm{CHCl}_{3}\right)$ was mixed with $150 \mu \mathrm{L}$ of a freshly filtered acceptor solution $\left(0.3 \mathrm{mM}, \mathrm{CHCl}_{3}\right)$ and the solvent was evaporated at rt. The residual amorphous powder was used for IR analysis. The color of the powders corresponded to the color of the single crystals indicating complex formation.

QPP-OMe/DNT. IR (ATR): $\tilde{v}\left[\mathrm{~cm}^{-1}\right]=2959(\mathrm{~m}), 2920(\mathrm{~s}), 2851(\mathrm{~m}), 1730(\mathrm{~m}), 1718$ $(\mathrm{m}), 1701(\mathrm{w}), 1684(\mathrm{~m}), 1666(\mathrm{~m}), 1653(\mathrm{~m}), 1634(\mathrm{~m}), 1608(\mathrm{~m}), 1556(\mathrm{w}), 1535(\mathrm{~s})$, $1460(\mathrm{~m}), 1418(\mathrm{~m}), 1394(\mathrm{w}), 1365(\mathrm{~m}), 1342(\mathrm{~s}), 1281(\mathrm{~m}), 1261(\mathrm{~s}), 1227(\mathrm{~m}), 1203$ $(\mathrm{m}), 1175(\mathrm{w}), 1155(\mathrm{~m}), 1090$ (vs), 1020 (vs), 903 (w), 889 (w), 864 (w), 800 (vs), 768 $(w), 739(m), 702(w), 685(w), 669(w), 650(w), 629(w), 606(w)$.

QPP-OMe/TCNQ. IR (ATR): $\tilde{v}\left[\mathrm{~cm}^{-1}\right]=2955(\mathrm{~m}), 2924(\mathrm{~m}), 2854(\mathrm{w}), 2222(\mathrm{w}), 1736$ (vw), $1689(\mathrm{w}), 1612(\mathrm{w}), 1574(\mathrm{vw}), 1543(\mathrm{w}), 1495(\mathrm{w}), 1479(\mathrm{w}), 1460(\mathrm{~m}), 1445(\mathrm{~m})$, $1421(\mathrm{w}), 1394(\mathrm{w}), 1364(\mathrm{~m}), 1329(\mathrm{~m}), 1315(\mathrm{~m}), 1281(\mathrm{~m}), 1271(\mathrm{~m}), 1242(\mathrm{~m}), 1227$ (w), $1205(\mathrm{w}), 1184(\mathrm{w}), 1171(\mathrm{w}), 1153(\mathrm{~m}), 1144(\mathrm{~m}), 1086(\mathrm{~m}), 1022(\mathrm{~m}), 974(\mathrm{vw})$, $904(\mathrm{~m}), 889(\mathrm{w}), 877(\mathrm{w}), 862(\mathrm{w}), 841(\mathrm{~s}), 804(\mathrm{~m}), 775(\mathrm{w}), 766(\mathrm{~m}), 742(\mathrm{vs}), 712$ $(w), 656(w), 644(w), 629(m), 615(w), 604(w)$.

QPP-OMe/FTCNQ. IR (ATR): $\tilde{v}\left[\mathrm{~cm}^{-1}\right]=2957(\mathrm{~m}), 2924(\mathrm{~m}), 2854(\mathrm{w}), 2222(\mathrm{w}), 1684$ $(w), 1632(w), 1614(m), 1551(w), 1497(w), 1479(w), 1460(m), 1445(m), 1423(w)$, $1394(\mathrm{w}), 1364(\mathrm{~m}), 1329(\mathrm{~m}), 1315(\mathrm{~m}), 1281(\mathrm{~m}), 1271(\mathrm{~m}), 1242(\mathrm{~m}), 1227(\mathrm{~m}), 1207$ $(\mathrm{m}), 1171(\mathrm{~m}), 1155(\mathrm{~m}), 1140(\mathrm{~m}), 1097(\mathrm{~m}), 1086(\mathrm{~m}), 1070(\mathrm{~m}), 1022(\mathrm{~m}), 980(\mathrm{w})$, 
935 (w), $903(\mathrm{~m}), 890(\mathrm{~m}), 874(\mathrm{~m}), 864(\mathrm{~m}), 806(\mathrm{~s}), 766(\mathrm{~m}), 741(\mathrm{vs}), 712(\mathrm{~m}), 658$ $(\mathrm{m}), 646(\mathrm{~m}), 629(\mathrm{~m}), 613(\mathrm{~m})$.

QPP-OMe/F ${ }_{2}$ TCNQ. IR (ATR): $\tilde{v}$ [cm-1] = 3061 (vw), $3043(\mathrm{vw}), 3005(\mathrm{vw}), 2955(\mathrm{~m})$, $2924(\mathrm{~m}), 2854(\mathrm{w}), 2222(\mathrm{vw}), 1688(\mathrm{vw}), 1612(\mathrm{~m}), 1574(\mathrm{~m}), 1545(\mathrm{w}), 1495(\mathrm{w})$, $1479(\mathrm{w}), 1460(\mathrm{~s}), 1445(\mathrm{~m}), 1423(\mathrm{w}), 1389(\mathrm{~m}), 1364(\mathrm{~m}), 1329(\mathrm{~m}), 1315(\mathrm{~m}), 1281$ $(\mathrm{m}), 1271(\mathrm{~m}), 1242(\mathrm{~m}), 1227(\mathrm{~m}), 1208(\mathrm{~m}), 1171(\mathrm{~m}), 1155(\mathrm{~m}), 1138(\mathrm{~m}), 1097(\mathrm{~m})$, $1086(\mathrm{~m}), 1070(\mathrm{w}), 1024(\mathrm{~m}), 978(\mathrm{vw}), 933(\mathrm{vw}), 903(\mathrm{~m}), 887(\mathrm{~m}), 874(\mathrm{w}), 862(\mathrm{w})$, $804(\mathrm{~m}), 766(\mathrm{~m}), 741(\mathrm{vs}), 712(\mathrm{w}), 673(\mathrm{vw}), 658(\mathrm{w}), 644(\mathrm{w}), 629(\mathrm{~m}), 615(\mathrm{w})$.

PP-OMe/F ${ }_{4}$ TCNQ. IR (ATR): $\tilde{v}$ [ $\left.\mathrm{cm}^{-1}\right]=2957(\mathrm{w}), 2924(\mathrm{~m}), 2854(\mathrm{w}), 1684(\mathrm{vw}), 1653$ (vw), 1634 (vw), $1614(\mathrm{w}), 1597(\mathrm{w}), 1589(\mathrm{w}), 1558(\mathrm{vw}), 1541$ (vw), $1495(\mathrm{w}), 1479$ $(\mathrm{w}), 1460(\mathrm{~m}), 1443(\mathrm{~m}), 1421(\mathrm{w}), 1394(\mathrm{w}), 1364(\mathrm{~m}), 1342(\mathrm{~m}), 1329(\mathrm{~m}), 1315(\mathrm{w})$, $1271(\mathrm{~m}), 1242(\mathrm{~m}), 1227(\mathrm{w}), 1203(\mathrm{w}), 1171(\mathrm{w}), 1155(\mathrm{~m}), 1136(\mathrm{~m}), 1097(\mathrm{~m}), 1086$ (m), $1055(w), 1024(m), 974(m), 931(v w), 903(w), 887(w), 877(w), 862(w), 804$ (m), 739 (vs), $712(w), 679(w), 656(w), 646(w), 620(m), 604(w)$.

PP-OMe/PQDC. IR (ATR): $\tilde{v}$ [cm-1] = $3362(\mathrm{vw}), 3310(\mathrm{w}), 3184(\mathrm{vw}), 2955$ (m), 2920 (vs), 2851 (s), 1734 (w), $1718(\mathrm{w}), 1701(\mathrm{w}), 1655(\mathrm{~m}), 1632(\mathrm{~m}), 1608(\mathrm{w}), 1556(\mathrm{vw})$, $1539(\mathrm{w}), 1460(\mathrm{~m}), 1419(\mathrm{w}), 1364(\mathrm{~m}), 1325(\mathrm{w}), 1281(\mathrm{w}), 1246(\mathrm{w}), 1225(\mathrm{w}), 1203$ $(w), 1175(w), 1155(\mathrm{~m}), 1103(\mathrm{~m}), 1088(\mathrm{~m}), 1024(\mathrm{w}), 972(\mathrm{vw}), 901(\mathrm{w}), 889(\mathrm{w}), 864$ $(\mathrm{vw}), 835(\mathrm{~m}), 800(\mathrm{w}), 768(\mathrm{w}), 741(\mathrm{~m}), 717(\mathrm{~m}), 648(\mathrm{w}), 629(\mathrm{w}), 607(\mathrm{w})$.

General procedure for the preparation of samples used for UV/Vis spectroscopy in $\mathrm{MeOH}$ solution: $2 \mathrm{~mL}$ of a freshly filtered solution of QPP-OMe $\left(5 \mathrm{mM}, \mathrm{CHCl}_{3}\right)$ was mixed with $2 \mathrm{~mL}$ of a freshly filtered acceptor solution $\left(2 \mathrm{mM}, \mathrm{CHCl}_{3}\right)$ and the solvent was reduced almost to a volume of approx. $0.5 \mathrm{~mL}$. The suspension was centrifuged and the mother liquor was removed. The residue was washed with $\mathrm{MeOH}(100 \mu \mathrm{L})$ under ultrasonication (1 $\mathrm{min}$.) and the solvent removed via centrifugation. The color of the remaining solid corresponded to the color of the single crystals indicating complex formation. The residue was dissolved in $\mathrm{MeOH}(0.5 \mathrm{~mL})$ under ultrasonication and gentle heating $\left(50^{\circ} \mathrm{C}\right)$ and used for absorption spectroscopy after filtration. The color of the powders corresponded to the color of the single crystals indicating complex formation.

QPP-OMe/DNT. UV/Vis (MeOH): $\lambda_{\mathrm{abs}}[\mathrm{nm}]=417,394,332$.

QPP-OMe/TCNQ. UV/Vis $(\mathrm{MeOH}): \lambda_{\mathrm{abs}}[\mathrm{nm}]=837,740,677,416,394,333,277$.

QPP-OMe/FTCNQ. UV/Vis (MeOH): $\lambda_{\text {abs }}[\mathrm{nm}]=841,742,677,417,394$, 333, 277.

QPP-OMe/F ${ }_{2}$ TCNQ. UV/Vis $(\mathrm{MeOH}): \lambda_{\mathrm{abs}}[\mathrm{nm}]=841,741,677,421,350,280$.

QPP-OMe/F 4 TCNQ. UV/Vis $(\mathrm{MeOH}): \lambda_{\mathrm{abs}}[\mathrm{nm}]=854,753,394,333,277$.

QPP-OMe/PQDC. UV/Vis $(\mathrm{MeOH}): \lambda_{\mathrm{abs}}[\mathrm{nm}]=418,340,310,280$. 


\section{Optical Characterization of Thin-Films}
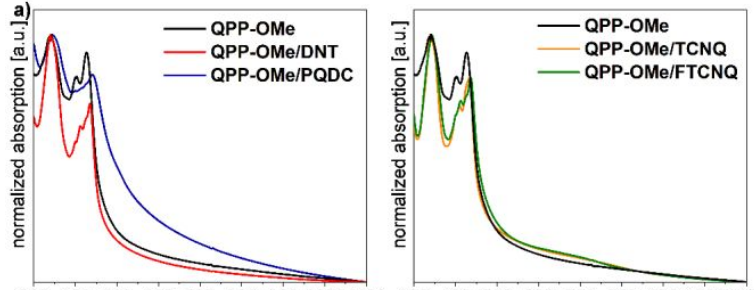

3004005006007008009001000110030040050060070080090010001100
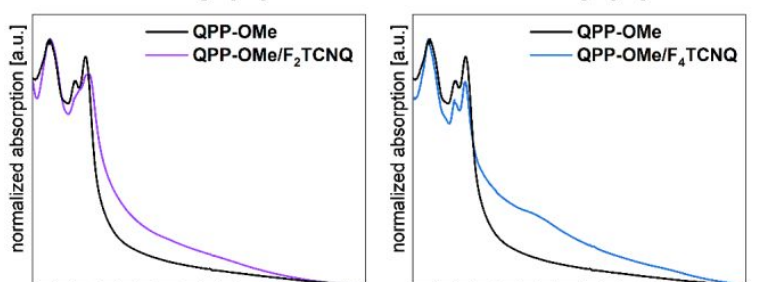

3004005006007008009001000110030040050060070080090010001100

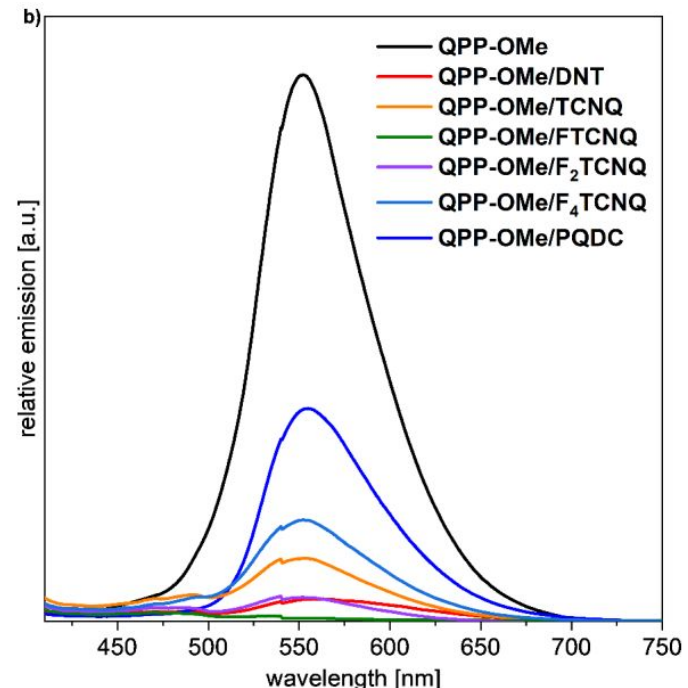

Figure S1. a) Normalized UV/Vis spectra of drop-casted thin-films $\left(\mathrm{CHCl}_{3}\right)$ of QPP-OMe and 1:1mixtures of QPP-OMe with DNT, TCNQ, $\mathbf{F}_{\mathbf{x}} \mathbf{T C N Q}(\mathrm{x}=1,2,4)$ and PQDC. b) Corresponding emission spectra $\left(\lambda_{\mathrm{ex}}=385 \mathrm{~nm}\right)$. 

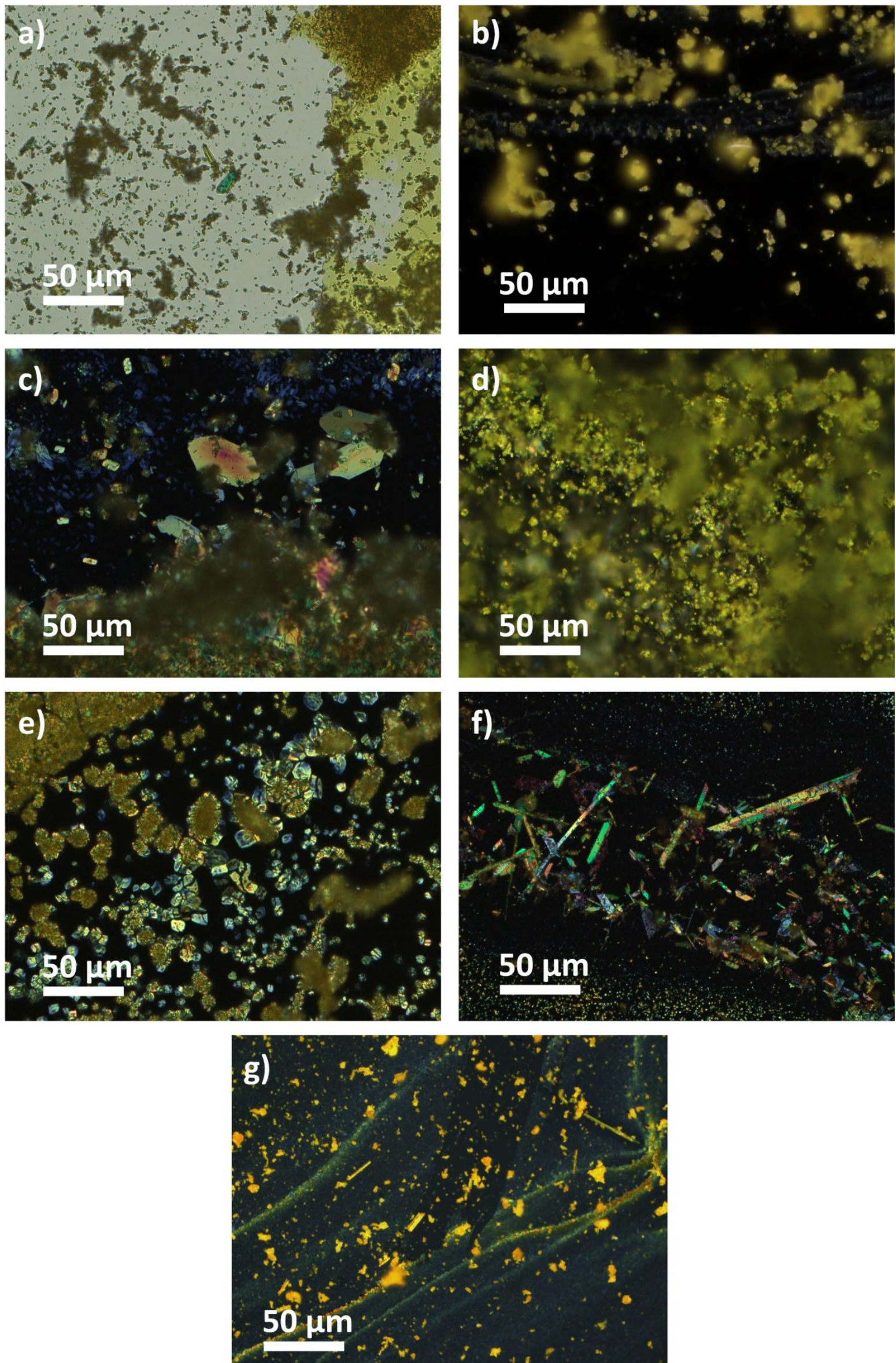

Figure S2. Optical micrographs (transmission mode) of drop-casted thin-films $\left(\mathrm{CHCl}_{3}\right)$ of QPP-OMe (a) and 1:1-mixtures of QPP-OMe with DNT (b), TCNQ (c), $\mathbf{F}_{\mathbf{x}}$ TCNQ $(\mathrm{x}=1,2,4)$ (d-f) and PQDC(g). 


\section{Vibrational Spectra}

\subsection{FT-IR Spectra}

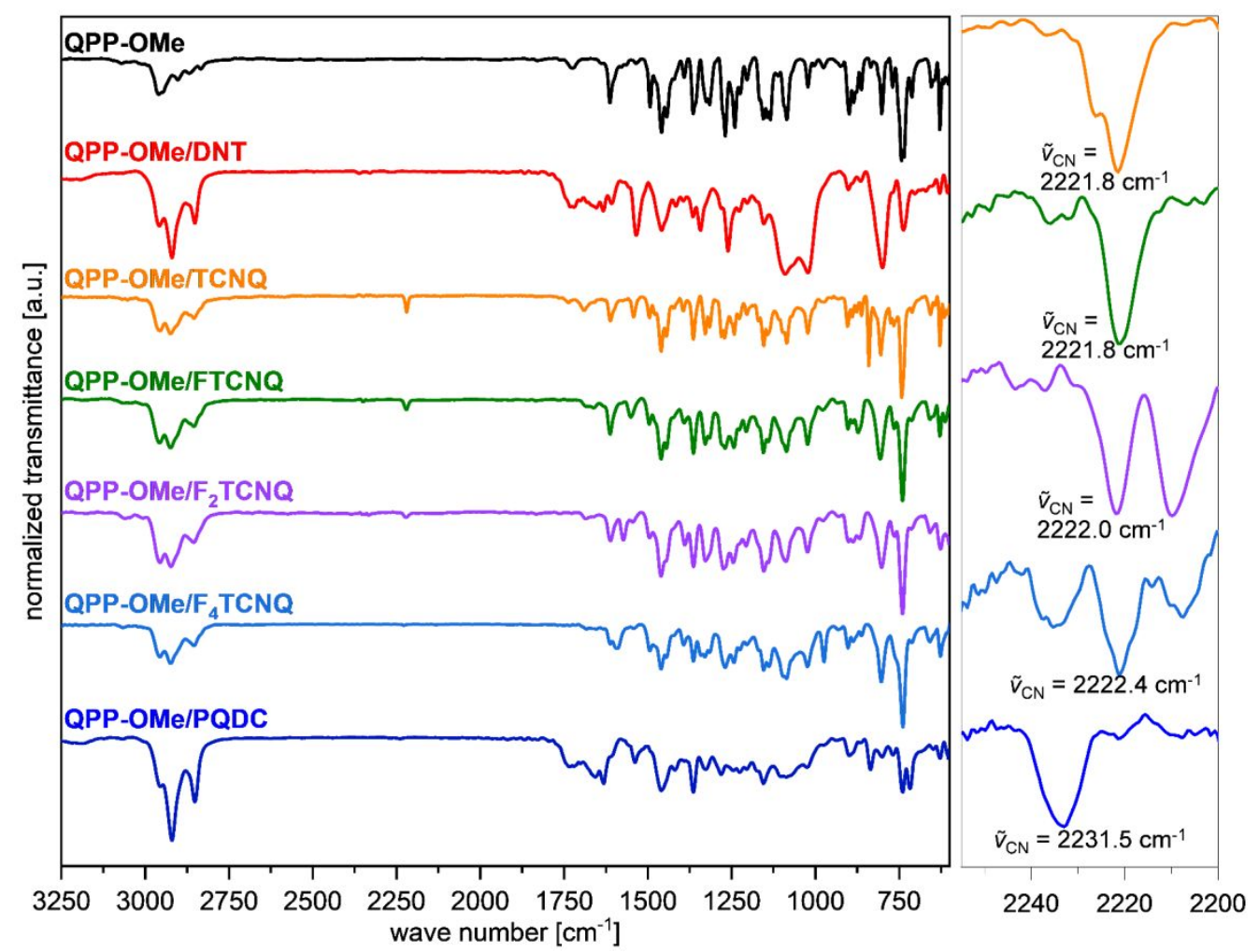

Figure S3. Baseline-corrected FT-IR spectra (ATR) of powdered QPP-OMe and 1:1 mixtures of QPPOMe with DNT, TCNQ, $\mathbf{F}_{\mathbf{x}} \mathrm{TCNQ}(\mathrm{x}=1,2,4)$ and PQDC. Right: Enlarged CN vibration region. The spectra were smoothed using adjacent averaging (5-12 points) in Origin.
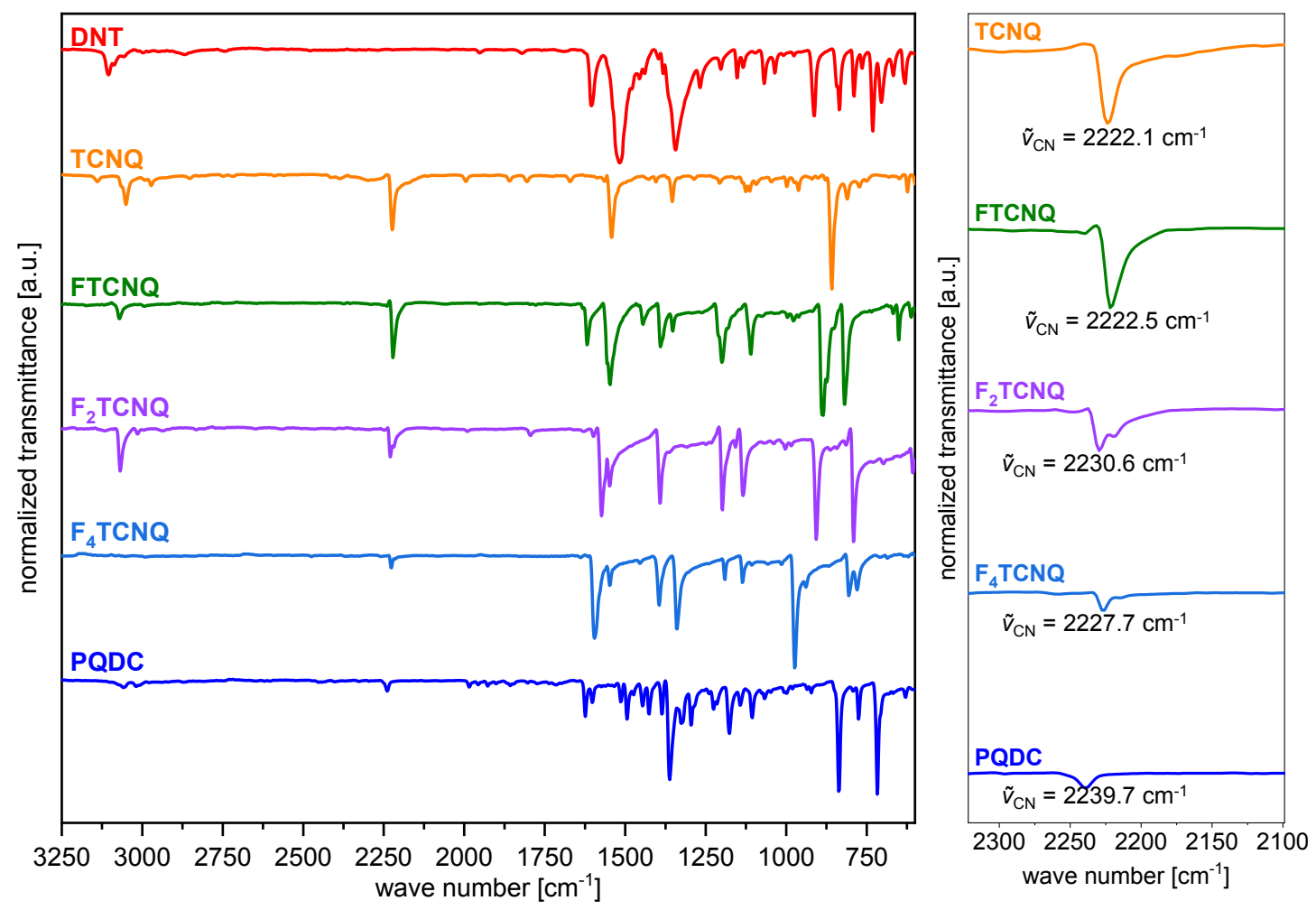

Figure S4. Baseline-corrected FT-IR spectra (ATR) of powdered DNT, TCNQ, $\mathbf{F}_{\mathbf{x}} \mathbf{T C N Q}(\mathrm{x}=1,2,4)$ and PQDC. Right: Enlarged CN vibration region. 


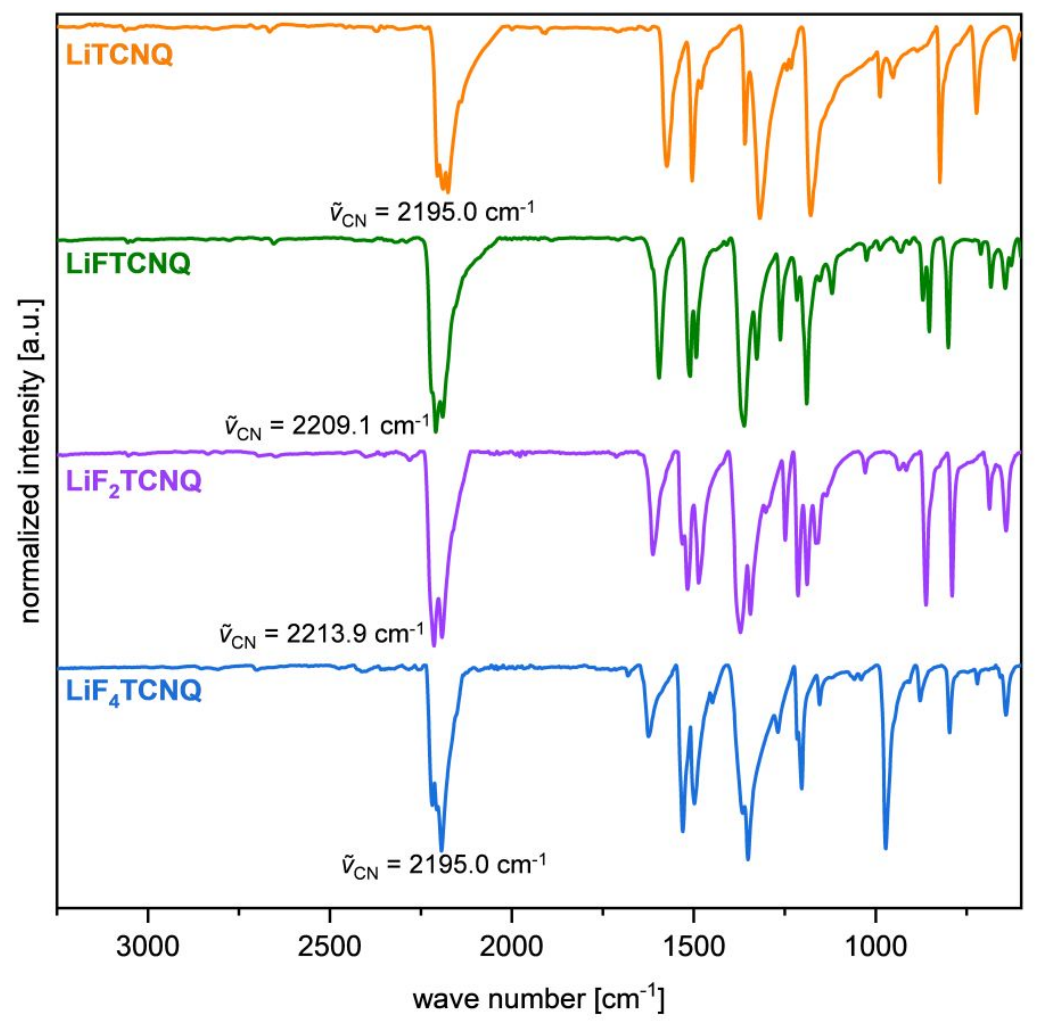

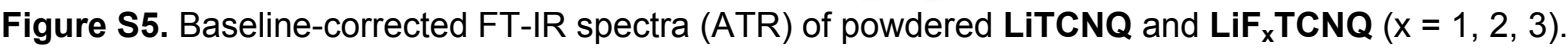

\subsection{Raman Spectra}
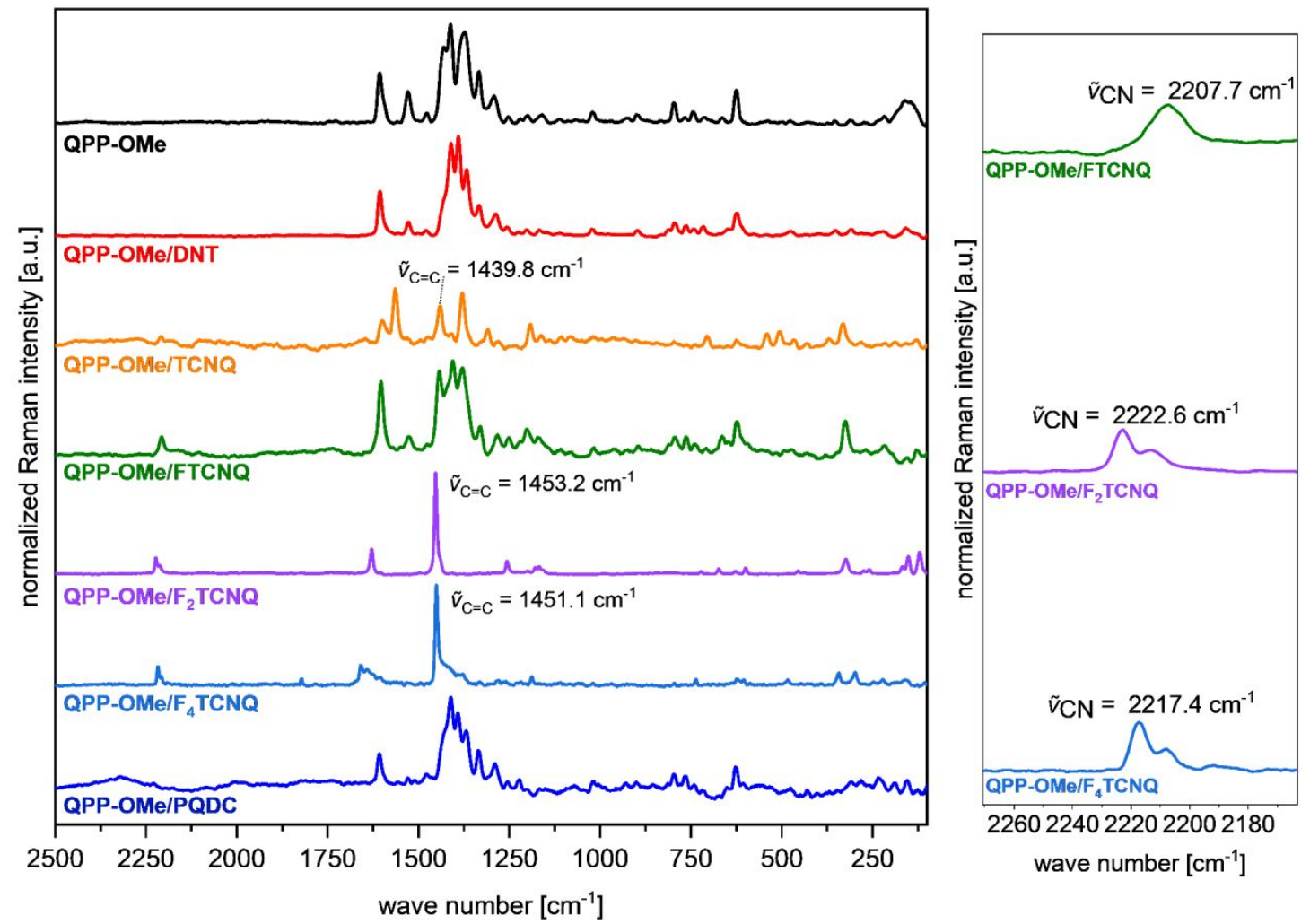

Figure S6. Baseline-corrected Raman spectra $\left(\lambda_{\text {ex }}=785 \mathrm{~nm}\right)$ of drop-casted thin-films $\left(\mathrm{CHCl}_{3}\right)$ of QPPOMe and 1:1 mixtures of QPP-OMe with DNT, TCNQ, $\mathbf{F}_{\mathbf{x}} \mathbf{T C N Q}(\mathrm{x}=1,2,4)$ and PQDC. The spectra were smoothed using adjacent averaging (5-10 points) in Spartan. Right: Enlarged CN vibration region. 

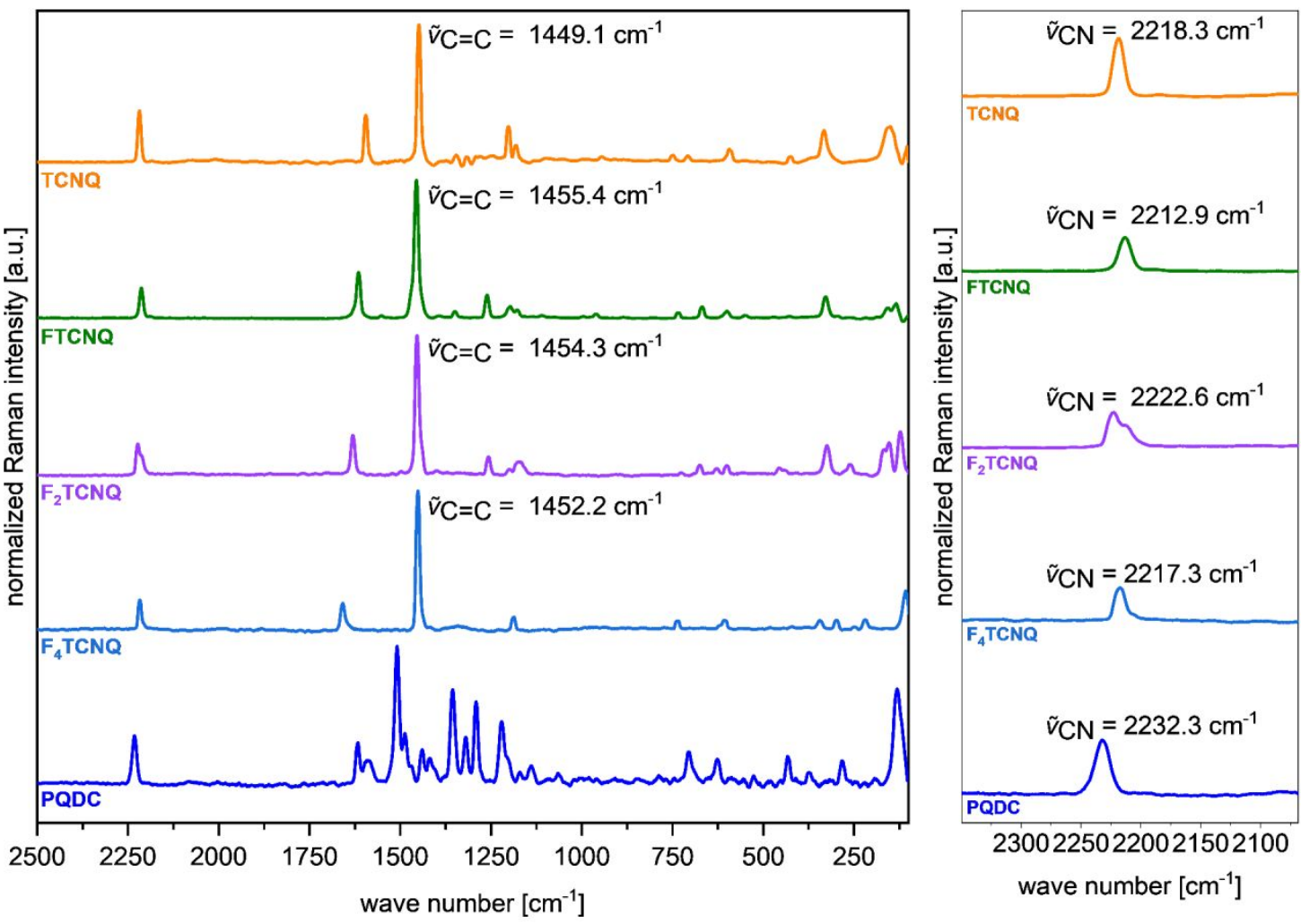

Figure S7. Baseline-corrected Raman spectra $\left(\lambda_{\mathrm{ex}}=785 \mathrm{~nm}\right)$ of drop-casted thin-films $\left(\mathrm{CHCl}_{3}\right)$ of TCNQ, $F_{\mathbf{x}} \mathbf{T C N Q}(\mathbf{x}=1,2,4)$ and PQDC. The spectra were smoothed using adjacent averaging (5-10 points) in Spartan. Right: Enlarged CN vibration region.

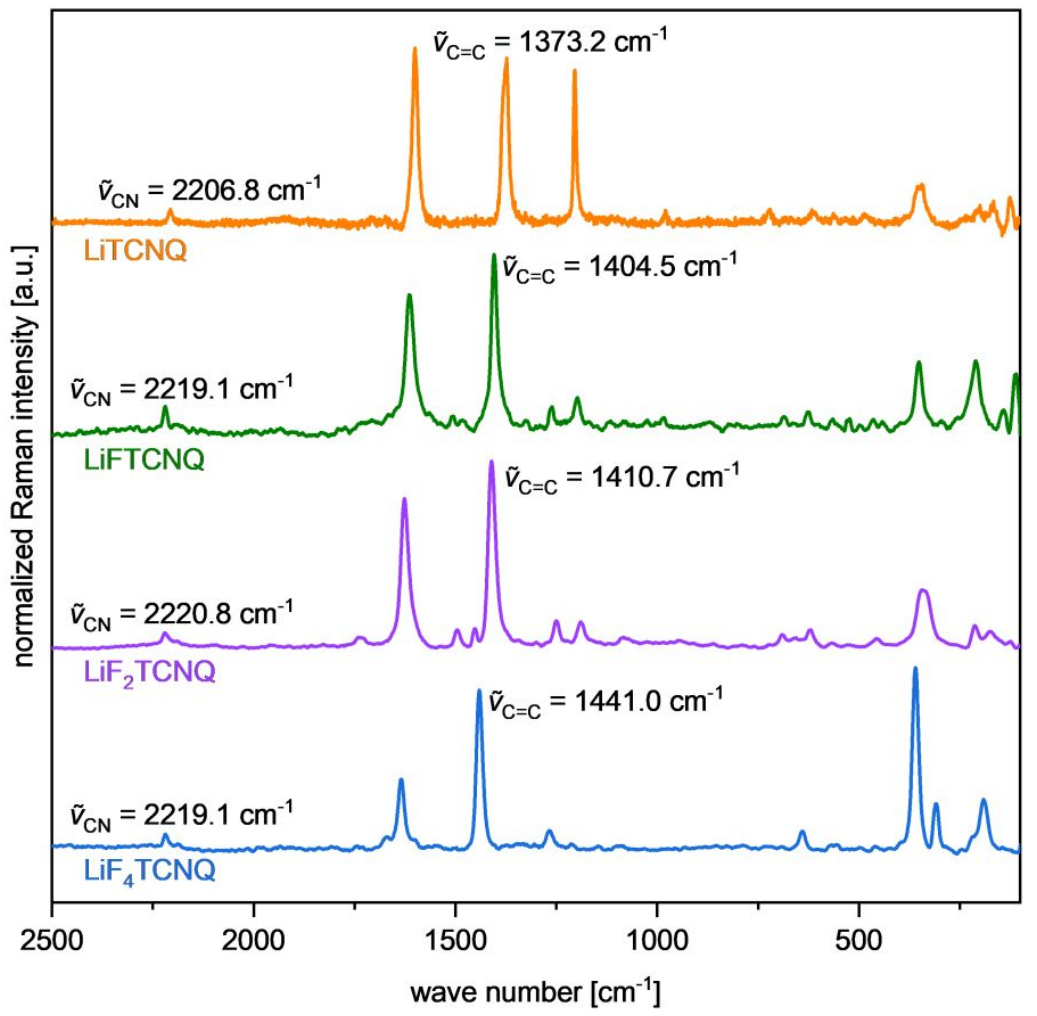

Figure S8. Baseline-corrected Raman spectra $\left(\lambda_{\mathrm{ex}}=785 \mathrm{~nm}\right)$ of drop-casted thin-films (acetonitrile) of $\operatorname{LiF}_{\mathbf{x}} \mathbf{T C N Q}(\mathrm{x}=1,2,4)$. The spectra were smoothed using adjacent averaging (5-10 points) in Spartan. 


\section{$4 \quad$ UV/Vis Spectra}
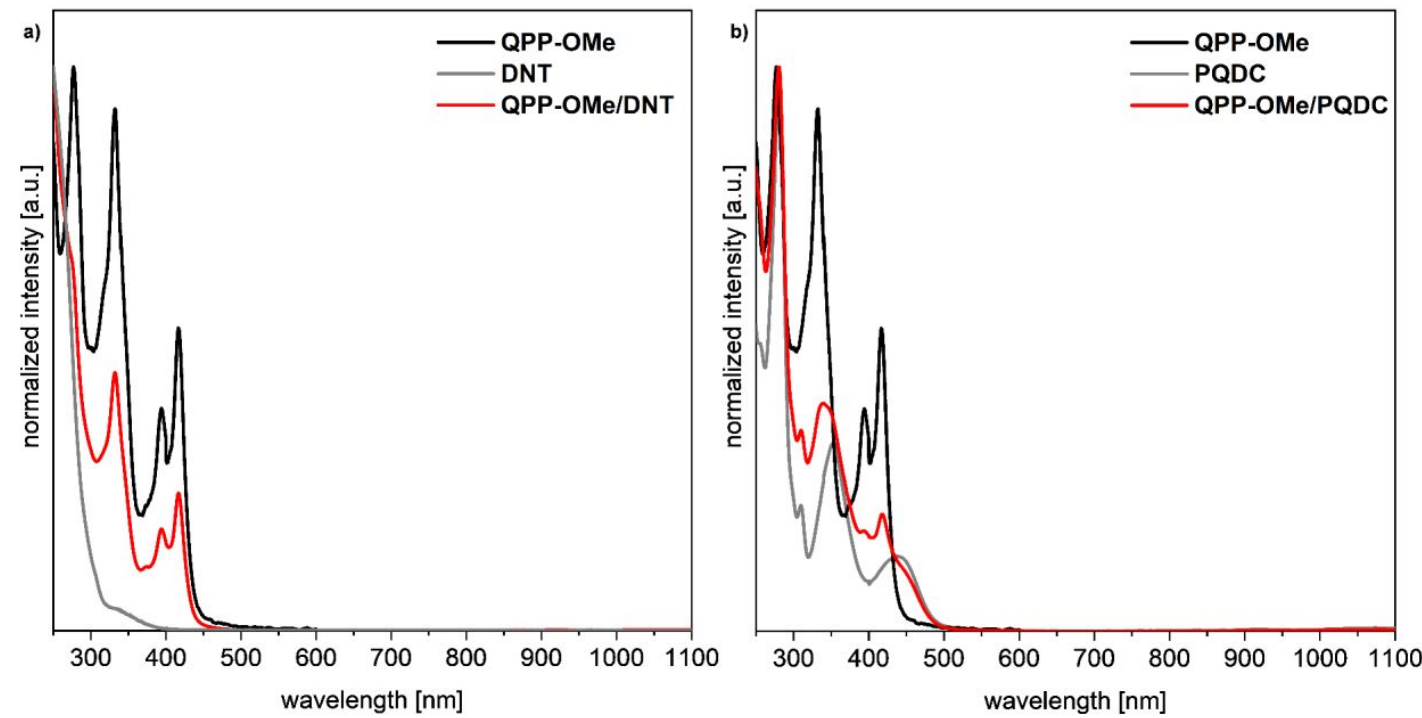

Figure S9. Normalized UV/Vis spectra of a) QPP-OMe/DNT and b QPP-OMe/PQDC in MeOH at rt.
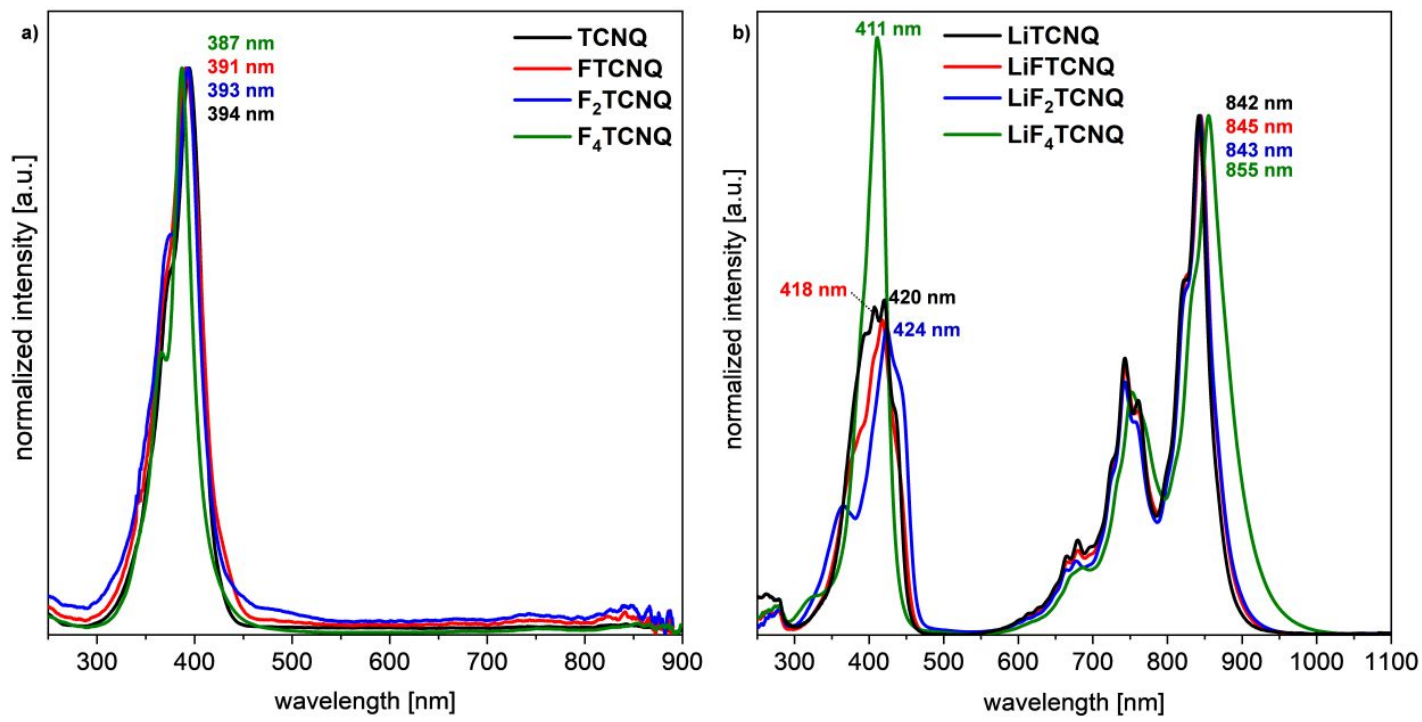

Figure S10. Normalized UV/Vis spectra of a) TCNQ and $\mathbf{F}_{x} \mathbf{T C N Q}(x=1,2,4)$ in acetonitrile at $r$ and $\left.b\right)$ $\operatorname{LiF}_{\mathbf{x}} \mathbf{T C N Q}(\mathrm{x}=1,2,4)$ in acetonitrile at rt.

\section{Crystal Structure Analysis}

\subsection{Determination of Distances and Displacement of $\pi$ Stacked Planes}

$\pi$-T-distances $d_{\pi, \mathrm{D}-\mathrm{D}}$ of adjacent QPP molecules and interplanar distances $d_{\mathrm{D}-\mathrm{D}}$ were calculated from the distance of two planes containing the inner carbon atoms of the aromatic backbone (depicted as balls in Fig. S11a) in Mercury. Donor-acceptor distances were measured from a centroid generated from the acceptor backbone to the QPP plane.

The displacement (offset) of two $\pi$ stacked QPP molecules along the molecular long axis was calculated from the distance of their centroids $d_{\text {cent }}$ and $d_{\pi, \mathrm{D}-\mathrm{D}}$ (Pythagoras, Fig. S11b). For determination of the center, centroids were generated in Mercury using the carbon atoms of the aromatic backbone (depicted as balls in Fig. S11a). For $d_{x^{\prime}}$ the 
centroid generated from the two aromatic carbons were used as described above (Fig. S11 c,d).

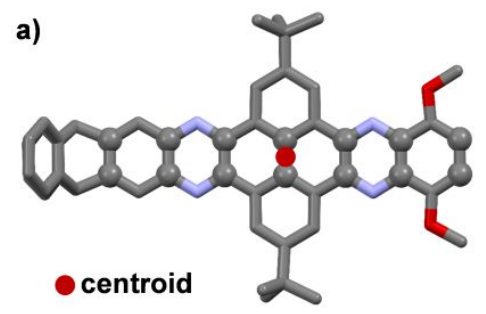

b)
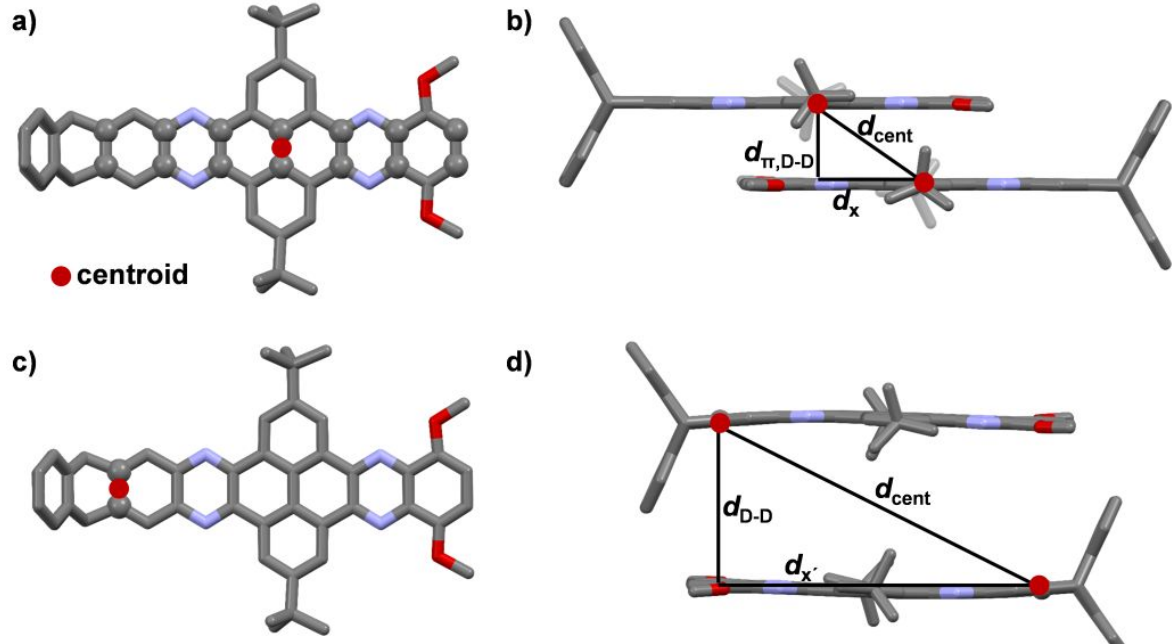

d)

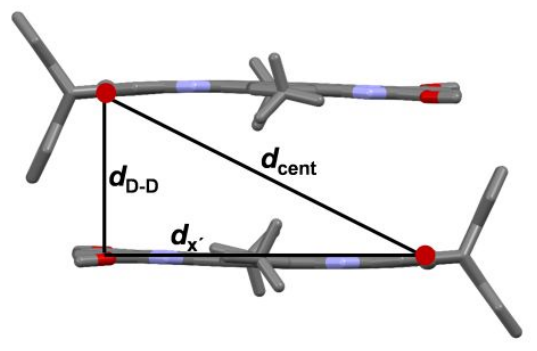

Figure S11. Determination of donor-donor $\pi-$ and interplanar distances and offsets: a) Carbon atoms depicted as balls were used to generate planes to calculate $\pi-\pi-$ and interplanar distances $\left(d_{\pi, \mathrm{D}-\mathrm{D}}\right.$ and $d_{\mathrm{D}-\mathrm{D}}$ ) and centroids to determine the offset $d_{\mathrm{x}}$ along the molecular long axis of two $\pi$ stacked QPPs (b) .c,d) Atoms used to generate centroids to calculate $d_{x}$.

\subsection{Crystal Data}

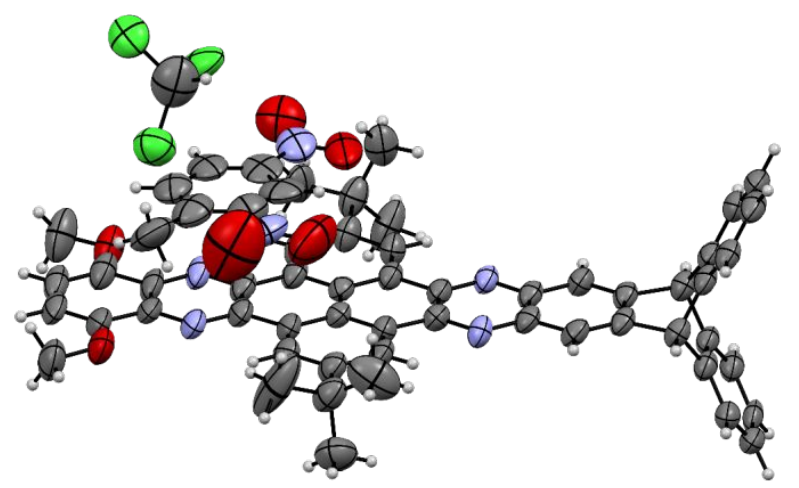

Figure S12. Thermal atomic displacement ellipsoid plot of the asymmetric unit of QPP-OMe/DNT. The ellipsoids of non-hydrogen atoms are drawn at the $50 \%$ probability level and hydrogen atoms are represented by a sphere of arbitrary size.

Table S1. Crystal data and structure refinement for QPP-OMe/DNT.

CCDC
Solvent
Empirical formula
Formula weight
Temperature
Wavelength
Crystal system
Space group
Z
Unit cell dimensions

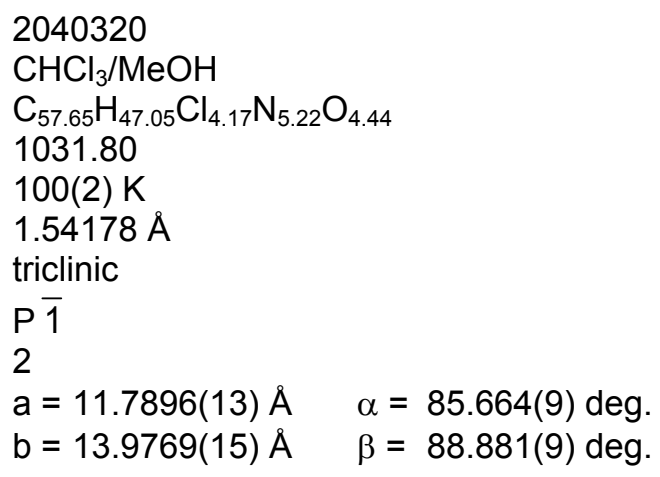


Volume

Density (calculated)

Absorption coefficient

Crystal shape

Crystal size

Crystal colour

Theta range for data collection

Index ranges

Reflections collected

Independent reflections

Observed reflections

Absorption correction

Max. and min. transmission

Refinement method

Data/restraints/parameters

Goodness-of-fit on $\mathrm{F}^{2}$

Final $\mathrm{R}$ indices ( $\mathrm{I}>2$ sigma(I))

Largest diff. peak and hole

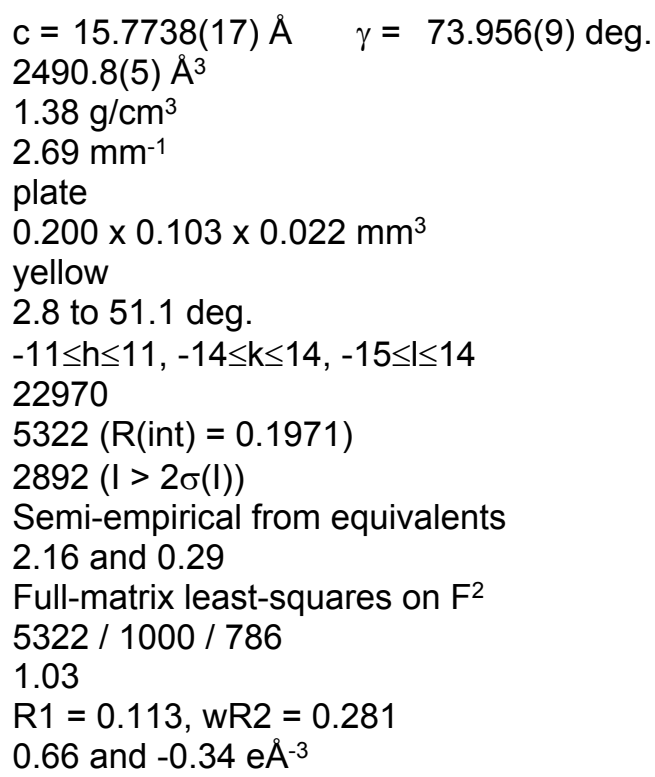

Table S2. Atomic coordinates and equivalent isotropic displacement parameters $\left(\AA^{2}\right)$ for QPP-OMe/DNT. $U_{e q}$ is defined as one third of the trace of the orthogonalized $U_{i j}$ tensor.

\begin{tabular}{lllll} 
Atom & $\mathrm{x}$ & $\mathrm{y}$ & $\mathrm{z}$ & $\mathrm{U}$ \\
& & & & \\
\hline & & & & \\
C11 & $-0.0755(9)$ & $0.6124(8)$ & $0.2279(6)$ & $0.079(3)$ \\
C12 & $-0.0203(8)$ & $0.6035(7)$ & $0.3103(5)$ & $0.067(2)$ \\
N13 & $0.0611(7)$ & $0.5167(5)$ & $0.3347(4)$ & $0.069(2)$ \\
C14 & $0.1088(8)$ & $0.5111(6)$ & $0.4125(5)$ & $0.060(2)$ \\
C15 & $0.1978(8)$ & $0.4172(6)$ & $0.4408(5)$ & $0.060(2)$ \\
C16 & $0.2318(9)$ & $0.3390(7)$ & $0.3916(6)$ & $0.071(3)$ \\
H16 & 0.1954 & 0.3440 & 0.3376 & 0.085 \\
C17 & $0.3193(8)$ & $0.2503(6)$ & $0.4172(5)$ & $0.069(2)$ \\
C18 & $0.3670(8)$ & $0.2451(7)$ & $0.4978(5)$ & $0.061(2)$ \\
H18 & 0.4240 & 0.1856 & 0.5179 & 0.074 \\
C19 & $0.3343(7)$ & $0.3236(6)$ & $0.5499(5)$ & $0.052(2)$ \\
C20 & $0.3872(7)$ & $0.3164(6)$ & $0.6351(5)$ & $0.053(2)$ \\
N21 & $0.4677(6)$ & $0.2299(5)$ & $0.6601(4)$ & $0.0531(18)$ \\
C22 & $0.5149(7)$ & $0.2252(6)$ & $0.7382(5)$ & $0.052(2)$ \\
C23 & $0.6017(7)$ & $0.1369(6)$ & $0.7672(5)$ & $0.053(2)$ \\
H23 & 0.6245 & 0.0822 & 0.7324 & 0.064 \\
C24 & $0.6516(7)$ & $0.1309(6)$ & $0.8444(5)$ & $0.051(2)$ \\
C25 & $0.7452(7)$ & $0.0406(6)$ & $0.8868(5)$ & $0.054(2)$ \\
H25 & 0.7680 & -0.0179 & 0.8511 & 0.065 \\
C26 & $0.6903(7)$ & $0.0187(6)$ & $0.9705(5)$ & $0.052(2)$ \\
C27 & $0.6688(7)$ & $-0.0718(6)$ & $0.9995(5)$ & $0.058(2)$ \\
H27 & 0.6890 & -0.1268 & 0.9650 & 0.069 \\
C28 & $0.6187(7)$ & $-0.0812(7)$ & $1.0777(5)$ & $0.058(2)$ \\
H28 & 0.6025 & -0.1422 & 1.0965 & 0.070 \\
C29 & $0.5913(7)$ & $-0.0021(7)$ & $1.1298(5)$ & $0.062(2)$ \\
H29 & 0.5596 & -0.0103 & 1.1848 & 0.074 \\
C30 & $0.6100(7)$ & $0.0885(7)$ & $1.1019(5)$ & $0.059(2)$ \\
H30 & 0.5888 & 0.1432 & 1.1367 & 0.071 \\
C31 & $0.6599(7)$ & $0.0986(6)$ & $1.0224(5)$ & $0.054(2)$ \\
C32 & $0.8479(7)$ & $0.0799(6)$ & $0.9080(5)$ & $0.053(2)$ \\
C33 & $0.9644(7)$ & $0.0417(6)$ & $0.8827(5)$ & $0.059(2)$ \\
H33 & 0.9858 & -0.0146 & 0.8495 & 0.071 \\
C34 & $1.0486(8)$ & $0.0865(7)$ & $0.9064(5)$ & $0.064(2)$ \\
H34 & 1.1283 & 0.0612 & 0.8893 & 0.077
\end{tabular}




\begin{tabular}{|c|c|c|c|c|}
\hline C35 & $1.0170(8)$ & $0.1681(7)$ & $0.9549(5)$ & $0.067(2)$ \\
\hline H35 & 1.0755 & 0.1985 & 0.9708 & 0.081 \\
\hline C36 & $0.9022(8)$ & $0.2060(7)$ & $0.9807(5)$ & $0.061(2)$ \\
\hline H36 & 0.8820 & 0.2617 & 1.0145 & 0.073 \\
\hline C37 & $0.8153(7)$ & $0.1622(6)$ & $0.9570(5)$ & $0.055(2)$ \\
\hline C38 & $0.6866(7)$ & $0.1921(6)$ & $0.9812(5)$ & $0.055(2)$ \\
\hline H38 & 0.6654 & 0.2497 & 1.0180 & 0.066 \\
\hline C39 & $0.6197(7)$ & $0.2134(6)$ & $0.8968(5)$ & $0.051(2)$ \\
\hline C40 & $0.5384(7)$ & $0.2985(6)$ & $0.8693(5)$ & $0.058(2)$ \\
\hline $\mathrm{H} 40$ & 0.5175 & 0.3532 & 0.9043 & 0.069 \\
\hline C41 & $0.4839(7)$ & $0.3066(6)$ & $0.7884(5)$ & $0.054(2)$ \\
\hline N42 & $0.4026(6)$ & $0.3940(5)$ & $0.7631(4)$ & $0.0581(19)$ \\
\hline C43 & $0.3551(7)$ & $0.3973(6)$ & $0.6863(5)$ & $0.054(2)$ \\
\hline C44 & $0.2671(7)$ & $0.4907(6)$ & $0.6558(5)$ & $0.057(2)$ \\
\hline C45 & $0.2353(8)$ & $0.5714(6)$ & $0.7053(5)$ & $0.065(2)$ \\
\hline H45 & 0.2717 & 0.5661 & 0.7593 & 0.078 \\
\hline C46 & $0.1520(8)$ & $0.6602(6)$ & $0.6790(5)$ & $0.067(2)$ \\
\hline C47 & $0.1002(8)$ & $0.6641(6)$ & $0.5990(5)$ & $0.064(2)$ \\
\hline $\mathrm{H} 47$ & 0.0413 & 0.7228 & 0.5796 & 0.077 \\
\hline C48 & $0.1319(7)$ & $0.5864(6)$ & $0.5481(5)$ & $0.056(2)$ \\
\hline C49 & $0.0772(7)$ & $0.5915(6)$ & $0.4639(5)$ & $0.059(2)$ \\
\hline N50 & $-0.0021(6)$ & $0.6784(5)$ & $0.4381(4)$ & $0.067(2)$ \\
\hline C51 & $-0.0505(7)$ & $0.6839(7)$ & $0.3615(5)$ & $0.064(2)$ \\
\hline C52 & $-0.1360(8)$ & $0.7765(7)$ & $0.3331(6)$ & $0.072(2)$ \\
\hline C53 & $-0.1855(9)$ & $0.7805(8)$ & $0.2552(6)$ & $0.076(3)$ \\
\hline H53 & -0.2430 & 0.8399 & 0.2356 & 0.091 \\
\hline C54 & $-0.1554(8)$ & $0.7014(7)$ & $0.2033(6)$ & $0.077(3)$ \\
\hline H54 & -0.1913 & 0.7092 & 0.1489 & 0.092 \\
\hline C55 & $0.2490(7)$ & $0.4122(6)$ & $0.5227(5)$ & $0.0503(19)$ \\
\hline C56 & $0.2168(7)$ & $0.4950(6)$ & $0.5750(5)$ & $0.052(2)$ \\
\hline C57 & $0.3750(14)$ & $0.1669(10)$ & $0.3586(9)$ & $0.079(5)$ \\
\hline C571 & $0.312(2)$ & $0.187(2)$ & $0.2725(9)$ & $0.089(8)$ \\
\hline H57A & 0.3449 & 0.1309 & 0.2374 & 0.133 \\
\hline H57B & 0.3225 & 0.2484 & 0.2437 & 0.133 \\
\hline H57C & 0.2273 & 0.1942 & 0.2813 & 0.133 \\
\hline C572 & $0.364(2)$ & $0.0672(12)$ & $0.4014(13)$ & $0.108(8)$ \\
\hline H57D & 0.4080 & 0.0124 & 0.3682 & 0.162 \\
\hline H57E & 0.2809 & 0.0679 & 0.4041 & 0.162 \\
\hline H57F & 0.3968 & 0.0579 & 0.4590 & 0.162 \\
\hline C573 & $0.5062(13)$ & $0.1581(13)$ & $0.3429(10)$ & $0.092(6)$ \\
\hline H57G & 0.5375 & 0.1076 & 0.3023 & 0.138 \\
\hline $\mathrm{H} 57 \mathrm{H}$ & 0.5497 & 0.1385 & 0.3966 & 0.138 \\
\hline H57I & 0.5152 & 0.2226 & 0.3197 & 0.138 \\
\hline C58 & $0.1181(9)$ & $0.7474(7)$ & $0.7348(6)$ & $0.084(3)$ \\
\hline C581 & $0.0254(10)$ & $0.7294(10)$ & $0.7982(7)$ & $0.109(4)$ \\
\hline H58A & -0.0015 & 0.7869 & 0.8326 & 0.163 \\
\hline H58B & 0.0599 & 0.6693 & 0.8354 & 0.163 \\
\hline H58C & -0.0418 & 0.7204 & 0.7673 & 0.163 \\
\hline C582 & $0.0666(18)$ & $0.8459(8)$ & $0.6832(8)$ & $0.196(9)$ \\
\hline H58D & -0.0013 & 0.8862 & 0.7133 & 0.294 \\
\hline H58E & 0.0412 & 0.8328 & 0.6275 & 0.294 \\
\hline H58F & 0.1268 & 0.8822 & 0.6755 & 0.294 \\
\hline C583 & $0.2213(11)$ & $0.7565(11)$ & $0.7853(9)$ & $0.134(5)$ \\
\hline H58G & 0.1965 & 0.8155 & 0.8182 & 0.201 \\
\hline $\mathrm{H} 58 \mathrm{H}$ & 0.2850 & 0.7632 & 0.7464 & 0.201 \\
\hline H58I & 0.2496 & 0.6967 & 0.8241 & 0.201 \\
\hline O59 & $-0.0416(7)$ & $0.5314(6)$ & $0.1832(4)$ & $0.096(2)$ \\
\hline C59 & $-0.0991(14)$ & $0.5393(10)$ & $0.1030(7)$ & $0.126(5)$ \\
\hline H59A & -0.0664 & 0.4779 & 0.0742 & 0.189 \\
\hline H59B & -0.0864 & 0.5963 & 0.0678 & 0.189 \\
\hline H59C & -0.1839 & 0.5491 & 0.1120 & 0.189 \\
\hline
\end{tabular}




\begin{tabular}{|c|c|c|c|c|}
\hline $\mathrm{O} 60$ & $-0.1584(6)$ & $0.8485(5)$ & $0.3866(4)$ & $0.089(2)$ \\
\hline C60 & $-0.2446(11)$ & $0.9392(8)$ & $0.3612(7)$ & $0.103(4)$ \\
\hline $\mathrm{H} 60 \mathrm{~A}$ & -0.2524 & 0.9863 & 0.4054 & 0.154 \\
\hline H60B & -0.3207 & 0.9256 & 0.3530 & 0.154 \\
\hline $\mathrm{H} 60 \mathrm{C}$ & -0.2201 & 0.9681 & 0.3078 & 0.154 \\
\hline C57B & $0.3333(18)$ & $0.1571(13)$ & $0.3692(13)$ & $0.081(8)$ \\
\hline C574 & $0.437(3)$ & $0.075(2)$ & $0.408(2)$ & $0.120(14)$ \\
\hline H57J & 0.4530 & 0.0170 & 0.3743 & 0.180 \\
\hline H57K & 0.4188 & 0.0568 & 0.4665 & 0.180 \\
\hline H57L & 0.5075 & 0.1003 & 0.4074 & 0.180 \\
\hline C575 & $0.358(4)$ & $0.180(3)$ & $0.2755(14)$ & $0.120(16)$ \\
\hline H57M & 0.3730 & 0.1181 & 0.2458 & 0.179 \\
\hline $\mathrm{H} 57 \mathrm{~N}$ & 0.4271 & 0.2055 & 0.2706 & 0.179 \\
\hline H57O & 0.2893 & 0.2294 & 0.2500 & 0.179 \\
\hline C576 & $0.223(2)$ & $0.1196(19)$ & $0.3757(19)$ & $0.097(10)$ \\
\hline H57P & 0.2302 & 0.0675 & 0.3361 & 0.145 \\
\hline H57Q & 0.1535 & 0.1751 & 0.3612 & 0.145 \\
\hline H57R & 0.2158 & 0.0921 & 0.4338 & 0.145 \\
\hline C61 & $0.328(2)$ & $0.4416(10)$ & $0.0782(15)$ & $0.155(11)$ \\
\hline H61 & 0.3836 & 0.4478 & 0.1230 & 0.186 \\
\hline Cl1 & $0.3430(5)$ & $0.5246(3)$ & $-0.0053(4)$ & $0.106(2)$ \\
\hline $\mathrm{Cl} 2$ & $0.1899(5)$ & $0.4709(5)$ & $0.1253(4)$ & $0.114(2)$ \\
\hline $\mathrm{Cl} 3$ & $0.3728(8)$ & $0.3199(4)$ & $0.0543(4)$ & $0.137(3)$ \\
\hline C61B & $0.320(3)$ & $0.4517(14)$ & $0.107(3)$ & $0.178(16)$ \\
\hline H61B & 0.3739 & 0.4553 & 0.0579 & 0.213 \\
\hline Cl1B & $0.411(2)$ & $0.4256(17)$ & $0.192(2)$ & $0.39(2)$ \\
\hline $\mathrm{Cl} 2 \mathrm{~B}$ & $0.2232(14)$ & $0.5697(11)$ & $0.1026(13)$ & $0.256(10)$ \\
\hline Cl3B & $0.260(2)$ & $0.3580(13)$ & $0.0837(10)$ & $0.244(10)$ \\
\hline C62B & $0.1221(17)$ & $0.8680(16)$ & $0.4276(11)$ & $0.105(12)$ \\
\hline H62B & 0.0814 & 0.8161 & 0.4468 & 0.126 \\
\hline Cl4B & $0.1085(10)$ & $0.8846(9)$ & $0.3177(6)$ & $0.089(3)$ \\
\hline Cl5B & $0.2669(13)$ & $0.8224(15)$ & $0.4599(9)$ & $0.147(7)$ \\
\hline Cl6B & $0.0500(12)$ & $0.9755(7)$ & $0.4756(5)$ & $0.151(5)$ \\
\hline C70 & $0.120(3)$ & $0.878(3)$ & $0.3585(17)$ & $0.125(12)$ \\
\hline $\mathrm{H} 70 \mathrm{~A}$ & 0.0520 & 0.8853 & 0.3211 & 0.188 \\
\hline $\mathrm{H} 70 \mathrm{~B}$ & 0.1622 & 0.9266 & 0.3397 & 0.188 \\
\hline $\mathrm{H} 70 \mathrm{C}$ & 0.0923 & 0.8880 & 0.4169 & 0.188 \\
\hline C71 & $0.2049(11)$ & $0.7696(8)$ & $0.3548(8)$ & $0.103(5)$ \\
\hline C72 & $0.2841(12)$ & $0.7256(11)$ & $0.4200(7)$ & $0.108(6)$ \\
\hline C73 & $0.3575(10)$ & $0.6295(11)$ & $0.4145(8)$ & $0.104(5)$ \\
\hline H73 & 0.4116 & 0.5994 & 0.4591 & 0.124 \\
\hline C74 & $0.3517(10)$ & $0.5774(8)$ & $0.3438(9)$ & $0.089(4)$ \\
\hline C75 & $0.2725(11)$ & $0.6214(9)$ & $0.2786(7)$ & $0.101(5)$ \\
\hline $\mathrm{H} 75$ & 0.2685 & 0.5858 & 0.2303 & 0.121 \\
\hline C76 & $0.1991(10)$ & $0.7175(9)$ & $0.2841(7)$ & $0.090(5)$ \\
\hline $\mathrm{H} 76$ & 0.1450 & 0.7475 & 0.2395 & 0.108 \\
\hline N77 & $0.291(3)$ & $0.7842(15)$ & $0.4862(16)$ & $0.112(7)$ \\
\hline $\mathrm{O} 11$ & $0.234(4)$ & $0.8718(19)$ & $0.487(2)$ & $0.26(2)$ \\
\hline $\mathrm{O} 12$ & $0.348(2)$ & $0.7383(18)$ & $0.5486(12)$ & $0.184(8)$ \\
\hline N78 & $0.4289(15)$ & $0.4814(13)$ & $0.3353(11)$ & $0.101(5)$ \\
\hline 013 & $0.4899(12)$ & $0.4430(12)$ & $0.3967(10)$ & $0.134(6)$ \\
\hline O14 & $0.4385(18)$ & $0.4370(13)$ & $0.2701(13)$ & $0.143(6)$ \\
\hline
\end{tabular}




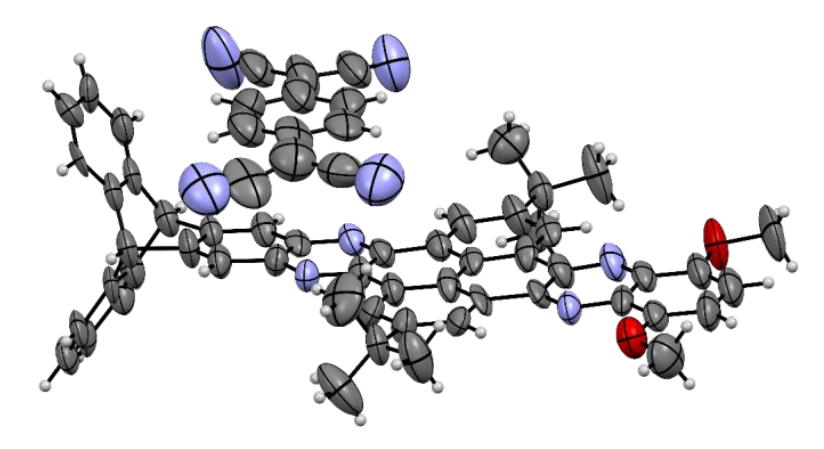

Figure S13. Thermal atomic displacement ellipsoid plot of the asymmetric unit of QPP-OMe/TCNQ. The ellipsoids of non-hydrogen atoms are drawn at the $50 \%$ probability level and hydrogen atoms are represented by a sphere of arbitrary size.

Table S3. Crystal data and structure refinement for QPP-OMe/TCNQ.

\begin{tabular}{|c|c|c|}
\hline $\begin{array}{l}\text { CCDC } \\
\text { Solvent } \\
\text { Empirical formula } \\
\text { Formula weight } \\
\text { Temperature } \\
\text { Wavelength } \\
\text { Crystal system }\end{array}$ & $\begin{array}{l}2040321 \\
\mathrm{CHCl}_{3} / \mathrm{MeOH} \\
\mathrm{C}_{64} \mathrm{H}_{46} \mathrm{~N}_{8} \mathrm{O}_{2} \\
959.09 \\
100(2) \mathrm{K} \\
1.54178 \AA \\
\text { triclinic }\end{array}$ & \\
\hline $\begin{array}{l}\text { Space group } \\
Z\end{array}$ & \multicolumn{2}{|l|}{$P \overline{1}$} \\
\hline Unit cell dimensions & $\begin{array}{l}a=11.694(2) \AA \\
b=14.705(3) \AA \\
c=15.582(3) \AA\end{array}$ & $\begin{array}{l}\alpha=84.859(16) \text { deg. } \\
\beta=84.363(16) \text { deg. } \\
\gamma=69.541(15) \text { deg. }\end{array}$ \\
\hline Volume & $2493.9(9) \AA^{3}$ & \\
\hline Density (calculated) & $1.28 \mathrm{~g} / \mathrm{cm}^{3}$ & \\
\hline Absorption coefficient & $0.62 \mathrm{~mm}^{-1}$ & \\
\hline Crystal shape & needle & \\
\hline Crystal size & $0.232 \times 0.034 \times 0$ & $0 \mathrm{~mm}^{3}$ \\
\hline Crystal colour & black & \\
\hline Theta range for data collection & 4.0 to 40.4 deg. & \\
\hline Index ranges & $-8 \leq \mathrm{h} \leq 9,-12 \leq \mathrm{k} \leq 1$ & $3 \leq 1 \leq 13$ \\
\hline Reflections collected & 14341 & \\
\hline Independent reflections & $3070(\mathrm{R}(\mathrm{int})=0$. & \\
\hline Observed reflections & $1394(\mathrm{I}>2 \sigma(\mathrm{I}))$ & \\
\hline Absorption correction & Semi-empirical fr & equivalents \\
\hline Max. and min. transmission & 2.55 and 0.32 & \\
\hline Refinement method & Full-matrix least- & ares on $\mathrm{F}^{2}$ \\
\hline Data/restraints/parameters & $3070 / 815 / 667$ & \\
\hline Goodness-of-fit on $\mathrm{F}^{2}$ & 1.49 & \\
\hline Final $R$ indices $(I>2$ sigma $(I))$ & $\mathrm{R} 1=0.186, \mathrm{wR} 2$ & .404 \\
\hline Largest diff. peak and hole & 0.90 and $-0.56 \mathrm{e}$ & \\
\hline
\end{tabular}

Table S4. Atomic coordinates and equivalent isotropic displacement parameters $\left(\AA^{2}\right)$ for QPPOMe/TCNQ. $U_{\text {eq }}$ is defined as one third of the trace of the orthogonalized $U_{i j}$ tensor.

\begin{tabular}{lllll}
\hline & & & & \\
Atom & $x$ & $y$ & $z$ & $U_{\text {eq }}$ \\
\hline & & & & \\
C11 & $-0.0633(19)$ & $0.6184(16)$ & $0.2361(14)$ & $0.069(7)$ \\
C12 & $-0.0129(16)$ & $0.6092(13)$ & $0.3150(12)$ & $0.061(6)$ \\
N13 & $0.0646(14)$ & $0.5211(11)$ & $0.3423(10)$ & $0.068(5)$
\end{tabular}




\begin{tabular}{|c|c|c|c|c|}
\hline C14 & $0.1092(17)$ & $0.5146(12)$ & $0.4185(12)$ & $0.062(6)$ \\
\hline C15 & $0.1964(17)$ & $0.4208(13)$ & $0.4489(12)$ & $0.060(6)$ \\
\hline C16 & $0.2300(16)$ & $0.3384(13)$ & $0.3987(13)$ & $0.066(6)$ \\
\hline H16 & 0.1951 & 0.3431 & 0.3453 & 0.079 \\
\hline C17 & $0.3132(17)$ & $0.2516(13)$ & $0.4276(12)$ & $0.074(7)$ \\
\hline C18 & $0.3599(17)$ & $0.2430(14)$ & $0.5077(12)$ & $0.068(7)$ \\
\hline H18 & 0.4128 & 0.1816 & 0.5288 & 0.082 \\
\hline C19 & $0.3297(18)$ & $0.3241(13)$ & $0.5578(12)$ & $0.065(6)$ \\
\hline $\mathrm{C} 20$ & $0.3796(17)$ & $0.3181(13)$ & $0.6402(11)$ & $0.064(6)$ \\
\hline N21 & $0.4653(14)$ & $0.2331(11)$ & $0.6639(10)$ & $0.066(5)$ \\
\hline C22 & $0.5116(17)$ & $0.2285(13)$ & $0.7415(12)$ & $0.061(6)$ \\
\hline C23 & $0.6055(17)$ & $0.1391(13)$ & $0.7673(12)$ & $0.066(6)$ \\
\hline $\mathrm{H} 23$ & 0.6360 & 0.0866 & 0.7303 & 0.079 \\
\hline C24 & $0.6479(18)$ & $0.1325(12)$ & $0.8448(12)$ & $0.072(7)$ \\
\hline C25 & $0.7461(16)$ & $0.0417(13)$ & $0.8858(12)$ & $0.068(5)$ \\
\hline $\mathrm{H} 25$ & 0.7779 & -0.0134 & 0.8467 & 0.082 \\
\hline C26 & $0.6884(17)$ & $0.0148(13)$ & $0.9707(13)$ & $0.062(6)$ \\
\hline C27 & $0.6737(19)$ & $-0.0740(14)$ & $0.9955(14)$ & $0.075(7)$ \\
\hline $\mathrm{H} 27$ & 0.6977 & -0.1252 & 0.9572 & 0.091 \\
\hline C28 & $0.624(2)$ & $-0.0844(16)$ & $1.0767(14)$ & $0.079(7)$ \\
\hline $\mathrm{H} 28$ & 0.6139 & -0.1446 & 1.0961 & 0.095 \\
\hline C29 & $0.5866(17)$ & $-0.0089(15)$ & $1.1313(14)$ & $0.074(7)$ \\
\hline $\mathrm{H} 29$ & 0.5532 & -0.0191 & 1.1879 & 0.089 \\
\hline C30 & $0.5963(19)$ & $0.0816(15)$ & $1.1066(13)$ & $0.081(7)$ \\
\hline $\mathrm{H} 30$ & 0.5688 & 0.1339 & 1.1439 & 0.097 \\
\hline C31 & $0.6490(19)$ & $0.0901(14)$ & $1.0241(13)$ & $0.072(6)$ \\
\hline C32 & $0.8450(19)$ & $0.0807(14)$ & $0.9058(12)$ & $0.064(6)$ \\
\hline C33 & $0.9684(18)$ & $0.0403(13)$ & $0.8785(12)$ & $0.060(6)$ \\
\hline H33 & 0.9980 & -0.0141 & 0.8436 & 0.072 \\
\hline C34 & $1.044(2)$ & $0.0832(14)$ & $0.9048(13)$ & $0.070(7)$ \\
\hline H34 & 1.1275 & 0.0601 & 0.8835 & 0.084 \\
\hline C35 & $1.0079(19)$ & $0.1579(14)$ & $0.9604(13)$ & $0.071(7)$ \\
\hline H35 & 1.0654 & 0.1828 & 0.9793 & 0.085 \\
\hline C36 & $0.8859(19)$ & $0.1951(15)$ & $0.9872(14)$ & $0.081(7)$ \\
\hline H36 & 0.8565 & 0.2488 & 1.0229 & 0.097 \\
\hline C37 & $0.8073(18)$ & $0.1538(14)$ & $0.9620(12)$ & $0.063(6)$ \\
\hline C38 & $0.6718(17)$ & $0.1835(13)$ & $0.9869(11)$ & $0.070(5)$ \\
\hline H38 & 0.6425 & 0.2369 & 1.0279 & 0.084 \\
\hline C39 & $0.6055(16)$ & $0.2110(12)$ & $0.9009(11)$ & $0.060(6)$ \\
\hline C40 & $0.5201(17)$ & $0.2951(13)$ & $0.8774(11)$ & $0.062(6)$ \\
\hline $\mathrm{H} 40$ & 0.4915 & 0.3470 & 0.9151 & 0.075 \\
\hline C41 & $0.4722(16)$ & $0.3054(13)$ & $0.7940(11)$ & $0.054(6)$ \\
\hline N42 & $0.3867(14)$ & $0.3921(11)$ & $0.7701(10)$ & $0.067(5)$ \\
\hline C43 & $0.3438(17)$ & $0.3989(12)$ & $0.6923(12)$ & $0.063(6)$ \\
\hline C44 & $0.2606(17)$ & $0.4924(13)$ & $0.6626(12)$ & $0.063(6)$ \\
\hline C45 & $0.2280(19)$ & $0.5756(13)$ & $0.7109(13)$ & $0.082(7)$ \\
\hline $\mathrm{H} 45$ & 0.2592 & 0.5706 & 0.7658 & 0.099 \\
\hline C46 & $0.1507(19)$ & $0.6647(13)$ & $0.6787(12)$ & $0.083(7)$ \\
\hline C47 & $0.1011(17)$ & $0.6716(14)$ & $0.6009(12)$ & $0.062(6)$ \\
\hline $\mathrm{H} 47$ & 0.0454 & 0.7321 & 0.5803 & 0.075 \\
\hline C48 & $0.1325(17)$ & $0.5892(14)$ & $0.5512(12)$ & $0.066(6)$ \\
\hline C49 & $0.0783(19)$ & $0.5974(13)$ & $0.4691(11)$ & $0.073(7)$ \\
\hline N50 & $0.0049(16)$ & $0.6850(12)$ & $0.4407(11)$ & $0.083(6)$ \\
\hline C51 & $-0.0403(18)$ & $0.6916(13)$ & $0.3631(12)$ & $0.065(6)$ \\
\hline C52 & $-0.123(2)$ & $0.7800(16)$ & $0.3327(15)$ & $0.082(7)$ \\
\hline C53 & $-0.173(2)$ & $0.7864(15)$ & $0.2563(15)$ & $0.092(8)$ \\
\hline H53 & -0.2277 & 0.8471 & 0.2355 & 0.111 \\
\hline C54 & $-0.143(2)$ & $0.7036(16)$ & $0.2072(14)$ & $0.090(8)$ \\
\hline H54 & -0.1797 & 0.7083 & 0.1544 & 0.108 \\
\hline C55 & $0.2435(17)$ & $0.4139(12)$ & $0.5291(11)$ & $0.064(6)$ \\
\hline C56 & $0.2116(16)$ & $0.4993(12)$ & $0.5814(11)$ & $0.060(6)$ \\
\hline
\end{tabular}




\begin{tabular}{|c|c|c|c|c|}
\hline C57 & $0.3607(17)$ & $0.1624(13)$ & $0.3710(11)$ & $0.078(6)$ \\
\hline C571 & $0.308(2)$ & $0.1811(16)$ & $0.2859(12)$ & $0.121(10)$ \\
\hline H57A & 0.3213 & 0.2385 & 0.2560 & 0.181 \\
\hline H57B & 0.3478 & 0.1246 & 0.2512 & 0.181 \\
\hline H57C & 0.2200 & 0.1925 & 0.2942 & 0.181 \\
\hline C572 & $0.346(3)$ & $0.0761(14)$ & $0.4225(15)$ & $0.144(10)$ \\
\hline H57D & 0.3826 & 0.0683 & 0.4777 & 0.216 \\
\hline H57E & 0.2584 & 0.0856 & 0.4333 & 0.216 \\
\hline H57F & 0.3862 & 0.0176 & 0.3903 & 0.216 \\
\hline C573 & $0.4977(18)$ & $0.1381(19)$ & $0.3502(16)$ & $0.150(11)$ \\
\hline H57G & 0.5391 & 0.1247 & 0.4041 & 0.225 \\
\hline $\mathrm{H} 57 \mathrm{H}$ & 0.5295 & 0.0806 & 0.3159 & 0.225 \\
\hline H57I & 0.5128 & 0.1932 & 0.3173 & 0.225 \\
\hline C58 & $0.1163(16)$ & $0.7543(12)$ & $0.7342(11)$ & $0.077(6)$ \\
\hline C581 & $0.056(2)$ & $0.8472(12)$ & $0.6819(13)$ & $0.125(10)$ \\
\hline H58A & 0.1118 & 0.8545 & 0.6328 & 0.188 \\
\hline H58B & -0.0192 & 0.8451 & 0.6605 & 0.188 \\
\hline H58C & 0.0355 & 0.9025 & 0.7183 & 0.188 \\
\hline C582 & $0.0297(16)$ & $0.7441(14)$ & $0.8089(12)$ & $0.089(7)$ \\
\hline H58D & -0.0443 & 0.7403 & 0.7872 & 0.133 \\
\hline H58E & 0.0691 & 0.6847 & 0.8442 & 0.133 \\
\hline H58F & 0.0076 & 0.8005 & 0.8440 & 0.133 \\
\hline C583 & $0.2286(18)$ & $0.7599(16)$ & $0.7692(14)$ & $0.110(8)$ \\
\hline H58G & 0.2864 & 0.7666 & 0.7211 & 0.165 \\
\hline $\mathrm{H} 58 \mathrm{H}$ & 0.2057 & 0.8163 & 0.8045 & 0.165 \\
\hline H58I & 0.2672 & 0.7005 & 0.8047 & 0.165 \\
\hline O59 & $-0.0295(14)$ & $0.5354(11)$ & $0.1907(10)$ & $0.093(5)$ \\
\hline C59 & $-0.085(2)$ & $0.5421(18)$ & $0.1105(16)$ & $0.116(10)$ \\
\hline H59A & -0.0536 & 0.4787 & 0.0850 & 0.174 \\
\hline H59B & -0.1741 & 0.5614 & 0.1218 & 0.174 \\
\hline H59C & -0.0654 & 0.5907 & 0.0705 & 0.174 \\
\hline O60 & $-0.1375(16)$ & $0.8566(11)$ & $0.3819(11)$ & $0.116(6)$ \\
\hline C60 & $-0.214(3)$ & $0.9525(16)$ & $0.3509(19)$ & $0.145(12)$ \\
\hline $\mathrm{H} 60 \mathrm{~A}$ & -0.2169 & 1.0004 & 0.3916 & 0.218 \\
\hline $\mathrm{H} 60 \mathrm{~B}$ & -0.1806 & 0.9692 & 0.2941 & 0.218 \\
\hline $\mathrm{H} 60 \mathrm{C}$ & -0.2973 & 0.9526 & 0.3462 & 0.218 \\
\hline C71 & $0.704(2)$ & $0.3304(17)$ & $0.6285(17)$ & $0.112(9)$ \\
\hline C72 & $0.625(2)$ & $0.4296(16)$ & $0.6284(15)$ & $0.114(9)$ \\
\hline $\mathrm{H} 72$ & 0.5771 & 0.4575 & 0.5808 & 0.137 \\
\hline C73 & $0.619(2)$ & $0.4844(17)$ & $0.6956(15)$ & $0.116(9)$ \\
\hline $\mathrm{H} 73$ & 0.5659 & 0.5503 & 0.6965 & 0.140 \\
\hline C74 & $0.694(2)$ & $0.4386(16)$ & $0.7648(18)$ & $0.114(9)$ \\
\hline C75 & $0.773(2)$ & $0.3401(15)$ & $0.7666(16)$ & $0.110(9)$ \\
\hline $\mathrm{H} 75$ & 0.8224 & 0.3122 & 0.8134 & 0.132 \\
\hline C76 & $0.775(2)$ & $0.2867(16)$ & $0.6998(15)$ & $0.116(9)$ \\
\hline $\mathrm{H} 76$ & 0.8232 & 0.2196 & 0.7006 & 0.139 \\
\hline C77 & $0.703(3)$ & $0.2755(19)$ & $0.558(2)$ & $0.142(12)$ \\
\hline C78 & $0.681(2)$ & $0.4939(17)$ & $0.8373(19)$ & $0.127(10)$ \\
\hline $\mathrm{C} 80$ & $0.756(3)$ & $0.172(2)$ & $0.5566(18)$ & $0.143(13)$ \\
\hline N80 & $0.827(3)$ & $0.093(2)$ & $0.559(2)$ & $0.176(12)$ \\
\hline C81 & $0.629(3)$ & $0.318(2)$ & $0.4867(18)$ & $0.121(10)$ \\
\hline N81 & $0.566(3)$ & $0.352(2)$ & $0.4306(18)$ & $0.176(12)$ \\
\hline C82 & $0.596(3)$ & $0.5913(19)$ & $0.8372(19)$ & $0.124(10)$ \\
\hline N82 & $0.529(3)$ & $0.6684(17)$ & $0.8509(15)$ & $0.151(11)$ \\
\hline C83 & $0.749(3)$ & $0.4544(19)$ & $0.9111(18)$ & $0.118(10)$ \\
\hline N83 & $0.800(3)$ & $0.4327(18)$ & $0.974(2)$ & $0.196(15)$ \\
\hline
\end{tabular}




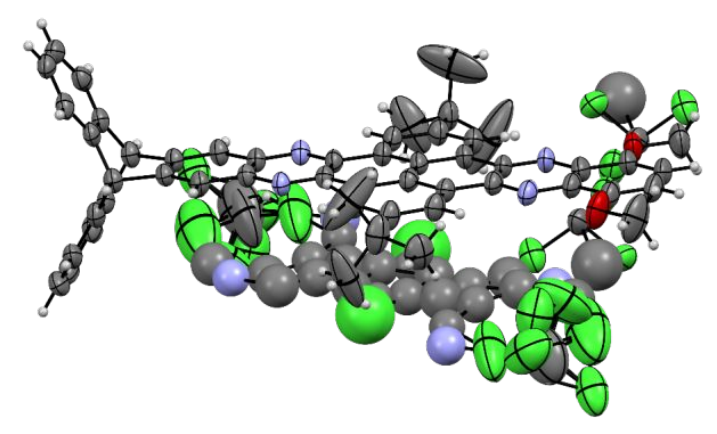

Figure S14. Thermal atomic displacement ellipsoid plot of the asymmetric unit of QPP-OMe/FTCNQ. The ellipsoids of non-hydrogen atoms are drawn at the $50 \%$ probability level and hydrogen atoms are represented by a sphere of arbitrary size. Disordered atoms have not been squeezed and are represented by dummy atoms shown as ball model.

Table S5. Crystal data and structure refinement for QPP-OMe/TCNQ.

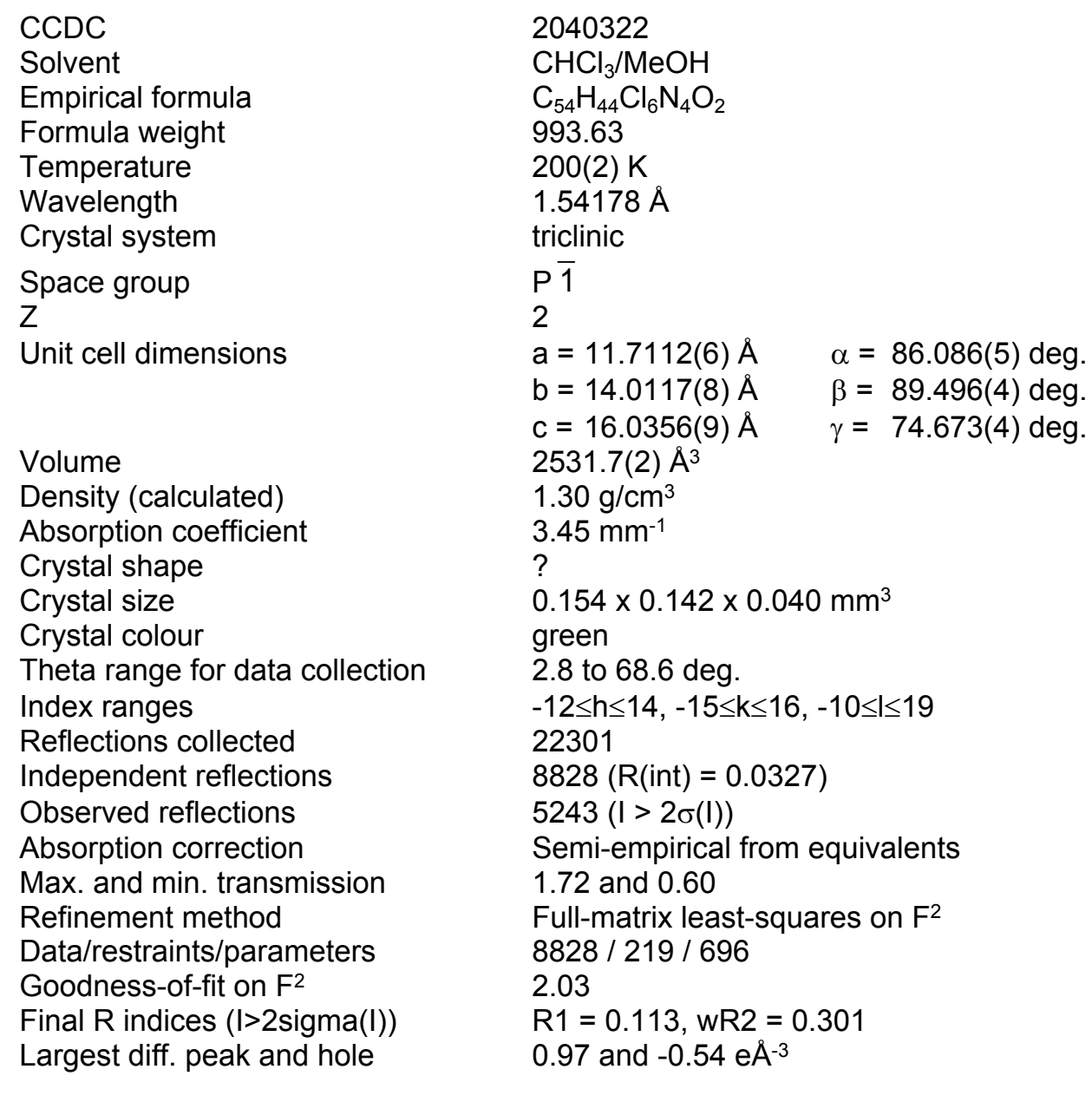

Table S6. Atomic coordinates and equivalent isotropic displacement parameters $\left(\AA^{2}\right)$ for QPPOMe/FTCNQ. $U_{\text {eq }}$ is defined as one third of the trace of the orthogonalized $U_{i j}$ tensor.

\begin{tabular}{ccccc} 
Atom & $\mathrm{x}$ & $\mathrm{y}$ & $\mathrm{z}$ & $\mathrm{U}_{\text {eq }}$ \\
\hline $\mathrm{C} 11$ & $1.1204(4)$ & $0.2378(4)$ & $0.6683(3)$ & $0.0637(14)$ \\
$\mathrm{C} 12$ & $1.0367(4)$ & $0.3285(4)$ & $0.6392(3)$ & $0.0556(12)$
\end{tabular}




\begin{tabular}{|c|c|c|c|c|}
\hline N13 & $0.9891(3)$ & $0.3335(3)$ & $0.5614(2)$ & $0.0546(10)$ \\
\hline C14 & $0.9128(3)$ & $0.4174(3)$ & $0.5357(2)$ & $0.0470(10)$ \\
\hline C15 & $0.8608(3)$ & $0.4248(3)$ & $0.4511(3)$ & $0.0474(11)$ \\
\hline C16 & $0.8912(4)$ & $0.3458(3)$ & $0.4010(3)$ & $0.0537(11)$ \\
\hline $\mathrm{H} 16$ & 0.9473 & 0.2869 & 0.4216 & 0.064 \\
\hline C17 & $0.8429(4)$ & $0.3487(3)$ & $0.3214(3)$ & $0.0531(11)$ \\
\hline C18 & $0.7620(4)$ & $0.4380(3)$ & $0.2937(3)$ & $0.0509(11)$ \\
\hline H18 & 0.7282 & 0.4435 & 0.2394 & 0.061 \\
\hline C19 & $0.7296(3)$ & $0.5181(3)$ & $0.3421(2)$ & $0.0441(10)$ \\
\hline C20 & $0.6433(3)$ & $0.6107(3)$ & $0.3114(2)$ & $0.0417(10)$ \\
\hline N21 & $0.5967(3)$ & $0.6127(2)$ & $0.2355(2)$ & $0.0449(8)$ \\
\hline C22 & $0.5161(3)$ & $0.6976(3)$ & $0.2086(2)$ & $0.0421(10)$ \\
\hline C23 & $0.4616(3)$ & $0.7040(3)$ & $0.1291(3)$ & $0.0454(10)$ \\
\hline $\mathrm{H} 23$ & 0.4824 & 0.6494 & 0.0948 & 0.054 \\
\hline C24 & $0.3796(3)$ & $0.7886(3)$ & $0.1019(2)$ & $0.0414(9)$ \\
\hline C25 & $0.3115(3)$ & $0.8088(3)$ & $0.0195(2)$ & $0.0458(10)$ \\
\hline $\mathrm{H} 25$ & 0.3317 & 0.7507 & -0.0159 & 0.055 \\
\hline C26 & $0.3388(3)$ & $0.9000(3)$ & $-0.0228(3)$ & $0.0435(10)$ \\
\hline C27 & $0.3855(4)$ & $0.9090(4)$ & $-0.1018(3)$ & $0.0525(11)$ \\
\hline $\mathrm{H} 27$ & 0.4045 & 0.8543 & -0.1360 & 0.063 \\
\hline C28 & $0.4039(4)$ & 0.9994(4) & $-0.1301(3)$ & $0.0576(12)$ \\
\hline $\mathrm{H} 28$ & 0.4344 & 1.0068 & -0.1846 & 0.069 \\
\hline C29 & $0.3789(4)$ & $1.0786(4)$ & $-0.0807(3)$ & $0.0563(12)$ \\
\hline $\mathrm{H} 29$ & 0.3931 & 1.1395 & -0.1014 & 0.068 \\
\hline C30 & $0.3333(3)$ & $1.0709(3)$ & $-0.0018(3)$ & $0.0506(11)$ \\
\hline $\mathrm{H} 30$ & 0.3170 & 1.1258 & 0.0322 & 0.061 \\
\hline C31 & $0.3115(3)$ & $0.9810(3)$ & $0.0275(2)$ & $0.0441(10)$ \\
\hline C32 & $0.1817(3)$ & $0.8386(3)$ & $0.0427(2)$ & $0.0444(10)$ \\
\hline C33 & $0.0944(4)$ & $0.7950(3)$ & $0.0199(3)$ & $0.0551(12)$ \\
\hline H33 & 0.1134 & 0.7389 & -0.0127 & 0.066 \\
\hline C34 & $-0.0199(4)$ & $0.8336(4)$ & $0.0449(3)$ & $0.0648(14)$ \\
\hline H34 & -0.0798 & 0.8038 & 0.0292 & 0.078 \\
\hline C35 & $-0.0495(4)$ & $0.9150(4)$ & $0.0925(3)$ & $0.0624(13)$ \\
\hline H35 & -0.1291 & 0.9407 & 0.1092 & 0.075 \\
\hline C36 & $0.0371(4)$ & $0.9592(4)$ & $0.1160(3)$ & $0.0542(12)$ \\
\hline H36 & 0.0174 & 1.0152 & 0.1488 & 0.065 \\
\hline C37 & $0.1527(4)$ & $0.9204(3)$ & $0.0909(3)$ & $0.0471(10)$ \\
\hline C38 & $0.2588(3)$ & $0.9593(3)$ & $0.1106(2)$ & $0.0439(10)$ \\
\hline H38 & 0.2380 & 1.0178 & 0.1455 & 0.053 \\
\hline C39 & $0.3497(3)$ & $0.8710(3)$ & $0.1522(2)$ & $0.0413(9)$ \\
\hline C40 & $0.3997(3)$ & $0.8675(3)$ & $0.2294(2)$ & $0.0431(10)$ \\
\hline $\mathrm{H} 40$ & 0.3782 & 0.9231 & 0.2625 & 0.052 \\
\hline C41 & $0.4844(3)$ & $0.7792(3)$ & $0.2595(2)$ & $0.0407(9)$ \\
\hline N42 & $0.5326(3)$ & $0.7756(2)$ & $0.3365(2)$ & $0.0425(8)$ \\
\hline C43 & $0.6107(3)$ & $0.6914(3)$ & $0.3623(2)$ & $0.0401(9)$ \\
\hline C44 & $0.6620(3)$ & $0.6844(3)$ & $0.4465(2)$ & $0.0428(10)$ \\
\hline C45 & $0.6279(4)$ & $0.7619(3)$ & $0.4979(3)$ & $0.0528(11)$ \\
\hline $\mathrm{H} 45$ & 0.5717 & 0.8208 & 0.4774 & 0.063 \\
\hline C46 & $0.6730(4)$ & $0.7568(3)$ & $0.5792(3)$ & $0.0563(12)$ \\
\hline C47 & $0.7563(4)$ & $0.6701(3)$ & $0.6060(3)$ & $0.0537(11)$ \\
\hline $\mathrm{H} 47$ & 0.7896 & 0.6651 & 0.6605 & 0.064 \\
\hline C48 & $0.7930(4)$ & $0.5906(3)$ & $0.5568(2)$ & $0.0472(10)$ \\
\hline C49 & $0.8797(4)$ & $0.4984(3)$ & $0.5869(3)$ & $0.0493(11)$ \\
\hline N50 & $0.9241(3)$ & $0.4940(3)$ & $0.6640(2)$ & $0.0544(10)$ \\
\hline C51 & $1.0044(4)$ & $0.4084(4)$ & $0.6901(3)$ & $0.0567(12)$ \\
\hline C52 & $1.0566(5)$ & $0.4005(4)$ & $0.7718(3)$ & $0.0671(14)$ \\
\hline C53 & $1.1360(5)$ & $0.3140(5)$ & $0.7978(3)$ & $0.0749(16)$ \\
\hline H53 & 1.1705 & 0.3077 & 0.8520 & 0.090 \\
\hline C54 & $1.1682(4)$ & $0.2342(4)$ & $0.7470(3)$ & $0.0694(15)$ \\
\hline H54 & 1.2249 & 0.1756 & 0.7674 & 0.083 \\
\hline C55 & $0.7797(3)$ & $0.5131(3)$ & $0.4228(3)$ & $0.0452(10)$ \\
\hline
\end{tabular}




\begin{tabular}{|c|c|c|c|c|}
\hline C56 & $0.7449(3)$ & $0.5965(3)$ & $0.4753(2)$ & $0.0441(10)$ \\
\hline C57 & $0.8769(4)$ & $0.2616(3)$ & $0.2670(3)$ & $0.0630(13)$ \\
\hline C571 & $0.8955(17)$ & $0.1676(6)$ & $0.3157(5)$ & $0.277(11)$ \\
\hline H57A & 0.9355 & 0.1136 & 0.2815 & 0.415 \\
\hline H57B & 0.8188 & 0.1581 & 0.3338 & 0.415 \\
\hline H57C & 0.9445 & 0.1678 & 0.3649 & 0.415 \\
\hline C572 & $0.9842(9)$ & $0.2679(9)$ & $0.2197(9)$ & $0.219(7)$ \\
\hline H57D & 0.9672 & 0.2724 & 0.1596 & 0.328 \\
\hline H57E & 1.0487 & 0.2085 & 0.2341 & 0.328 \\
\hline H57F & 1.0077 & 0.3269 & 0.2341 & 0.328 \\
\hline C573 & $0.7883(8)$ & $0.2627(7)$ & $0.2029(7)$ & $0.170(5)$ \\
\hline H57G & 0.8130 & 0.2023 & 0.1727 & 0.254 \\
\hline $\mathrm{H} 57 \mathrm{H}$ & 0.7806 & 0.3209 & 0.1637 & 0.254 \\
\hline H57I & 0.7118 & 0.2658 & 0.2295 & 0.254 \\
\hline C58 & $0.6305(6)$ & $0.8448(4)$ & $0.6325(3)$ & $0.0818(18)$ \\
\hline C581 & $0.6713(6)$ & $0.8240(5)$ & $0.7228(3)$ & $0.0883(18)$ \\
\hline H58A & 0.6381 & 0.8829 & 0.7537 & 0.132 \\
\hline H58B & 0.7579 & 0.8076 & 0.7254 & 0.132 \\
\hline H58C & 0.6443 & 0.7681 & 0.7477 & 0.132 \\
\hline C582 & $0.6651(16)$ & $0.9315(6)$ & $0.5915(5)$ & $0.225(8)$ \\
\hline H58D & 0.6182 & 0.9925 & 0.6145 & 0.338 \\
\hline H58E & 0.6506 & 0.9347 & 0.5312 & 0.338 \\
\hline $\mathrm{H} 58 \mathrm{~F}$ & 0.7494 & 0.9243 & 0.6020 & 0.338 \\
\hline C583 & $0.4941(7)$ & $0.8713(8)$ & $0.6371(6)$ & $0.180(6)$ \\
\hline H58G & 0.4647 & 0.9373 & 0.6582 & 0.270 \\
\hline $\mathrm{H} 58 \mathrm{H}$ & 0.4702 & 0.8222 & 0.6749 & 0.270 \\
\hline H58I & 0.4610 & 0.8712 & 0.5813 & 0.270 \\
\hline O59 & $1.1441(3)$ & $0.1646(3)$ & $0.6146(2)$ & $0.0738(11)$ \\
\hline C59 & $1.2259(5)$ & $0.0739(5)$ & $0.6428(4)$ & $0.0918(19)$ \\
\hline H59A & 1.2328 & 0.0256 & 0.6004 & 0.138 \\
\hline H59B & 1.1977 & 0.0481 & 0.6951 & 0.138 \\
\hline H59C & 1.3035 & 0.0856 & 0.6523 & 0.138 \\
\hline $\mathrm{O} 60$ & $1.0206(4)$ & $0.4813(3)$ & $0.8169(2)$ & $0.0899(13)$ \\
\hline C60 & $1.0728(9)$ & $0.4740(6)$ & $0.8974(4)$ & $0.133(3)$ \\
\hline $\mathrm{H} 60 \mathrm{~A}$ & 1.0481 & 0.5385 & 0.9217 & 0.200 \\
\hline $\mathrm{H} 60 \mathrm{~B}$ & 1.1592 & 0.4541 & 0.8927 & 0.200 \\
\hline $\mathrm{H} 60 \mathrm{C}$ & 1.0471 & 0.4244 & 0.9334 & 0.200 \\
\hline C61 & $0.8933(7)$ & $0.1286(8)$ & $0.5703(5)$ & $0.070(3)$ \\
\hline Cl1 & $0.9700(3)$ & $0.0200(3)$ & $0.5206(2)$ & $0.1023(11)$ \\
\hline $\mathrm{Cl} 2$ & $0.8985(3)$ & $0.0963(3)$ & $0.6785(2)$ & $0.0816(8)$ \\
\hline $\mathrm{Cl} 3$ & $0.7466(2)$ & $0.1617(2)$ & $0.5349(2)$ & $0.0854(9)$ \\
\hline C62 & $0.3429(18)$ & $0.4437(9)$ & $0.0726(11)$ & $0.227(10)$ \\
\hline H62 & 0.4144 & 0.4390 & 0.1079 & 0.272 \\
\hline $\mathrm{Cl} 4$ & $0.3394(4)$ & $0.5235(2)$ & $-0.0123(3)$ & $0.139(2)$ \\
\hline $\mathrm{Cl} 5$ & $0.3444(8)$ & $0.3287(4)$ & $0.0391(5)$ & $0.197(3)$ \\
\hline $\mathrm{Cl6}$ & $0.2159(6)$ & $0.4870(8)$ & $0.1286(6)$ & $0.247(4)$ \\
\hline C62B & $0.3333(19)$ & $0.4451(11)$ & $0.0887(18)$ & $0.231(13)$ \\
\hline H62B & 0.3820 & 0.4450 & 0.0372 & 0.277 \\
\hline Cl4B & $0.4336(7)$ & $0.4213(6)$ & $0.1738(7)$ & $0.245(6)$ \\
\hline Cl5B & $0.2645(9)$ & $0.3497(5)$ & $0.0819(5)$ & $0.168(3)$ \\
\hline Cl6B & $0.2399(13)$ & $0.5615(8)$ & $0.0895(13)$ & $0.328(9)$ \\
\hline C70 & $0.6083(17)$ & $0.4745(14)$ & $0.6752(13)$ & $0.125(5)$ \\
\hline C71 & $0.3804(18)$ & $0.5827(16)$ & $0.3925(13)$ & $0.131(6)$ \\
\hline C72 & $0.5437(13)$ & $0.4207(12)$ & $0.4635(10)$ & $0.086(4)$ \\
\hline C74 & $0.558(3)$ & $0.496(2)$ & $0.6124(19)$ & $0.166(8)$ \\
\hline C75 & $0.505(2)$ & $0.5330(17)$ & $0.5654(14)$ & $0.126(6)$ \\
\hline C76 & $0.4250(18)$ & $0.5722(15)$ & $0.4700(15)$ & $0.141(6)$ \\
\hline C78 & $0.4166(12)$ & $0.6588(11)$ & $0.5822(9)$ & $0.313(5)$ \\
\hline C79 & $0.308(2)$ & $0.668(2)$ & $0.3775(15)$ & $0.169(8)$ \\
\hline C80 & $0.701(2)$ & $0.3855(18)$ & $0.6895(14)$ & $0.146(7)$ \\
\hline C81 & $0.7621(13)$ & $0.3230(11)$ & $0.7191(9)$ & $0.122(4)$ \\
\hline
\end{tabular}




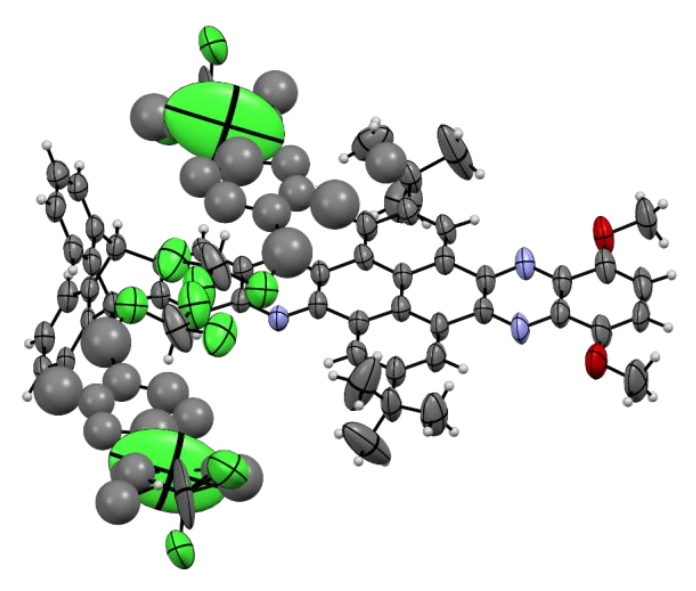

Figure S15. Thermal atomic displacement ellipsoid plot of the asymmetric unit of QPP-OMe/ $\mathbf{F}_{2} \mathbf{T C N Q}$. The ellipsoids of non-hydrogen atoms are drawn at the $50 \%$ probability level and hydrogen atoms are represented by a sphere of arbitrary size. Disordered atoms have not been squeezed and are represented by dummy atoms shown as ball model.

Table S7. Crystal data and structure refinement for QPP-OMe/ $\mathbf{F}_{2} \mathbf{T C N Q}$.

CCDC

Solvent

Empirical formula

Formula weight

Temperature

Wavelength

Crystal system

Space group

Z

Unit cell dimensions

Volume

Density (calculated)

Absorption coefficient

Crystal shape

Crystal size

Crystal colour

Theta range for data collection

Index ranges

Reflections collected

Independent reflections

Observed reflections

Absorption correction

Max. and min. transmission

Refinement method

Data/restraints/parameters

Goodness-of-fit on $\mathrm{F}^{2}$

Final $R$ indices ( $\mid>2$ sigma $(I))$
2040323

$\mathrm{CHCl}_{3} / \mathrm{MeOH}$

$\mathrm{C}_{52} \mathrm{H}_{42} \mathrm{Cl}_{0.50} \mathrm{~N}_{4} \mathrm{O}_{2}$

772.62

200(2) K

$1.54178 \AA$

triclinic

$\mathrm{P} \overline{1}$

2

$a=11.6277(16) \AA \quad \alpha=86.374(10) \mathrm{deg}$.

$\mathrm{b}=14.2580(19) \AA \quad \beta=88.404(10) \mathrm{deg}$.

$c=15.9300(19) \AA \quad \gamma=73.501(10) \mathrm{deg}$.

2527.1(6) $\AA^{3}$

$1.01 \mathrm{~g} / \mathrm{cm}^{3}$

$0.72 \mathrm{~mm}^{-1}$

plank

$0.089 \times 0.042 \times 0.015 \mathrm{~mm}^{3}$

green

4.0 to 50.4 deg.

$-11 \leq h \leq 11,-14 \leq k \leq 14,-13 \leq 1 \leq 15$

14505

$5236(\mathrm{R}$ (int) $=0.0846)$

$2453(I>2 \sigma(I))$

Semi-empirical from equivalents

1.58 and 0.54

Full-matrix least-squares on $\mathrm{F}^{2}$

5236 / 603 / 653

1.31

$\mathrm{R} 1=0.132, \mathrm{wR} 2=0.339$ 
Table S8. Atomic coordinates and equivalent isotropic displacement parameters $\left(\AA^{2}\right)$ for QPPOMe/F $\mathbf{F}_{2} \mathbf{T C N Q}$. $\mathrm{U}_{\mathrm{eq}}$ is defined as one third of the trace of the orthogonalized $\mathrm{U}_{\mathrm{ij}}$ tensor.

\begin{tabular}{|c|c|c|c|c|}
\hline Atom & $x$ & $y$ & $z$ & $U_{\text {eq }}$ \\
\hline C11 & $-0.0555(11)$ & $0.6059(9)$ & $0.2294(6)$ & $0.084(3)$ \\
\hline C12 & $-0.0031(9)$ & $0.5972(8)$ & $0.3121(6)$ & $0.066(3)$ \\
\hline N13 & $0.0768(8)$ & $0.5091(6)$ & $0.3379(5)$ & $0.072(2)$ \\
\hline C14 & $0.1207(9)$ & $0.5062(7)$ & $0.4151(6)$ & $0.064(3)$ \\
\hline C15 & $0.2060(9)$ & $0.4121(7)$ & $0.4458(5)$ & $0.059(3)$ \\
\hline C16 & $0.2408(9)$ & $0.3326(7)$ & $0.3971(6)$ & $0.066(3)$ \\
\hline $\mathrm{H} 16$ & 0.2069 & 0.3380 & 0.3428 & 0.080 \\
\hline C17 & $0.3225(9)$ & $0.2452(7)$ & $0.4227(6)$ & $0.067(3)$ \\
\hline C18 & $0.3675(9)$ & $0.2407(7)$ & $0.5047(5)$ & $0.060(3)$ \\
\hline $\mathrm{H} 18$ & 0.4225 & 0.1814 & 0.5255 & 0.072 \\
\hline C19 & $0.3350(9)$ & $0.3188(7)$ & $0.5559(6)$ & $0.057(2)$ \\
\hline C2O & $0.3857(8)$ & $0.3109(7)$ & $0.6389(5)$ & $0.056(2)$ \\
\hline N21 & $0.4662(7)$ & $0.2265(5)$ & $0.6639(4)$ & $0.057(2)$ \\
\hline $\mathrm{C} 22$ & $0.5120(8)$ & $0.2227(7)$ & $0.7424(6)$ & $0.055(2)$ \\
\hline $\mathrm{C} 23$ & $0.5996(8)$ & $0.1338(6)$ & $0.7704(5)$ & $0.055(3)$ \\
\hline $\mathrm{H} 23$ & 0.6231 & 0.0795 & 0.7360 & 0.066 \\
\hline C24 & $0.6483(8)$ & $0.1299(6)$ & $0.8485(5)$ & $0.053(2)$ \\
\hline $\mathrm{C} 25$ & $0.7422(8)$ & $0.0417(7)$ & $0.8881(6)$ & $0.058(2)$ \\
\hline $\mathrm{H} 25$ & 0.7653 & -0.0149 & 0.8510 & 0.069 \\
\hline $\mathrm{C} 26$ & $0.6871(8)$ & $0.0172(7)$ & $0.9716(6)$ & $0.057(2)$ \\
\hline $\mathrm{C} 27$ & $0.6673(8)$ & $-0.0697(7)$ & $1.0007(6)$ & $0.062(3)$ \\
\hline $\mathrm{H} 27$ & 0.6849 & -0.1229 & 0.9649 & 0.074 \\
\hline $\mathrm{C} 28$ & $0.6231(9)$ & $-0.0821(7)$ & $1.0798(6)$ & $0.066(3)$ \\
\hline $\mathrm{H} 28$ & 0.6124 & -0.1434 & 1.0993 & 0.079 \\
\hline C29 & $0.5944(9)$ & $-0.0041(8)$ & $1.1308(6)$ & $0.070(3)$ \\
\hline $\mathrm{H} 29$ & 0.5631 & -0.0127 & 1.1855 & 0.084 \\
\hline C30 & $0.6096(9)$ & $0.0883(8)$ & $1.1047(6)$ & $0.070(3)$ \\
\hline $\mathrm{H} 30$ & 0.5895 & 0.1416 & 1.1404 & 0.084 \\
\hline C31 & $0.6555(8)$ & $0.0971(7)$ & $1.0243(6)$ & $0.058(2)$ \\
\hline C32 & $0.8450(9)$ & $0.0770(7)$ & $0.9093(6)$ & $0.059(2)$ \\
\hline $\mathrm{C} 33$ & $0.9632(9)$ & $0.0419(8)$ & $0.8823(6)$ & $0.064(3)$ \\
\hline H33 & 0.9856 & -0.0108 & 0.8460 & 0.077 \\
\hline C34 & $1.0482(10)$ & $0.0845(8)$ & $0.9088(6)$ & $0.073(3)$ \\
\hline H34 & 1.1297 & 0.0577 & 0.8927 & 0.088 \\
\hline C35 & $1.0184(11)$ & $0.1648(8)$ & $0.9577(6)$ & $0.075(3)$ \\
\hline H35 & 1.0772 & 0.1945 & 0.9737 & 0.090 \\
\hline C36 & $0.8998(10)$ & $0.2001(8)$ & $0.9825(6)$ & $0.068(3)$ \\
\hline H36 & 0.8778 & 0.2545 & 1.0170 & 0.082 \\
\hline $\mathrm{C} 37$ & $0.8138(9)$ & $0.1600(7)$ & $0.9592(6)$ & $0.059(2)$ \\
\hline C38 & $0.6825(8)$ & $0.1887(7)$ & $0.9833(5)$ & $0.058(2)$ \\
\hline H38 & 0.6598 & 0.2456 & 1.0200 & 0.069 \\
\hline C39 & $0.6145(8)$ & $0.2093(7)$ & $0.9004(5)$ & $0.055(2)$ \\
\hline C40 & $0.5311(8)$ & $0.2957(7)$ & $0.8756(5)$ & $0.059(3)$ \\
\hline $\mathrm{H} 40$ & 0.5092 & 0.3490 & 0.9113 & 0.070 \\
\hline C41 & $0.4784(8)$ & $0.3029(7)$ & $0.7945(6)$ & $0.057(2)$ \\
\hline N42 & $0.3959(7)$ & $0.3893(5)$ & $0.7687(4)$ & $0.059(2)$ \\
\hline $\mathrm{C} 43$ & $0.3529(8)$ & $0.3910(7)$ & $0.6901(6)$ & $0.054(2)$ \\
\hline C44 & $0.2686(9)$ & $0.4853(7)$ & $0.6595(6)$ & $0.060(3)$ \\
\hline C45 & $0.2366(9)$ & $0.5650(7)$ & $0.7083(6)$ & $0.069(3)$ \\
\hline H45 & 0.2702 & 0.5596 & 0.7627 & 0.083 \\
\hline C46 & $0.1558(9)$ & $0.6541(7)$ & $0.6803(6)$ & $0.066(3)$ \\
\hline
\end{tabular}




\begin{tabular}{|c|c|c|c|c|}
\hline C47 & $0.1089(9)$ & $0.6578(7)$ & $0.6018(6)$ & $0.062(3)$ \\
\hline $\mathrm{H} 47$ & 0.0529 & 0.7168 & 0.5817 & 0.075 \\
\hline C48 & $0.1392(8)$ & $0.5800(7)$ & $0.5507(6)$ & $0.061(3)$ \\
\hline C49 & $0.0873(8)$ & $0.5852(7)$ & $0.4662(6)$ & $0.059(3)$ \\
\hline N50 & $0.0128(7)$ & $0.6726(6)$ & $0.4401(5)$ & $0.065(2)$ \\
\hline C51 & $-0.0326(9)$ & $0.6765(8)$ & $0.3623(6)$ & $0.066(3)$ \\
\hline C52 & $-0.1163(10)$ & $0.7683(9)$ & $0.3324(7)$ & $0.080(3)$ \\
\hline C53 & $-0.1623(10)$ & $0.7715(9)$ & $0.2535(7)$ & $0.083(3)$ \\
\hline H53 & -0.2172 & 0.8306 & 0.2325 & 0.099 \\
\hline C54 & $-0.1320(10)$ & $0.6922(9)$ & $0.2033(7)$ & $0.084(3)$ \\
\hline H54 & -0.1662 & 0.6989 & 0.1490 & 0.101 \\
\hline C55 & $0.2528(8)$ & $0.4074(7)$ & $0.5263(6)$ & $0.057(2)$ \\
\hline C56 & $0.2204(8)$ & $0.4883(7)$ & $0.5792(5)$ & $0.053(2)$ \\
\hline C57 & $0.3636(12)$ & $0.1573(8)$ & $0.3696(6)$ & $0.089(4)$ \\
\hline C571 & $0.3273(13)$ & $0.1772(9)$ & $0.2795(6)$ & $0.122(5)$ \\
\hline H57A & 0.3491 & 0.2352 & 0.2564 & 0.182 \\
\hline H57B & 0.3685 & 0.1206 & 0.2474 & 0.182 \\
\hline H57C & 0.2403 & 0.1887 & 0.2755 & 0.182 \\
\hline C572 & $0.5004(15)$ & $0.1266(14)$ & $0.3626(10)$ & $0.181(7)$ \\
\hline H57D & 0.5269 & 0.1803 & 0.3348 & 0.272 \\
\hline H57E & 0.5349 & 0.1114 & 0.4189 & 0.272 \\
\hline H57F & 0.5272 & 0.0686 & 0.3294 & 0.272 \\
\hline C573 & $0.337(3)$ & $0.0737(11)$ & $0.4106(9)$ & $0.212(9)$ \\
\hline H57G & 0.3633 & 0.0661 & 0.4691 & 0.318 \\
\hline $\mathrm{H} 57 \mathrm{H}$ & 0.2498 & 0.0830 & 0.4095 & 0.318 \\
\hline H57I & 0.3780 & 0.0149 & 0.3814 & 0.318 \\
\hline C58 & $0.1218(11)$ & $0.7403(7)$ & $0.7358(7)$ & $0.081(3)$ \\
\hline C581 & $0.075(2)$ & $0.8367(10)$ & $0.6835(9)$ & $0.216(10)$ \\
\hline H58A & 0.0094 & 0.8326 & 0.6479 & 0.324 \\
\hline H58B & 0.0456 & 0.8904 & 0.7211 & 0.324 \\
\hline H58C & 0.1401 & 0.8488 & 0.6480 & 0.324 \\
\hline C582 & $0.0248(13)$ & $0.7260(12)$ & $0.7982(9)$ & $0.135(5)$ \\
\hline H58D & -0.0449 & 0.7216 & 0.7673 & 0.202 \\
\hline H58E & 0.0566 & 0.6655 & 0.8332 & 0.202 \\
\hline H58F & 0.0009 & 0.7818 & 0.8342 & 0.202 \\
\hline C583 & $0.2226(14)$ & $0.7459(11)$ & $0.7907(11)$ & $0.143(6)$ \\
\hline H58G & 0.1953 & 0.8030 & 0.8249 & 0.214 \\
\hline $\mathrm{H} 58 \mathrm{H}$ & 0.2476 & 0.6862 & 0.8275 & 0.214 \\
\hline H58I & 0.2906 & 0.7523 & 0.7552 & 0.214 \\
\hline O59 & $-0.0182(8)$ & $0.5254(6)$ & $0.1841(5)$ & $0.103(3)$ \\
\hline C59 & $-0.0697(15)$ & $0.5299(10)$ & $0.1026(7)$ & $0.137(6)$ \\
\hline H59A & -0.0355 & 0.4675 & 0.0764 & 0.205 \\
\hline H59B & -0.1568 & 0.5423 & 0.1085 & 0.205 \\
\hline H59C & -0.0521 & 0.5830 & 0.0672 & 0.205 \\
\hline $\mathrm{O} 60$ & $-0.1398(7)$ & $0.8403(6)$ & $0.3855(5)$ & $0.092(3)$ \\
\hline C60 & $-0.2218(12)$ & $0.9350(8)$ & $0.3576(8)$ & $0.114(5)$ \\
\hline $\mathrm{H} 60 \mathrm{~A}$ & -0.2312 & 0.9809 & 0.4023 & 0.171 \\
\hline H60B & -0.1890 & 0.9610 & 0.3069 & 0.171 \\
\hline $\mathrm{H} 60 \mathrm{C}$ & -0.3001 & 0.9264 & 0.3451 & 0.171 \\
\hline C61 & $0.1188(15)$ & $0.8760(16)$ & $0.4289(8)$ & $0.125(11)$ \\
\hline H61 & 0.0847 & 0.8201 & 0.4436 & 0.150 \\
\hline $\mathrm{Cl} 1$ & $0.2577(7)$ & $0.8385(6)$ & $0.4657(5)$ & $0.122(3)$ \\
\hline $\mathrm{Cl} 2$ & $0.1110(7)$ & $0.8904(7)$ & $0.3228(4)$ & $0.142(3)$ \\
\hline $\mathrm{Cl} 3$ & $0.0267(9)$ & $0.9715(7)$ & $0.4783(6)$ & $0.149(4)$ \\
\hline C62 & $0.661(4)$ & $0.5541(19)$ & $0.9196(12)$ & $0.34(5)$ \\
\hline H62 & 0.5736 & 0.5876 & 0.9109 & 0.414 \\
\hline $\mathrm{Cl} 4$ & $0.6589(18)$ & $0.4788(10)$ & $1.0045(11)$ & $0.199(8)$ \\
\hline $\mathrm{Cl} 5$ & $0.7181(16)$ & $0.6481(10)$ & $0.9269(10)$ & $0.221(10)$ \\
\hline Cl6 & $0.701(6)$ & $0.499(4)$ & $0.830(2)$ & $0.73(5)$ \\
\hline C71 & $0.244(3)$ & $0.685(2)$ & $0.294(2)$ & $0.167(11)$ \\
\hline C72 & $0.371(2)$ & $0.572(2)$ & $0.3701(18)$ & $0.128(9)$ \\
\hline
\end{tabular}




$\begin{array}{lllll}\text { C73 } & 0.428(4) & 0.549(3) & 0.434(3) & 0.232(17) \\ \text { C74 } & 0.445(3) & 0.621(2) & 0.5750(19) & 0.164(11) \\ \text { C75 } & 0.688(3) & 0.349(2) & 0.6298(18) & 0.141(9) \\ \text { C76 } & 0.773(3) & 0.346(2) & 0.782(2) & 0.161(11) \\ \text { C77 } & 0.7541(19) & 0.4462(15) & 0.9056(16) & 0.088(6) \\ \text { C78 } & 0.579(2) & 0.5814(17) & 0.8130(17) & 0.083(7) \\ \text { C79 } & 0.393(2) & 0.506(2) & 0.3080(18) & 0.140(9) \\ \text { C80 } & 0.697(4) & 0.272(4) & 0.543(3) & 0.243(18) \\ \text { C81 } & 0.767(5) & 0.445(3) & 0.964(3) & 0.213(18) \\ \text { C82 } & 0.533(3) & 0.663(3) & 0.858(2) & 0.191(14) \\ \text { C83 } & 0.558(3) & 0.582(3) & 0.852(2) & 0.153(15) \\ \text { C84 } & 0.677(4) & 0.440(3) & 0.773(3) & 0.234(17) \\ & & & & \end{array}$

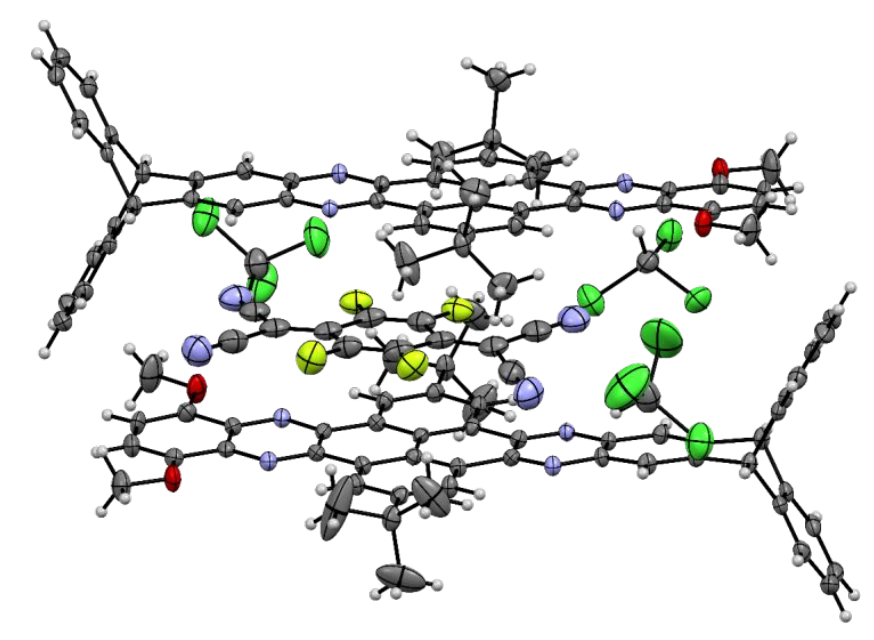

Figure S16. Thermal atomic displacement ellipsoid plot of the asymmetric unit of QPP-OMe/F ${ }_{4} \mathbf{T C N Q}$. The ellipsoids of non-hydrogen atoms are drawn at the $50 \%$ probability level and hydrogen atoms are represented by a sphere of arbitrary size.

Table S9. Crystal data and structure refinement for $\mathrm{QPP}-\mathrm{OMe} / \mathrm{F}_{4} \mathrm{TCNQ}$.

CCDC
Solvent
Empirical formula
Formula weight
Temperature
Wavelength
Crystal system
Space group
Z
Unit cell dimensions

Volume
Density (calculated)
Absorption coefficient
Crystal shape
Crystal size
Crystal colour
Theta range for data collection
Index ranges
Reflections collected

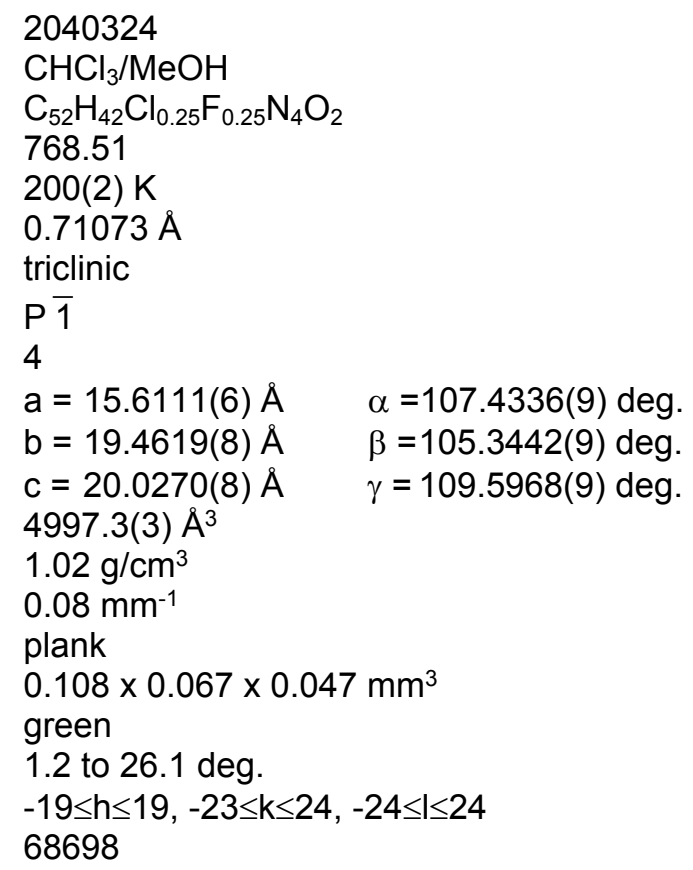


Independent reflections

Observed reflections

Absorption correction

Max. and min. transmission

Refinement method

Data/restraints/parameters

Goodness-of-fit on $\mathrm{F}^{2}$

Final $R$ indices ( $\mid>2$ sigma(I))

Largest diff. peak and hole
$19729(\mathrm{R}$ (int) $=0.0601)$

$10973(\mathrm{I}>2 \sigma(\mathrm{I}))$

Semi-empirical from equivalents

0.96 and 0.92

Full-matrix least-squares on $\mathrm{F}^{2}$

19729 / 0 / 1349

1.03

$\mathrm{R} 1=0.066, \mathrm{wR} 2=0.157$

1.45 and -0.93 e $\AA^{-3}$

Table S10. Atomic coordinates and equivalent isotropic displacement parameters $\left(\AA^{2}\right)$ for QPPOMe/ $F_{4} T C N Q . U_{\text {eq }}$ is defined as one third of the trace of the orthogonalized $U_{i j}$ tensor.

\begin{tabular}{|c|c|c|c|c|}
\hline Atom & $x$ & $y$ & $z$ & $U_{\text {eq }}$ \\
\hline C1 & $0.4569(3)$ & $0.7768(2)$ & $0.6504(2)$ & $0.0456(11)$ \\
\hline $\mathrm{C} 2$ & $0.2781(3)$ & $0.6892(2)$ & $0.8436(2)$ & $0.0433(10)$ \\
\hline C11 & $0.4127(3)$ & $0.7529(2)$ & $0.6959(2)$ & $0.0410(10)$ \\
\hline C12 & $0.4623(3)$ & $0.7394(2)$ & $0.7591(2)$ & $0.0413(10)$ \\
\hline F12 & $0.5555(2)$ & $0.7505(1)$ & $0.7733(1)$ & $0.0531(6)$ \\
\hline C13 & $0.4200(3)$ & $0.7195(2)$ & $0.8049(2)$ & $0.0416(10)$ \\
\hline F13 & $0.4730(2)$ & $0.7107(1)$ & $0.8636(1)$ & $0.0515(6)$ \\
\hline C14 & $0.3210(3)$ & $0.7082(2)$ & $0.7958(2)$ & $0.0392(10)$ \\
\hline C15 & $0.2708(3)$ & $0.7187(2)$ & $0.7307(2)$ & $0.0445(10)$ \\
\hline F15 & $0.1765(2)$ & $0.7059(2)$ & $0.7149(2)$ & $0.0631(7)$ \\
\hline C16 & $0.3138(3)$ & $0.7395(2)$ & $0.6855(2)$ & $0.0449(10)$ \\
\hline F16 & $0.2591(2)$ & $0.7455(2)$ & $0.6257(1)$ & $0.0592(7)$ \\
\hline C17 & $0.5527(4)$ & $0.7843(2)$ & $0.6545(3)$ & $0.0498(11)$ \\
\hline N17 & $0.6265(3)$ & $0.7899(2)$ & $0.6511(2)$ & $0.0659(12)$ \\
\hline C18 & $0.4126(3)$ & $0.8019(3)$ & $0.5952(3)$ & $0.0491(11)$ \\
\hline N18 & $0.3829(3)$ & $0.8245(3)$ & $0.5525(2)$ & $0.0659(12)$ \\
\hline C19 & $0.1790(4)$ & $0.6788(3)$ & $0.8345(3)$ & $0.0497(11)$ \\
\hline N19 & $0.1013(3)$ & $0.6686(3)$ & $0.8316(2)$ & $0.0672(12)$ \\
\hline C20 & $0.3253(3)$ & $0.6771(2)$ & $0.9074(3)$ & $0.0459(11)$ \\
\hline N20 & $0.3583(3)$ & $0.6663(2)$ & $0.9597(2)$ & $0.0587(10)$ \\
\hline C3 & $0.5542(3)$ & $0.6722(3)$ & $1.0706(2)$ & $0.0511(11)$ \\
\hline H3 & 0.5185 & 0.7058 & 1.0669 & 0.061 \\
\hline $\mathrm{Cl} 1$ & $0.4764(1)$ & $0.5841(1)$ & $1.0726(1)$ & $0.0707(4)$ \\
\hline $\mathrm{Cl} 2$ & $0.6626(1)$ & $0.7273(1)$ & $1.1562(1)$ & $0.0781(4)$ \\
\hline $\mathrm{Cl} 3$ & $0.5832(1)$ & $0.6478(1)$ & $0.9912(1)$ & $0.0862(5)$ \\
\hline C4 & $0.8807(3)$ & $0.7828(2)$ & $0.7187(2)$ & $0.0396(10)$ \\
\hline $\mathrm{H} 4$ & 0.8284 & 0.7272 & 0.6808 & 0.047 \\
\hline $\mathrm{Cl} 4$ & $0.8377(1)$ & $0.8201(1)$ & $0.7870(1)$ & $0.0613(3)$ \\
\hline $\mathrm{Cl} 5$ & $0.9011(1)$ & $0.8439(1)$ & $0.6694(1)$ & $0.0543(3)$ \\
\hline $\mathrm{Cl} 6$ & $0.9891(1)$ & $0.7765(1)$ & $0.7626(1)$ & $0.0598(3)$ \\
\hline C5 & $0.4540(3)$ & $0.8555(3)$ & $0.4254(3)$ & $0.0618(13)$ \\
\hline H5 & 0.4510 & 0.8750 & 0.4765 & 0.074 \\
\hline $\mathrm{Cl} 7$ & $0.5260(1)$ & $0.9400(1)$ & $0.4156(1)$ & $0.0702(4)$ \\
\hline $\mathrm{Cl} 8$ & $0.5046(2)$ & $0.7905(1)$ & $0.4225(2)$ & $0.1435(9)$ \\
\hline Cl9 & $0.3331(1)$ & $0.8095(1)$ & $0.3554(1)$ & $0.1237(7)$ \\
\hline C111 & $0.2444(3)$ & $0.8506(2)$ & $1.0192(2)$ & $0.0277(8)$ \\
\hline C121 & $0.3325(2)$ & $0.8652(2)$ & $1.0047(2)$ & $0.0236(7)$ \\
\hline N131 & $0.3384(2)$ & $0.8867(2)$ & $0.9479(2)$ & $0.0242(6)$ \\
\hline C141 & $0.4215(2)$ & $0.9004(2)$ & $0.9362(2)$ & $0.0216(7)$ \\
\hline C151 & $0.4277(2)$ & $0.9224(2)$ & $0.8729(2)$ & $0.0216(7)$ \\
\hline C161 & $0.3485(2)$ & $0.9274(2)$ & $0.8263(2)$ & $0.0259(8)$ \\
\hline H161 & 0.2904 & 0.9173 & 0.8363 & 0.031 \\
\hline C171 & $0.3522(2)$ & $0.9470(2)$ & $0.7653(2)$ & $0.0243(7)$ \\
\hline
\end{tabular}




\begin{tabular}{|c|c|c|c|c|}
\hline C181 & $0.4379(2)$ & $0.9608(2)$ & $0.7521(2)$ & $0.0244(8)$ \\
\hline H181 & 0.4415 & 0.9740 & 0.7107 & 0.029 \\
\hline C191 & $0.5188(2)$ & $0.9560(2)$ & $0.7970(2)$ & $0.0210(7)$ \\
\hline C201 & $0.6078(2)$ & $0.9696(2)$ & $0.7811(2)$ & $0.0210(7)$ \\
\hline N211 & $0.6087(2)$ & $0.9841(2)$ & $0.7203(2)$ & $0.0223(6)$ \\
\hline C221 & $0.6921(2)$ & $0.9975(2)$ & $0.7072(2)$ & $0.0208(7)$ \\
\hline C231 & $0.6968(2)$ & $1.0116(2)$ & $0.6423(2)$ & $0.0239(7)$ \\
\hline H231 & 0.6404 & 1.0093 & 0.6070 & 0.029 \\
\hline C241 & $0.7818(2)$ & $1.0283(2)$ & $0.6307(2)$ & $0.0217(7)$ \\
\hline C251 & $0.8007(2)$ & $1.0453(2)$ & $0.5651(2)$ & $0.0233(7)$ \\
\hline H251 & 0.7413 & 1.0428 & 0.5279 & 0.028 \\
\hline C261 & $0.8340(2)$ & $0.9844(2)$ & $0.5282(2)$ & $0.0232(7)$ \\
\hline C271 & $0.7915(3)$ & $0.9296(2)$ & $0.4529(2)$ & $0.0306(8)$ \\
\hline H271 & 0.7334 & 0.9255 & 0.4179 & 0.037 \\
\hline C281 & $0.8338(3)$ & $0.8803(2)$ & $0.4284(2)$ & $0.0378(9)$ \\
\hline H281 & 0.8053 & 0.8431 & 0.3761 & 0.045 \\
\hline C291 & $0.9175(3)$ & $0.8847(2)$ & $0.4793(2)$ & $0.0365(9)$ \\
\hline H291 & 0.9456 & 0.8502 & 0.4618 & 0.044 \\
\hline C301 & $0.9603(3)$ & $0.9392(2)$ & $0.5556(2)$ & $0.0281(8)$ \\
\hline H301 & 1.0175 & 0.9421 & 0.5906 & 0.034 \\
\hline C311 & $0.9192(2)$ & $0.9893(2)$ & $0.5804(2)$ & $0.0243(8)$ \\
\hline C321 & $0.8906(2)$ & $1.1278(2)$ & $0.6029(2)$ & $0.0237(7)$ \\
\hline C331 & $0.8962(3)$ & $1.1947(2)$ & $0.5891(2)$ & $0.0299(8)$ \\
\hline H331 & 0.8398 & 1.1921 & 0.5536 & 0.036 \\
\hline C341 & $0.9849(3)$ & $1.2651(2)$ & $0.6277(2)$ & $0.0359(9)$ \\
\hline H341 & 0.9895 & 1.3107 & 0.6178 & 0.043 \\
\hline C351 & $1.0673(3)$ & $1.2697(2)$ & $0.6809(2)$ & $0.0346(9)$ \\
\hline H351 & 1.1277 & 1.3184 & 0.7076 & 0.042 \\
\hline C361 & $1.0611(3)$ & $1.2029(2)$ & $0.6949(2)$ & $0.0295(8)$ \\
\hline H361 & 1.1169 & 1.2062 & 0.7318 & 0.035 \\
\hline C371 & $0.9740(2)$ & $1.1318(2)$ & $0.6555(2)$ & $0.0235(7)$ \\
\hline C381 & $0.9560(2)$ & $1.0528(2)$ & $0.6614(2)$ & $0.0230(7)$ \\
\hline H381 & 1.0161 & 1.0557 & 0.6983 & 0.028 \\
\hline C391 & $0.8674(2)$ & $1.0314(2)$ & $0.6834(2)$ & $0.0205(7)$ \\
\hline C401 & $0.8645(2)$ & $1.0159(2)$ & $0.7446(2)$ & $0.0225(7)$ \\
\hline H401 & 0.9211 & 1.0168 & 0.7783 & 0.027 \\
\hline C411 & $0.7761(2)$ & $0.9983(2)$ & $0.7582(2)$ & $0.0194(7)$ \\
\hline N421 & $0.7741(2)$ & $0.9823(2)$ & $0.8191(1)$ & $0.0205(6)$ \\
\hline C431 & $0.6905(2)$ & $0.9668(2)$ & $0.8300(2)$ & $0.0203(7)$ \\
\hline C441 & $0.6838(2)$ & $0.9447(2)$ & $0.8930(2)$ & $0.0205(7)$ \\
\hline C451 & $0.7607(2)$ & $0.9363(2)$ & $0.9379(2)$ & $0.0232(7)$ \\
\hline H451 & 0.8187 & 0.9461 & 0.9278 & 0.028 \\
\hline C461 & $0.7560(2)$ & $0.9140(2)$ & $0.9972(2)$ & $0.0239(7)$ \\
\hline C471 & $0.6708(2)$ & $0.9004(2)$ & $1.0112(2)$ & $0.0243(8)$ \\
\hline H471 & 0.6664 & 0.8856 & 1.0517 & 0.029 \\
\hline C481 & $0.5912(2)$ & $0.9080(2)$ & $0.9674(2)$ & $0.0204(7)$ \\
\hline C491 & $0.5015(2)$ & $0.8923(2)$ & $0.9819(2)$ & $0.0220(7)$ \\
\hline N501 & $0.4954(2)$ & $0.8694(2)$ & $1.0375(2)$ & $0.0248(6)$ \\
\hline C511 & $0.4117(3)$ & $0.8558(2)$ & $1.0501(2)$ & $0.0247(8)$ \\
\hline C521 & $0.4003(3)$ & $0.8304(2)$ & $1.1085(2)$ & $0.0294(8)$ \\
\hline C531 & $0.3170(3)$ & $0.8194(2)$ & $1.1221(2)$ & $0.0338(9)$ \\
\hline H531 & 0.3108 & 0.8046 & 1.1623 & 0.041 \\
\hline C541 & $0.2394(3)$ & $0.8294(2)$ & $1.0776(2)$ & $0.0337(9)$ \\
\hline H541 & 0.1821 & 0.8213 & 1.0886 & 0.040 \\
\hline C551 & $0.5150(2)$ & $0.9368(2)$ & $0.8597(2)$ & $0.0202(7)$ \\
\hline C561 & $0.5969(2)$ & $0.9301(2)$ & $0.9069(2)$ & $0.0192(7)$ \\
\hline C571 & $0.2653(2)$ & $0.9546(2)$ & $0.7160(2)$ & $0.0308(8)$ \\
\hline C5711 & $0.1709(3)$ & $0.9122(5)$ & $0.7224(4)$ & $0.136(4)$ \\
\hline H57A1 & 0.1179 & 0.9199 & 0.6912 & 0.204 \\
\hline H57B1 & 0.1801 & 0.9344 & 0.7760 & 0.204 \\
\hline H57C1 & 0.1525 & 0.8545 & 0.7040 & 0.204 \\
\hline
\end{tabular}




\begin{tabular}{|c|c|c|c|c|}
\hline C5721 & $0.2914(4)$ & $1.0441(3)$ & $0.7427(3)$ & $0.089(2)$ \\
\hline H57D1 & 0.2366 & 1.0501 & 0.7116 & 0.133 \\
\hline H57E1 & 0.3521 & 1.0722 & 0.7370 & 0.133 \\
\hline H57F1 & 0.3021 & 1.0674 & 0.7966 & 0.133 \\
\hline C5731 & $0.2482(4)$ & $0.9234(4)$ & $0.6328(3)$ & $0.0811(18)$ \\
\hline H57G1 & 0.1933 & 0.9309 & 0.6039 & 0.122 \\
\hline $\mathrm{H} 57 \mathrm{H} 1$ & 0.2313 & 0.8659 & 0.6126 & 0.122 \\
\hline H57I1 & 0.3087 & 0.9532 & 0.6277 & 0.122 \\
\hline C581 & $0.8425(3)$ & $0.9031(2)$ & $1.0421(2)$ & $0.0314(9)$ \\
\hline C5811 & $0.8269(3)$ & $0.8803(3)$ & $1.1065(2)$ & $0.0501(12)$ \\
\hline H58A1 & 0.8836 & 0.8730 & 1.1326 & 0.075 \\
\hline H58B1 & 0.7657 & 0.8299 & 1.0846 & 0.075 \\
\hline $\mathrm{H} 58 \mathrm{C} 1$ & 0.8216 & 0.9233 & 1.1432 & 0.075 \\
\hline C5821 & $0.8513(4)$ & $0.8351(3)$ & $0.9870(3)$ & $0.0627(14)$ \\
\hline H58D1 & 0.9048 & 0.8258 & 1.0152 & 0.094 \\
\hline H58E1 & 0.8666 & 0.8499 & 0.9474 & 0.094 \\
\hline H58F1 & 0.7884 & 0.7857 & 0.9629 & 0.094 \\
\hline C5831 & $0.9380(3)$ & $0.9808(3)$ & $1.0770(3)$ & $0.0714(16)$ \\
\hline H58G1 & 0.9950 & 0.9706 & 1.0960 & 0.107 \\
\hline $\mathrm{H} 58 \mathrm{H} 1$ & 0.9371 & 1.0214 & 1.1196 & 0.107 \\
\hline H58I1 & 0.9437 & 1.0006 & 1.0380 & 0.107 \\
\hline O591 & $0.1738(2)$ & $0.8601(2)$ & $0.9721(1)$ & $0.0363(6)$ \\
\hline C591 & $0.0833(3)$ & $0.8428(3)$ & $0.9826(2)$ & $0.0452(11)$ \\
\hline H59A1 & 0.0365 & 0.8486 & 0.9437 & 0.068 \\
\hline H59B1 & 0.0971 & 0.8806 & 1.0337 & 0.068 \\
\hline H59C1 & 0.0543 & 0.7875 & 0.9777 & 0.068 \\
\hline O601 & $0.4768(2)$ & $0.8182(2)$ & $1.1461(2)$ & $0.0418(7)$ \\
\hline C601 & $0.4662(4)$ & $0.7916(3)$ & $1.2037(3)$ & $0.0632(14)$ \\
\hline H60A1 & 0.5242 & 0.7838 & 1.2263 & 0.095 \\
\hline H60B1 & 0.4057 & 0.7404 & 1.1808 & 0.095 \\
\hline $\mathrm{H} 60 \mathrm{C} 1$ & 0.4615 & 0.8322 & 1.2437 & 0.095 \\
\hline C112 & $0.6226(3)$ & $0.6508(2)$ & $0.3947(2)$ & $0.0281(8)$ \\
\hline C122 & $0.6127(2)$ & $0.6354(2)$ & $0.4588(2)$ & $0.0238(7)$ \\
\hline N132 & $0.5254(2)$ & $0.6184(2)$ & $0.4661(2)$ & $0.0236(6)$ \\
\hline C142 & $0.5193(2)$ & $0.6032(2)$ & $0.5253(2)$ & $0.0221(7)$ \\
\hline C152 & $0.4257(2)$ & $0.5855(2)$ & $0.5354(2)$ & $0.0216(7)$ \\
\hline C162 & $0.3446(2)$ & $0.5842(2)$ & $0.4835(2)$ & $0.0249(8)$ \\
\hline H162 & 0.3509 & 0.5956 & 0.4417 & 0.030 \\
\hline C172 & $0.2541(2)$ & $0.5665(2)$ & $0.4915(2)$ & $0.0244(8)$ \\
\hline C182 & $0.2474(2)$ & $0.5506(2)$ & $0.5533(2)$ & $0.0231(7)$ \\
\hline H182 & 0.1866 & 0.5387 & 0.5596 & 0.028 \\
\hline C192 & $0.3267(2)$ & $0.5516(2)$ & $0.6068(2)$ & $0.0203(7)$ \\
\hline C202 & $0.3168(2)$ & $0.5330(2)$ & $0.6706(2)$ & $0.0187(7)$ \\
\hline N212 & $0.2314(2)$ & $0.5180(2)$ & $0.6789(1)$ & $0.0200(6)$ \\
\hline C222 & $0.2240(2)$ & $0.4995(2)$ & $0.7380(2)$ & $0.0191(7)$ \\
\hline C232 & $0.1358(2)$ & $0.4850(2)$ & $0.7512(2)$ & $0.0207(7)$ \\
\hline H232 & 0.0818 & 0.4876 & 0.7182 & 0.025 \\
\hline C242 & $0.1290(2)$ & $0.4676(2)$ & $0.8108(2)$ & $0.0199(7)$ \\
\hline C252 & $0.0402(2)$ & $0.4500(2)$ & $0.8326(2)$ & $0.0238(7)$ \\
\hline H252 & -0.0168 & 0.4521 & 0.7974 & 0.029 \\
\hline C262 & $0.0802(2)$ & $0.5111(2)$ & $0.9154(2)$ & $0.0230(7)$ \\
\hline C272 & $0.0446(3)$ & $0.5645(2)$ & $0.9435(2)$ & $0.0291(8)$ \\
\hline $\mathrm{H} 272$ & -0.0090 & 0.5668 & 0.9098 & 0.035 \\
\hline $\mathrm{C} 282$ & $0.0876(3)$ & $0.6146(2)$ & $1.0211(2)$ & $0.0346(9)$ \\
\hline H282 & 0.0626 & 0.6507 & 1.0409 & 0.042 \\
\hline C292 & $0.1669(3)$ & $0.6122(2)$ & $1.0699(2)$ & $0.0331(9)$ \\
\hline H292 & 0.1961 & 0.6467 & 1.1231 & 0.040 \\
\hline C302 & $0.2042(3)$ & $0.5596(2)$ & $1.0417(2)$ & $0.0272(8)$ \\
\hline H302 & 0.2599 & 0.5592 & 1.0751 & 0.033 \\
\hline C312 & $0.1599(2)$ & $0.5079(2)$ & $0.9648(2)$ & $0.0223(7)$ \\
\hline C322 & $0.0130(2)$ & $0.3680(2)$ & $0.8343(2)$ & $0.0213(7)$ \\
\hline
\end{tabular}




\begin{tabular}{|c|c|c|c|c|}
\hline C332 & $-0.0785(3)$ & $0.3003(2)$ & $0.7926(2)$ & $0.0300(8)$ \\
\hline H332 & -0.1316 & 0.3017 & 0.7574 & 0.036 \\
\hline C342 & $-0.0919(3)$ & $0.2303(2)$ & $0.8029(2)$ & $0.0316(9)$ \\
\hline H342 & -0.1549 & 0.1840 & 0.7754 & 0.038 \\
\hline C352 & $-0.0142(3)$ & $0.2282(2)$ & $0.8527(2)$ & $0.0323(9)$ \\
\hline H352 & -0.0239 & 0.1803 & 0.8595 & 0.039 \\
\hline C362 & $0.0784(3)$ & $0.2951(2)$ & $0.8933(2)$ & $0.0273(8)$ \\
\hline H362 & 0.1320 & 0.2927 & 0.9270 & 0.033 \\
\hline C372 & $0.0919(2)$ & $0.3647(2)$ & $0.8843(2)$ & $0.0218(7)$ \\
\hline C382 & $0.1869(2)$ & $0.4441(2)$ & $0.9243(2)$ & $0.0202(7)$ \\
\hline H382 & 0.2432 & 0.4417 & 0.9600 & 0.024 \\
\hline C392 & $0.2097(2)$ & $0.4633(2)$ & $0.8608(2)$ & $0.0194(7)$ \\
\hline C402 & $0.2947(2)$ & $0.4763(2)$ & $0.8499(2)$ & $0.0204(7)$ \\
\hline $\mathrm{H} 402$ & 0.3472 & 0.4723 & 0.8829 & 0.024 \\
\hline C412 & $0.3046(2)$ & $0.4961(2)$ & $0.7886(2)$ & $0.0200(7)$ \\
\hline N422 & $0.3915(2)$ & $0.5125(2)$ & $0.7801(1)$ & $0.0212(6)$ \\
\hline C432 & $0.3983(2)$ & $0.5318(2)$ & $0.7229(2)$ & $0.0200(7)$ \\
\hline C442 & $0.4931(2)$ & $0.5527(2)$ & $0.7146(2)$ & $0.0200(7)$ \\
\hline C452 & $0.5736(2)$ & $0.5549(2)$ & $0.7669(2)$ & $0.0236(7)$ \\
\hline H452 & 0.5669 & 0.5439 & 0.8089 & 0.028 \\
\hline C462 & $0.6640(2)$ & $0.5727(2)$ & $0.7598(2)$ & $0.0225(7)$ \\
\hline C472 & $0.6711(2)$ & $0.5884(2)$ & $0.6978(2)$ & $0.0240(8)$ \\
\hline $\mathrm{H} 472$ & 0.7317 & 0.5998 & 0.6913 & 0.029 \\
\hline C482 & $0.5925(2)$ & $0.5881(2)$ & $0.6449(2)$ & $0.0206(7)$ \\
\hline C492 & $0.6015(2)$ & $0.6057(2)$ & $0.5798(2)$ & $0.0202(7)$ \\
\hline N502 & $0.6887(2)$ & $0.6239(2)$ & $0.5738(2)$ & $0.0231(6)$ \\
\hline C512 & $0.6956(2)$ & $0.6389(2)$ & $0.5133(2)$ & $0.0234(7)$ \\
\hline C522 & $0.7874(3)$ & $0.6578(2)$ & $0.5039(2)$ & $0.0269(8)$ \\
\hline C532 & $0.7932(3)$ & $0.6725(2)$ & $0.4427(2)$ & $0.0303(8)$ \\
\hline H532 & 0.8540 & 0.6855 & 0.4365 & 0.036 \\
\hline C542 & $0.7117(3)$ & $0.6690(2)$ & $0.3882(2)$ & $0.0316(9)$ \\
\hline H542 & 0.7186 & 0.6794 & 0.3460 & 0.038 \\
\hline C552 & $0.4178(2)$ & $0.5690(2)$ & $0.5983(2)$ & $0.0197(7)$ \\
\hline C562 & $0.5015(2)$ & $0.5699(2)$ & $0.6523(2)$ & $0.0192(7)$ \\
\hline C572 & $0.1640(2)$ & $0.5595(2)$ & $0.4302(2)$ & $0.0284(8)$ \\
\hline C5712 & $0.1937(3)$ & $0.6273(3)$ & $0.4052(3)$ & $0.0492(11)$ \\
\hline H57A2 & 0.1356 & 0.6194 & 0.3640 & 0.074 \\
\hline H57B2 & 0.2458 & 0.6270 & 0.3866 & 0.074 \\
\hline H57C2 & 0.2190 & 0.6792 & 0.4488 & 0.074 \\
\hline C5722 & $0.1235(3)$ & $0.4789(3)$ & $0.3601(2)$ & $0.0501(11)$ \\
\hline H57D2 & 0.0679 & 0.4735 & 0.3186 & 0.075 \\
\hline H57E2 & 0.1005 & 0.4341 & 0.3742 & 0.075 \\
\hline H57F2 & 0.1765 & 0.4778 & 0.3428 & 0.075 \\
\hline C5732 & $0.0828(3)$ & $0.5598(4)$ & $0.4576(2)$ & $0.0655(15)$ \\
\hline H57G2 & 0.0280 & 0.5573 & 0.4177 & 0.098 \\
\hline $\mathrm{H} 57 \mathrm{H} 2$ & 0.1094 & 0.6093 & 0.5042 & 0.098 \\
\hline H57I2 & 0.0583 & 0.5129 & 0.4686 & 0.098 \\
\hline C582 & $0.7506(2)$ & $0.5712(2)$ & $0.8166(2)$ & $0.0269(8)$ \\
\hline C5812 & $0.7460(3)$ & $0.4878(2)$ & $0.7833(2)$ & $0.0400(10)$ \\
\hline H58A2 & 0.8009 & 0.4857 & 0.8188 & 0.060 \\
\hline H58B2 & 0.7517 & 0.4775 & 0.7341 & 0.060 \\
\hline H58C2 & 0.6824 & 0.4467 & 0.7756 & 0.060 \\
\hline C5822 & $0.7442(3)$ & $0.5873(3)$ & $0.8945(2)$ & $0.0391(10)$ \\
\hline H58D2 & 0.8043 & 0.5921 & 0.9310 & 0.059 \\
\hline H58E2 & 0.6856 & 0.5425 & 0.8888 & 0.059 \\
\hline H58F2 & 0.7386 & 0.6375 & 0.9134 & 0.059 \\
\hline C5832 & $0.8508(3)$ & $0.6369(2)$ & $0.8314(2)$ & $0.0422(10)$ \\
\hline H58G2 & 0.9045 & 0.6373 & 0.8704 & 0.063 \\
\hline $\mathrm{H} 58 \mathrm{H} 2$ & 0.8507 & 0.6896 & 0.8494 & 0.063 \\
\hline H58I2 & 0.8610 & 0.6257 & 0.7838 & 0.063 \\
\hline O592 & $0.5390(2)$ & $0.6452(2)$ & $0.3449(1)$ & $0.0356(6)$ \\
\hline
\end{tabular}




\begin{tabular}{lllll} 
C592 & $0.5473(3)$ & $0.6603(3)$ & $0.2808(2)$ & $0.0471(11)$ \\
H59A2 & 0.4835 & 0.6544 & 0.2485 & 0.071 \\
H59B2 & 0.5651 & 0.6216 & 0.2509 & 0.071 \\
H59C2 & 0.5990 & 0.7152 & 0.2990 & 0.071 \\
O602 & $0.8622(2)$ & $0.6596(2)$ & $0.5592(1)$ & $0.0354(6)$ \\
C602 & $0.9554(3)$ & $0.6816(3)$ & $0.5528(3)$ & $0.0532(12)$ \\
H60A2 & 1.0043 & 0.6833 & 0.5964 & 0.080 \\
H60B2 & 0.9784 & 0.7350 & 0.5525 & 0.080 \\
H60C2 & 0.9476 & 0.6418 & 0.5051 & 0.080 \\
& & & & \\
\hline
\end{tabular}

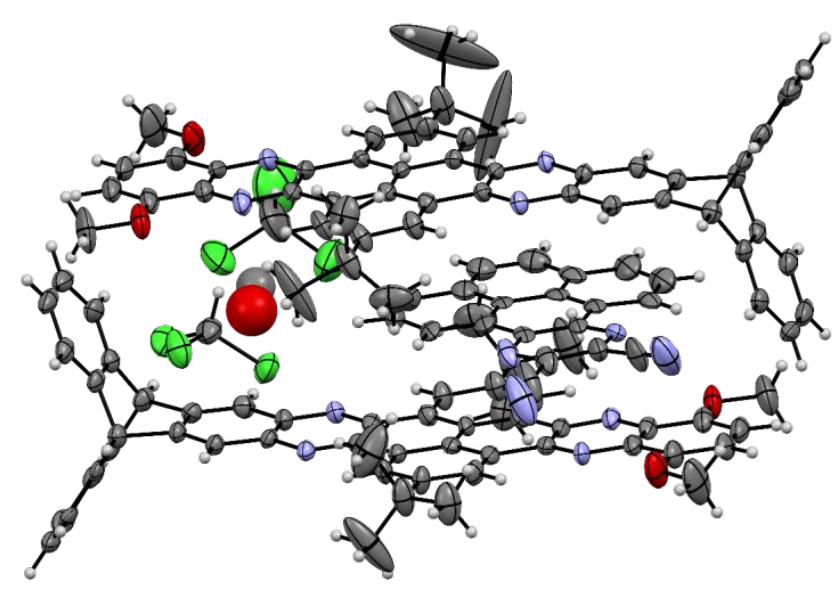

Figure S17. Thermal atomic displacement ellipsoid plot of the asymmetric unit of QPP-OMe/PQDC. The ellipsoids of non-hydrogen atoms are drawn at the $50 \%$ probability level and hydrogen atoms are represented by a sphere of arbitrary size.

Table S11. Crystal data and structure refinement for QPP-OMe/PQDC.

CCDC

Empirical formula

Formula weight

Temperature

Wavelength

Crystal system

Space group

Z

Unit cell dimensions

Volume

Density (calculated)

Absorption coefficient

Crystal shape

Crystal size

Crystal colour

Theta range for data collection

Index ranges

Reflections collected

Independent reflections

Observed reflections

Absorption correction

Max. and min. transmission
2040325

$\mathrm{CHCl}_{3} / \mathrm{MeOH}$

$\mathrm{C}_{63.17} \mathrm{H}_{46.90} \mathrm{Cl}_{2.71} \mathrm{~N}_{6} \mathrm{O}_{2.27}$

1022.27

200(2) K

$0.71073 \AA$

triclinic

$\mathrm{P} \overline{1}$

4

$a=15.5837(8) \AA \quad \alpha=108.0904(13) \mathrm{deg}$.

$b=19.5163(10) \AA \quad \beta=104.3601(13) \mathrm{deg}$.

$c=20.0366(10) \AA \quad \gamma=107.9134(13) \mathrm{deg}$.

5098.8(5) $\AA^{3}$

$1.33 \mathrm{~g} / \mathrm{cm}^{3}$

$0.22 \mathrm{~mm}^{-1}$

plank

$0.061 \times 0.060 \times 0.029 \mathrm{~mm}^{3}$

orange

1.2 to 23.3 deg.

$-17 \leq h \leq 17,-21 \leq k \leq 21,-22 \leq \mathrm{l} \leq 22$

70657

$14656(\mathrm{R}(\mathrm{int})=0.0848)$

$6417(\mathrm{I}>2 \sigma(\mathrm{I}))$

Semi-empirical from equivalents

0.96 and 0.92 
Refinement method

Data/restraints/parameters

Goodness-of-fit on $\mathrm{F}^{2}$

Final $\mathrm{R}$ indices (I>2sigma(I))

Largest diff. peak and hole
Full-matrix least-squares on $\mathrm{F}^{2}$

14656 / 3668 / 1652

1.03

$\mathrm{R} 1=0.084, \mathrm{wR} 2=0.225$

0.81 and $-0.52 \mathrm{e}^{-3}$

Table S12. Atomic coordinates and equivalent isotropic displacement parameters $\left(\AA^{2}\right)$ for QPP-

OMe/PQDC. $U_{\text {eq }}$ is defined as one third of the trace of the orthogonalized $U_{i j}$ tensor.

\begin{tabular}{|c|c|c|c|c|}
\hline Atom & $x$ & $y$ & z & $U_{\text {eq }}$ \\
\hline C111 & $0.6165(4)$ & $0.6609(4)$ & $-0.1014(3)$ & $0.0467(16)$ \\
\hline C121 & $0.6059(4)$ & $0.6402(3)$ & $-0.0402(3)$ & $0.0378(14)$ \\
\hline N131 & $0.5182(3)$ & $0.6230(3)$ & $-0.0327(2)$ & $0.0401(12)$ \\
\hline C141 & $0.5115(4)$ & $0.6036(3)$ & $0.0248(3)$ & $0.0346(14)$ \\
\hline C151 & $0.4179(3)$ & $0.5827(3)$ & $0.0342(3)$ & $0.0301(13)$ \\
\hline C161 & $0.3382(4)$ & $0.5826(3)$ & $-0.0160(3)$ & $0.0396(15)$ \\
\hline H161 & 0.3445 & 0.5950 & -0.0573 & 0.048 \\
\hline C171 & $0.2489(4)$ & $0.5646(3)$ & $-0.0070(3)$ & $0.0375(14)$ \\
\hline C181 & $0.2406(4)$ & $0.5445(3)$ & $0.0526(3)$ & $0.0356(14)$ \\
\hline H181 & 0.1802 & 0.5314 & 0.0591 & 0.043 \\
\hline C191 & $0.3189(3)$ & $0.5432(3)$ & $0.1027(3)$ & $0.0280(13)$ \\
\hline C201 & $0.3090(3)$ & $0.5235(3)$ & $0.1661(3)$ & $0.0251(12)$ \\
\hline N211 & $0.2235(3)$ & $0.5068(2)$ & $0.1740(2)$ & $0.0280(11)$ \\
\hline C221 & $0.2172(3)$ & $0.4901(3)$ & $0.2337(3)$ & $0.0264(12)$ \\
\hline C231 & $0.1295(4)$ & $0.4755(3)$ & $0.2468(3)$ & $0.0279(13)$ \\
\hline H231 & 0.0755 & 0.4773 & 0.2137 & 0.033 \\
\hline C241 & $0.1224(4)$ & $0.4590(3)$ & $0.3070(3)$ & $0.0288(13)$ \\
\hline C251 & $0.0348(4)$ & $0.4434(3)$ & $0.3302(3)$ & $0.0329(13)$ \\
\hline H251 & -0.0219 & 0.4453 & 0.2953 & 0.039 \\
\hline C261 & $0.0110(4)$ & $0.3637(3)$ & $0.3343(3)$ & $0.0307(13)$ \\
\hline C271 & $-0.0776(4)$ & $0.2976(3)$ & $0.2960(3)$ & $0.0364(14)$ \\
\hline $\mathrm{H} 271$ & -0.1307 & 0.2987 & 0.2613 & 0.044 \\
\hline C281 & $-0.0883(4)$ & $0.2306(3)$ & $0.3084(3)$ & $0.0425(15)$ \\
\hline H281 & -0.1493 & 0.1856 & 0.2824 & 0.051 \\
\hline C291 & $-0.0113(4)$ & $0.2281(3)$ & $0.3583(3)$ & $0.0436(15)$ \\
\hline H291 & -0.0196 & 0.1815 & 0.3665 & 0.052 \\
\hline C301 & $0.0775(4)$ & $0.2933(3)$ & $0.3961(3)$ & $0.0374(14)$ \\
\hline H301 & 0.1307 & 0.2915 & 0.4300 & 0.045 \\
\hline C311 & $0.0889(4)$ & $0.3615(3)$ & $0.3843(3)$ & $0.0309(13)$ \\
\hline C321 & $0.0723(4)$ & $0.5048(3)$ & $0.4116(3)$ & $0.0341(13)$ \\
\hline C331 & $0.0351(4)$ & $0.5594(3)$ & $0.4391(3)$ & $0.0421(15)$ \\
\hline H331 & -0.0192 & 0.5607 & 0.4060 & 0.051 \\
\hline C341 & $0.0783(5)$ & $0.6117(3)$ & $0.5153(3)$ & $0.0494(16)$ \\
\hline H341 & 0.0538 & 0.6493 & 0.5346 & 0.059 \\
\hline C351 & $0.1571(4)$ & $0.6091(3)$ & $0.5629(3)$ & $0.0475(16)$ \\
\hline H351 & 0.1861 & 0.6449 & 0.6151 & 0.057 \\
\hline C361 & $0.1943(4)$ & $0.5556(3)$ & $0.5360(3)$ & $0.0385(14)$ \\
\hline H361 & 0.2491 & 0.5549 & 0.5692 & 0.046 \\
\hline C371 & $0.1510(4)$ & $0.5027(3)$ & $0.4601(3)$ & $0.0326(13)$ \\
\hline C381 & $0.1813(4)$ & $0.4388(3)$ & $0.4218(3)$ & $0.0311(13)$ \\
\hline H381 & 0.2373 & 0.4368 & 0.4574 & 0.037 \\
\hline C391 & $0.2024(4)$ & $0.4561(3)$ & $0.3571(3)$ & $0.0289(13)$ \\
\hline C401 & $0.2877(4)$ & $0.4690(3)$ & $0.3462(3)$ & $0.0281(13)$ \\
\hline $\mathrm{H} 401$ & 0.3401 & 0.4658 & 0.3793 & 0.034 \\
\hline C411 & $0.2971(4)$ & $0.4875(3)$ & $0.2840(3)$ & $0.0270(12)$ \\
\hline N421 & $0.3838(3)$ & $0.5046(2)$ & $0.2755(2)$ & $0.0306(11)$ \\
\hline C431 & $0.3899(3)$ & $0.5232(3)$ & $0.2177(3)$ & $0.0273(12)$ \\
\hline C441 & $0.4833(4)$ & $0.5433(3)$ & $0.2084(3)$ & $0.0290(13)$ \\
\hline
\end{tabular}




\begin{tabular}{|c|c|c|c|c|}
\hline C451 & $0.5640(4)$ & $0.5462(3)$ & $0.2597(3)$ & $0.0396(15)$ \\
\hline $\mathrm{H} 451$ & 0.5571 & 0.5331 & 0.3005 & 0.047 \\
\hline C461 & $0.6553(4)$ & $0.5676(3)$ & $0.2538(3)$ & $0.0426(15)$ \\
\hline C471 & $0.6624(4)$ & $0.5851(3)$ & $0.1925(3)$ & $0.0422(15)$ \\
\hline H471 & 0.7230 & 0.5986 & 0.1863 & 0.051 \\
\hline C481 & $0.5828(4)$ & $0.5832(3)$ & $0.1401(3)$ & $0.0321(13)$ \\
\hline C491 & $0.5926(4)$ & $0.6038(3)$ & $0.0770(3)$ & $0.0326(13)$ \\
\hline N501 & $0.6788(3)$ & $0.6229(3)$ & $0.0700(3)$ & $0.0416(12)$ \\
\hline C511 & $0.6865(4)$ & $0.6409(3)$ & $0.0112(3)$ & $0.0385(14)$ \\
\hline C521 & $0.7774(4)$ & $0.6601(3)$ & $0.0009(3)$ & $0.0426(15)$ \\
\hline C531 & $0.7835(4)$ & $0.6780(3)$ & $-0.0584(3)$ & $0.0462(16)$ \\
\hline H531 & 0.8434 & 0.6906 & -0.0656 & 0.055 \\
\hline C541 & $0.7036(4)$ & $0.6785(3)$ & $-0.1097(3)$ & $0.0480(16)$ \\
\hline H541 & 0.7108 & 0.6913 & -0.1506 & 0.058 \\
\hline C551 & $0.4094(3)$ & $0.5628(3)$ & $0.0947(3)$ & $0.0268(12)$ \\
\hline C561 & $0.4921(3)$ & $0.5631(3)$ & $0.1475(3)$ & $0.0277(12)$ \\
\hline C571 & $0.1609(4)$ & $0.5639(4)$ & $-0.0626(3)$ & $0.065(2)$ \\
\hline C5711 & $0.1036(10)$ & $0.4822(6)$ & $-0.1238(7)$ & $0.338(12)$ \\
\hline H57A1 & 0.1454 & 0.4662 & -0.1488 & 0.507 \\
\hline H57B1 & 0.0794 & 0.4455 & -0.1018 & 0.507 \\
\hline H57C1 & 0.0483 & 0.4811 & -0.1611 & 0.507 \\
\hline C5721 & $0.1851(6)$ & $0.6187(5)$ & $-0.0983(5)$ & $0.113(3)$ \\
\hline H57D1 & 0.2265 & 0.6062 & -0.1254 & 0.169 \\
\hline H57E1 & 0.1250 & 0.6131 & -0.1342 & 0.169 \\
\hline H57F1 & 0.2199 & 0.6733 & -0.0592 & 0.169 \\
\hline C5731 & $0.1004(9)$ & $0.5886(12)$ & $-0.0251(7)$ & $0.352(12)$ \\
\hline H57G1 & 0.0805 & 0.5548 & 0.0006 & 0.528 \\
\hline $\mathrm{H} 57 \mathrm{H} 1$ & 0.1373 & 0.6439 & 0.0122 & 0.528 \\
\hline H57I1 & 0.0424 & 0.5837 & -0.0628 & 0.528 \\
\hline C581 & $0.7423(4)$ & $0.5690(4)$ & $0.3116(4)$ & $0.0560(18)$ \\
\hline C5811 & $0.8386(5)$ & $0.6230(6)$ & $0.3141(6)$ & $0.143(5)$ \\
\hline H58A1 & 0.8400 & 0.6060 & 0.2631 & 0.215 \\
\hline H58B1 & 0.8451 & 0.6778 & 0.3322 & 0.215 \\
\hline H58C1 & 0.8927 & 0.6199 & 0.3486 & 0.215 \\
\hline C5821 & $0.7398(5)$ & $0.5952(4)$ & $0.3906(4)$ & $0.082(2)$ \\
\hline H58D1 & 0.6778 & 0.5604 & 0.3889 & 0.123 \\
\hline H58E1 & 0.7938 & 0.5921 & 0.4252 & 0.123 \\
\hline H58F1 & 0.7462 & 0.6500 & 0.4088 & 0.123 \\
\hline C5831 & $0.7329(5)$ & $0.4838(4)$ & $0.2856(4)$ & $0.068(2)$ \\
\hline H58G1 & 0.7343 & 0.4655 & 0.2347 & 0.102 \\
\hline $\mathrm{H} 58 \mathrm{H} 1$ & 0.7872 & 0.4818 & 0.3210 & 0.102 \\
\hline H58I1 & 0.6711 & 0.4495 & 0.2846 & 0.102 \\
\hline O591 & $0.5344(3)$ & $0.6596(3)$ & $-0.1475(2)$ & $0.0672(14)$ \\
\hline C591 & $0.5407(5)$ & $0.6762(5)$ & $-0.2105(4)$ & $0.091(3)$ \\
\hline H59A1 & 0.4767 & 0.6694 & -0.2417 & 0.137 \\
\hline H59B1 & 0.5618 & 0.6398 & -0.2410 & 0.137 \\
\hline H59C1 & 0.5881 & 0.7310 & -0.1925 & 0.137 \\
\hline O601 & $0.8509(3)$ & $0.6592(3)$ & $0.0530(3)$ & $0.0564(12)$ \\
\hline C601 & $0.9410(5)$ & $0.6769(5)$ & $0.0420(5)$ & $0.082(3)$ \\
\hline H60A1 & 0.9873 & 0.6700 & 0.0796 & 0.123 \\
\hline H60B1 & 0.9678 & 0.7319 & 0.0479 & 0.123 \\
\hline H60C1 & 0.9297 & 0.6411 & -0.0092 & 0.123 \\
\hline C611 & $0.6314(7)$ & $0.6789(6)$ & $0.5488(7)$ & $0.065(3)$ \\
\hline N611 & $0.6949(7)$ & $0.6801(6)$ & $0.5926(7)$ & $0.098(4)$ \\
\hline C621 & $0.5458(8)$ & $0.6788(9)$ & $0.4988(7)$ & $0.051(3)$ \\
\hline N631 & $0.5579(9)$ & $0.7018(8)$ & $0.4445(7)$ & $0.048(3)$ \\
\hline C641 & $0.4784(6)$ & $0.7021(4)$ & $0.3986(5)$ & $0.0363(18)$ \\
\hline C651 & $0.4834(6)$ & $0.7236(5)$ & $0.3353(5)$ & $0.040(2)$ \\
\hline C661 & $0.5697(6)$ & $0.7418(5)$ & $0.3211(5)$ & $0.041(2)$ \\
\hline H661 & 0.6268 & 0.7423 & 0.3527 & 0.049 \\
\hline C671 & $0.5676(8)$ & $0.7592(12)$ & $0.2583(8)$ & $0.055(3)$ \\
\hline
\end{tabular}




\begin{tabular}{|c|c|c|c|c|}
\hline H671 & 0.6249 & 0.7708 & 0.2472 & 0.067 \\
\hline C681 & $0.4910(7)$ & $0.7604(6)$ & $0.2135(5)$ & $0.053(2)$ \\
\hline H681 & 0.4952 & 0.7745 & 0.1727 & 0.063 \\
\hline C691 & $0.4012(8)$ & $0.7407(11)$ & $0.2256(8)$ & $0.050(3)$ \\
\hline C701 & $0.3177(7)$ & $0.7393(5)$ & $0.1770(5)$ & $0.056(2)$ \\
\hline H701 & 0.3201 & 0.7511 & 0.1347 & 0.068 \\
\hline C711 & $0.2328(7)$ & $0.7210(5)$ & $0.1900(5)$ & $0.055(2)$ \\
\hline H711 & 0.1762 & 0.7193 & 0.1565 & 0.066 \\
\hline C721 & $0.2303(7)$ & $0.7047(6)$ & $0.2542(6)$ & $0.047(2)$ \\
\hline C731 & $0.1436(8)$ & $0.6866(6)$ & $0.2696(7)$ & $0.056(3)$ \\
\hline H731 & 0.0867 & 0.6851 & 0.2368 & 0.068 \\
\hline C741 & $0.1421(9)$ & $0.6719(11)$ & $0.3288(9)$ & $0.056(3)$ \\
\hline $\mathrm{H} 741$ & 0.0838 & 0.6621 & 0.3385 & 0.067 \\
\hline C751 & $0.2202(6)$ & $0.6699(4)$ & $0.3782(5)$ & $0.040(2)$ \\
\hline H751 & 0.2163 & 0.6587 & 0.4205 & 0.048 \\
\hline C761 & $0.3061(11)$ & $0.6853(14)$ & $0.3625(10)$ & $0.040(3)$ \\
\hline C771 & $0.3908(8)$ & $0.6831(8)$ & $0.4109(7)$ & $0.038(2)$ \\
\hline N781 & $0.3811(5)$ & $0.6585(4)$ & $0.4662(4)$ & $0.0385(18)$ \\
\hline C791 & $0.4602(7)$ & $0.6562(6)$ & $0.5081(6)$ & $0.044(2)$ \\
\hline C801 & $0.4519(7)$ & $0.6249(8)$ & $0.5652(7)$ & $0.057(3)$ \\
\hline N801 & $0.4440(6)$ & $0.5981(6)$ & $0.6061(5)$ & $0.090(3)$ \\
\hline C811 & $0.3997(6)$ & $0.7218(4)$ & $0.2882(4)$ & $0.0363(18)$ \\
\hline C821 & $0.3109(6)$ & $0.7033(4)$ & $0.3022(4)$ & $0.0374(19)$ \\
\hline C911 & $0.8744(7)$ & $0.7854(7)$ & $0.2148(6)$ & $0.053(3)$ \\
\hline H911 & 0.8246 & 0.7308 & 0.1782 & 0.063 \\
\hline $\mathrm{Cl} 11$ & $0.8963(2)$ & $0.8441(3)$ & $0.1649(2)$ & $0.0636(9)$ \\
\hline $\mathrm{Cl} 21$ & $0.9822(2)$ & $0.7796(2)$ & $0.2598(2)$ & $0.0711(9)$ \\
\hline Cl31 & $0.8314(2)$ & $0.8240(2)$ & $0.2833(2)$ & $0.0637(9)$ \\
\hline C921 & $0.4656(8)$ & $0.7803(10)$ & $-0.0489(7)$ & $0.127(7)$ \\
\hline H921 & 0.4632 & 0.7263 & -0.0583 & 0.152 \\
\hline Cl41 & $0.5803(5)$ & $0.8426(3)$ & $-0.0357(5)$ & $0.173(4)$ \\
\hline Cl51 & $0.3789(5)$ & $0.7744(4)$ & $-0.1301(4)$ & $0.181(3)$ \\
\hline Cl61 & $0.4401(5)$ & $0.8150(6)$ & $0.0314(4)$ & $0.130(3)$ \\
\hline C931 & $0.629(2)$ & $0.820(3)$ & $0.075(2)$ & $0.105(10)$ \\
\hline 0941 & $0.707(2)$ & $0.816(2)$ & $0.1391(19)$ & $0.178(11)$ \\
\hline C93B1 & $0.372(4)$ & $0.678(5)$ & $0.420(4)$ & $0.072(16)$ \\
\hline O94B1 & $0.289(3)$ & $0.686(4)$ & $0.364(3)$ & $0.031(10)$ \\
\hline C61B1 & $0.3679(19)$ & $0.826(2)$ & $-0.0491(17)$ & $0.127(8)$ \\
\hline N61B1 & $0.310(2)$ & $0.8180(18)$ & $-0.1023(17)$ & $0.157(11)$ \\
\hline C62B1 & $0.4514(18)$ & $0.821(3)$ & $0.000(2)$ & $0.115(8)$ \\
\hline N63B1 & $0.443(2)$ & $0.803(3)$ & $0.057(2)$ & $0.114(8)$ \\
\hline C64B1 & $0.5230(15)$ & $0.7971(15)$ & $0.0970(12)$ & $0.104(6)$ \\
\hline C65B1 & $0.5180(14)$ & $0.7764(14)$ & $0.1603(11)$ & $0.093(6)$ \\
\hline C66B1 & $0.4302(15)$ & $0.7567(15)$ & $0.1720(13)$ & $0.098(6)$ \\
\hline H66B1 & 0.3726 & 0.7538 & 0.1386 & 0.117 \\
\hline C67B1 & $0.433(2)$ & $0.742(3)$ & $0.236(2)$ & $0.087(7)$ \\
\hline H67B1 & 0.3755 & 0.7300 & 0.2464 & 0.105 \\
\hline C68B1 & $0.5109(15)$ & $0.7430(14)$ & $0.2824(13)$ & $0.083(6)$ \\
\hline H68B1 & 0.5069 & 0.7309 & 0.3242 & 0.100 \\
\hline C69B1 & $0.6002(18)$ & $0.762(3)$ & $0.2712(18)$ & $0.081(6)$ \\
\hline C70B1 & $0.6839(15)$ & $0.7631(15)$ & $0.3198(12)$ & $0.105(7)$ \\
\hline H70B1 & 0.6819 & 0.7535 & 0.3632 & 0.126 \\
\hline C71B1 & $0.7697(16)$ & $0.7778(16)$ & $0.3057(13)$ & $0.109(7)$ \\
\hline H71B1 & 0.8238 & 0.7739 & 0.3362 & 0.131 \\
\hline C72B1 & $0.7738(16)$ & $0.7987(17)$ & $0.2445(13)$ & $0.098(6)$ \\
\hline C73B1 & $0.8571(18)$ & $0.8130(18)$ & $0.2255(16)$ & $0.092(8)$ \\
\hline H73B1 & 0.9128 & 0.8105 & 0.2555 & 0.111 \\
\hline C74B1 & $0.859(2)$ & $0.830(3)$ & $0.1673(19)$ & $0.100(8)$ \\
\hline H74B1 & 0.9182 & 0.8427 & 0.1591 & 0.120 \\
\hline C75B1 & $0.7800(16)$ & $0.8301(14)$ & $0.1167(14)$ & $0.096(6)$ \\
\hline Н75B1 & 0.7831 & 0.8408 & 0.0741 & 0.116 \\
\hline
\end{tabular}




\begin{tabular}{|c|c|c|c|c|}
\hline C76B1 & $0.6949(16)$ & $0.8133(18)$ & $0.1333(14)$ & $0.088(5)$ \\
\hline C77B1 & $0.6103(15)$ & $0.8187(17)$ & $0.0881(13)$ & $0.085(5)$ \\
\hline N78B1 & $0.6167(14)$ & $0.8374(12)$ & $0.0296(11)$ & $0.090(6)$ \\
\hline C79B1 & $0.5357(17)$ & $0.837(2)$ & $-0.0127(16)$ & $0.111(7)$ \\
\hline C80B1 & $0.542(3)$ & $0.875(2)$ & $-0.0660(19)$ & $0.121(10)$ \\
\hline N80B1 & $0.546(2)$ & $0.9112(18)$ & $-0.1002(16)$ & $0.209(17)$ \\
\hline C81B1 & $0.6010(13)$ & $0.7779(14)$ & $0.2072(11)$ & $0.096(6)$ \\
\hline C82B1 & $0.6906(13)$ & $0.7983(15)$ & $0.1958(12)$ & $0.098(6)$ \\
\hline C91B1 & $0.1291(14)$ & $0.7173(14)$ & $0.2902(11)$ & $0.090(9)$ \\
\hline H91B1 & 0.1796 & 0.7715 & 0.3272 & 0.109 \\
\hline Cl1B1 & $0.1051(9)$ & $0.6578(9)$ & $0.3380(7)$ & $0.114(4)$ \\
\hline Cl2B1 & $0.0219(7)$ & $0.7238(7)$ & $0.2460(7)$ & $0.167(4)$ \\
\hline Cl3B1 & $0.1691(7)$ & $0.6785(6)$ & $0.2207(5)$ & $0.110(3)$ \\
\hline C92B1 & $0.5396(12)$ & $0.7192(16)$ & $0.5499(11)$ & $0.096(10)$ \\
\hline H92B1 & 0.5422 & 0.7733 & 0.5590 & 0.115 \\
\hline Cl4B1 & $0.4228(11)$ & $0.6563(10)$ & $0.5333(10)$ & $0.160(7)$ \\
\hline Cl5B1 & $0.6257(10)$ & $0.7257(10)$ & $0.6289(8)$ & $0.179(7)$ \\
\hline Cl6B1 & $0.5639(14)$ & $0.6835(14)$ & $0.4690(10)$ & $0.137(8)$ \\
\hline C112 & $0.3934(4)$ & $0.8315(4)$ & $0.6050(3)$ & $0.0473(16)$ \\
\hline C122 & $0.4040(4)$ & $0.8545(3)$ & $0.5452(3)$ & $0.0361(14)$ \\
\hline N132 & $0.4892(3)$ & $0.8713(3)$ & $0.5351(2)$ & $0.0346(11)$ \\
\hline C142 & $0.4949(4)$ & $0.8943(3)$ & $0.4795(3)$ & $0.0308(13)$ \\
\hline C152 & $0.5875(4)$ & $0.9138(3)$ & $0.4680(3)$ & $0.0302(13)$ \\
\hline C162 & $0.6673(4)$ & $0.9095(3)$ & $0.5138(3)$ & $0.0367(14)$ \\
\hline H162 & 0.6620 & 0.8953 & 0.5544 & 0.044 \\
\hline C172 & $0.7545(4)$ & $0.9252(3)$ & $0.5023(3)$ & $0.0387(14)$ \\
\hline C182 & $0.7603(4)$ & $0.9479(3)$ & $0.4437(3)$ & $0.0350(14)$ \\
\hline H182 & 0.8198 & 0.9606 & 0.4357 & 0.042 \\
\hline C192 & $0.6829(4)$ & $0.9530(3)$ & $0.3964(3)$ & $0.0283(13)$ \\
\hline C202 & $0.6903(4)$ & $0.9744(3)$ & $0.3335(3)$ & $0.0275(13)$ \\
\hline N212 & $0.7746(3)$ & $0.9902(2)$ & $0.3234(2)$ & $0.0287(11)$ \\
\hline C222 & $0.7794(4)$ & $1.0074(3)$ & $0.2633(3)$ & $0.0269(12)$ \\
\hline C232 & $0.8658(4)$ & $1.0221(3)$ & $0.2494(3)$ & $0.0285(13)$ \\
\hline $\mathrm{H} 232$ & 0.9205 & 1.0206 & 0.2819 & 0.034 \\
\hline C242 & $0.8701(4)$ & $1.0385(3)$ & $0.1887(3)$ & $0.0298(13)$ \\
\hline C252 & $0.9577(4)$ & $1.0554(3)$ & $0.1650(3)$ & $0.0352(13)$ \\
\hline $\mathrm{H} 252$ & 1.0150 & 1.0543 & 0.1999 & 0.042 \\
\hline C262 & $0.9210(4)$ & $0.9941(3)$ & $0.0840(3)$ & $0.0326(13)$ \\
\hline C272 & $0.9590(4)$ & $0.9406(3)$ & $0.0565(3)$ & $0.0398(14)$ \\
\hline $\mathrm{H} 272$ & 1.0139 & 0.9400 & 0.0898 & 0.048 \\
\hline C282 & $0.9173(5)$ & $0.8884(3)$ & $-0.0191(3)$ & $0.0480(16)$ \\
\hline $\mathrm{H} 282$ & 0.9436 & 0.8520 & -0.0378 & 0.058 \\
\hline C292 & $0.8375(5)$ & $0.8889(3)$ & $-0.0675(3)$ & $0.0484(16)$ \\
\hline H292 & 0.8089 & 0.8526 & -0.1195 & 0.058 \\
\hline C302 & $0.7986(4)$ & $0.9417(3)$ & $-0.0409(3)$ & $0.0418(15)$ \\
\hline H302 & 0.7435 & 0.9417 & -0.0744 & 0.050 \\
\hline C312 & $0.8406(4)$ & $0.9947(3)$ & $0.0351(3)$ & $0.0349(13)$ \\
\hline C322 & $0.9802(4)$ & $1.1346(3)$ & $0.1604(3)$ & $0.0307(13)$ \\
\hline C332 & $1.0678(4)$ & $1.2017(3)$ & $0.1990(3)$ & $0.0374(14)$ \\
\hline H332 & 1.1211 & 1.2019 & 0.2346 & 0.045 \\
\hline C342 & $1.0773(4)$ & $1.2680(3)$ & $0.1856(3)$ & $0.0445(15)$ \\
\hline H342 & 1.1377 & 1.3136 & 0.2111 & 0.053 \\
\hline C352 & $0.9991(5)$ & $1.2685(3)$ & $0.1350(3)$ & $0.0472(16)$ \\
\hline H352 & 1.0063 & 1.3145 & 0.1258 & 0.057 \\
\hline C362 & $0.9106(4)$ & $1.2030(3)$ & $0.0977(3)$ & $0.0404(14)$ \\
\hline H362 & 0.8569 & 1.2039 & 0.0635 & 0.049 \\
\hline C372 & $0.9011(4)$ & $1.1365(3)$ & $0.1108(3)$ & $0.0320(13)$ \\
\hline C382 & $0.8099(4)$ & $1.0580(3)$ & $0.0741(3)$ & $0.0348(13)$ \\
\hline H382 & 0.7531 & 1.0592 & 0.0386 & 0.042 \\
\hline C392 & $0.7896(4)$ & $1.0414(3)$ & $0.1395(3)$ & $0.0322(13)$ \\
\hline C402 & $0.7052(4)$ & $1.0282(3)$ & $0.1520(3)$ & $0.0341(14)$ \\
\hline
\end{tabular}




\begin{tabular}{|c|c|c|c|c|}
\hline $\mathrm{H} 402$ & 0.6516 & 1.0305 & 0.1191 & 0.041 \\
\hline C412 & $0.6980(4)$ & $1.0110(3)$ & $0.2150(3)$ & $0.0299(13)$ \\
\hline N422 & $0.6135(3)$ & $0.9958(2)$ & $0.2261(2)$ & $0.0322(11)$ \\
\hline C432 & $0.6092(4)$ & $0.9774(3)$ & $0.2846(3)$ & $0.0293(13)$ \\
\hline C442 & $0.5178(4)$ & $0.9594(3)$ & $0.2977(3)$ & $0.0319(13)$ \\
\hline C452 & $0.4377(4)$ & $0.9609(3)$ & $0.2502(3)$ & $0.0389(15)$ \\
\hline $\mathrm{H} 452$ & 0.4432 & 0.9754 & 0.2098 & 0.047 \\
\hline C462 & $0.3492(4)$ & $0.9416(4)$ & $0.2601(3)$ & $0.0444(15)$ \\
\hline C472 & $0.3445(4)$ & $0.9224(3)$ & $0.3205(3)$ & $0.0419(15)$ \\
\hline H472 & 0.2850 & 0.9096 & 0.3283 & 0.050 \\
\hline C482 & $0.4233(4)$ & $0.9212(3)$ & $0.3700(3)$ & $0.0330(13)$ \\
\hline C492 & $0.4157(4)$ & $0.8990(3)$ & $0.4323(3)$ & $0.0312(13)$ \\
\hline N502 & $0.3303(3)$ & $0.8818(2)$ & $0.4410(2)$ & $0.0352(11)$ \\
\hline C512 & $0.3246(4)$ & $0.8605(3)$ & $0.4986(3)$ & $0.0361(14)$ \\
\hline C522 & $0.2352(4)$ & $0.8430(3)$ & $0.5116(3)$ & $0.0415(15)$ \\
\hline C532 & $0.2299(5)$ & $0.8223(3)$ & $0.5692(3)$ & $0.0473(16)$ \\
\hline H532 & 0.1715 & 0.8117 & 0.5787 & 0.057 \\
\hline C542 & $0.3082(4)$ & $0.8161(3)$ & $0.6154(3)$ & $0.0494(16)$ \\
\hline H542 & 0.3013 & 0.8007 & 0.6550 & 0.059 \\
\hline C552 & $0.5942(4)$ & $0.9359(3)$ & $0.4082(3)$ & $0.0282(12)$ \\
\hline C562 & $0.5123(4)$ & $0.9391(3)$ & $0.3587(3)$ & $0.0288(13)$ \\
\hline C572 & $0.8408(4)$ & $0.9170(4)$ & $0.5498(3)$ & $0.0597(19)$ \\
\hline C5712 & $0.8232(5)$ & $0.8900(5)$ & $0.6089(4)$ & $0.074(2)$ \\
\hline H57A2 & 0.8806 & 0.8843 & 0.6357 & 0.111 \\
\hline H57B2 & 0.7664 & 0.8389 & 0.5849 & 0.111 \\
\hline $\mathrm{H} 57 \mathrm{C} 2$ & 0.8112 & 0.9293 & 0.6452 & 0.111 \\
\hline C5722 & $0.9307(5)$ & $0.9918(5)$ & $0.5813(5)$ & $0.144(5)$ \\
\hline H57D2 & 0.9850 & 0.9873 & 0.6140 & 0.216 \\
\hline H57E2 & 0.9197 & 1.0364 & 0.6111 & 0.216 \\
\hline H57F2 & 0.9464 & 1.0011 & 0.5395 & 0.216 \\
\hline C5732 & $0.8575(7)$ & $0.8493(6)$ & $0.4957(5)$ & $0.122(4)$ \\
\hline H57G2 & 0.9139 & 0.8439 & 0.5244 & 0.183 \\
\hline $\mathrm{H} 57 \mathrm{H} 2$ & 0.8695 & 0.8625 & 0.4546 & 0.183 \\
\hline H57I2 & 0.7994 & 0.7993 & 0.4743 & 0.183 \\
\hline C582 & $0.2627(4)$ & $0.9452(4)$ & $0.2066(4)$ & $0.0576(18)$ \\
\hline C5812 & $0.1679(5)$ & $0.9047(6)$ & $0.2156(5)$ & $0.115(4)$ \\
\hline H58A2 & 0.1158 & 0.9138 & 0.1859 & 0.172 \\
\hline H58B2 & 0.1771 & 0.9269 & 0.2693 & 0.172 \\
\hline H58C2 & 0.1502 & 0.8476 & 0.1974 & 0.172 \\
\hline C5822 & $0.2800(5)$ & $1.0307(4)$ & $0.2243(4)$ & $0.076(2)$ \\
\hline H58D2 & 0.2232 & 1.0324 & 0.1915 & 0.113 \\
\hline H58E2 & 0.3379 & 1.0569 & 0.2154 & 0.113 \\
\hline H58F2 & 0.2901 & 1.0583 & 0.2776 & 0.113 \\
\hline C5832 & $0.2493(5)$ & $0.9046(5)$ & $0.1246(4)$ & $0.089(3)$ \\
\hline H58G2 & 0.1990 & 0.9131 & 0.0920 & 0.134 \\
\hline $\mathrm{H} 58 \mathrm{H} 2$ & 0.2293 & 0.8476 & 0.1100 & 0.134 \\
\hline H58I2 & 0.3109 & 0.9268 & 0.1185 & 0.134 \\
\hline O592 & $0.4722(3)$ & $0.8257(3)$ & $0.6463(2)$ & $0.0673(14)$ \\
\hline C592 & $0.4647(5)$ & $0.8071(5)$ & $0.7086(4)$ & $0.096(3)$ \\
\hline H59A2 & 0.5231 & 0.8008 & 0.7325 & 0.143 \\
\hline H59B2 & 0.4070 & 0.7575 & 0.6903 & 0.143 \\
\hline H59C2 & 0.4589 & 0.8500 & 0.7458 & 0.143 \\
\hline $\mathrm{O} 602$ & $0.1625(3)$ & $0.8485(2)$ & $0.4613(2)$ & $0.0551(12)$ \\
\hline C602 & $0.0739(4)$ & $0.8337(4)$ & $0.4738(4)$ & $0.073(2)$ \\
\hline $\mathrm{H} 60 \mathrm{~A} 2$ & 0.0306 & 0.8465 & 0.4400 & 0.109 \\
\hline H60B2 & 0.0880 & 0.8667 & 0.5267 & 0.109 \\
\hline $\mathrm{H} 60 \mathrm{C} 2$ & 0.0422 & 0.7776 & 0.4634 & 0.109 \\
\hline
\end{tabular}




\section{Theoretical Calculations}

\subsection{Degree of Charge Transfer}

The degree of charge transfer (Ionicity) between the donor and acceptor molecules, which is defined as the sum of all atomic charges on the donor part of the complex, ${ }^{[S 9]}$ is obtained using Mulliken population analysis computed by B3LYP functional and 6$31 \mathrm{G}(\mathrm{d}, \mathrm{p})$ basis set, using the Gaussian 09 package.

\subsection{Intermolecular Frontier Orbitals}
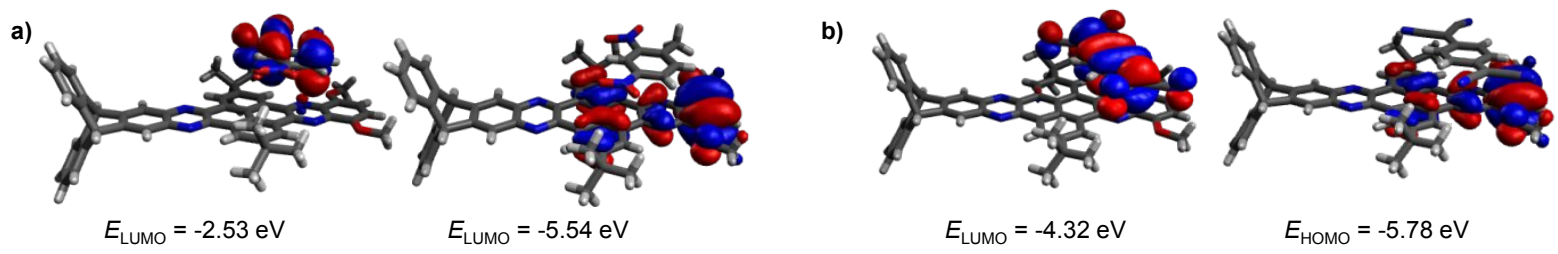

Figure S18. Intermolecular frontier orbitals (DFT-PBE0/6-31G**) of a) QPP-OMe/DNT (dimer B) and QPP-OMe/TCNQ (dimer B).

\subsection{Electronic Coupling}

\subsubsection{Direct Coupling of $\pi$ Stacked Dimers (DFTB)}

The calculation of the electronic couplings requires quantum chemical methods. In this work, the semi-empirical Tight-Binding Density Functional theory (DFTB) method was applied to calculate transfer integrals between $\pi$ stacked donor-acceptor dimers. [S10] This method is derived from density functional theory (DFT) but roughly 2-3 orders of magnitude faster than standard GGA-DFT methods with medium sized basis sets. Therefore, the individual molecules, which contain $\sim 100$ atoms, can be calculated in reasonable computational time, allowing to compute the extensive scans for the dimers containing up to 200 atoms.

For the calculation of the charge transfer couplings, the complex is separated into fragments, corresponding to the individual molecules. For each of the $M$ fragments (molecules, indexed as $m$ ), we compute the molecular orbitals $\varphi_{m}^{i}$

$$
\varphi_{m}^{i}=\sum_{\mu} c_{\mu}^{i m} \chi_{\mu} .
$$

Here, the $i$-th molecular orbital (FO) of fragment $m$ is expressed in an atomic-orbitallike basis set $\chi_{\mu}$ with expansion coefficients $c_{\mu}^{i m}$. Usually, it is sufficient to consider for each fragment one orbital (FO), which will be the HOMO for a hole transfer and the LUMO for an electron transfer $\left(\varphi_{m}^{L U M O}\right.$ or $\left.\varphi_{m}^{H O M O}\right)$. In special cases, more orbitals (like HOMO-1 etc.) can also be taken into account. This can be especially relevant, if the energy difference between the orbitals is small. The Hamiltonian matrix is built from the FO coefficients, e.g. for the coupling of the HOMO orbitals one gets

$$
H_{m n}=\left\langle\varphi_{m}^{\text {HOMO }}|\hat{H}| \varphi_{n}^{\text {HOMO }}\right\rangle=\sum_{\mu} \sum_{v} c_{\mu}^{\text {НОМО }}{ }^{\text {HOMO }}{ }^{\text {HOMO }} \bar{H}_{\mu v} .
$$


The off-diagonal elements of the Hamiltonian matrix correspond to the electronic couplings between the individual fragments, the diagonal elements are the orbital energies. $\bar{H}_{\mu \nu}$ is the Hamiltonian in the atomic-orbital basis.

Using the fast, semi-empirical DFTB method to compute $\bar{H}_{\mu \nu}$ leads to a highly efficient scheme to compute couplings, allowing to treat large systems and investigate a multitude of conformations. In a recent extended benchmark study it has been shown, that this approximate methodology reproduces the couplings, computed with high level ab initio methods for a large molecular test set, with high accuracy. [S11-12]

Beside the successful benchmark of the coupling calculation, this FO-DFTB approach was also used to perform direct simulations of the charge carrier in different systems e.g. organic semiconductors, DNA and proteins, which reproduced the experimental results. [S13-20]

DFT is known to be an approximate method, which shows excellent performance for many molecular properties like geometries or thermochemistry data, but, on the other hand, is known to exhibit several short comings. The most prominent among these is the self-interaction error of DFT, present in DFT functionals using the generalized gradient approximation (GGA), which leads to a wrong estimate of orbital energies. Compared to ab initio methods, not only the absolute energies are deviant, but often also the relative ordering of the orbitals. This behavior of GGA functionals like BLYP or PBE is - only partially - corrected for when using hybrid functionals like B3LPY.

Typically, the energies of occupied orbitals are too high (by several eV!), and the energy differences between orbitals are too small and/or orbitals are interchanged. The character and shape of the orbitals, however, is preserved. There, in DFT-GGA often the HOMO orbital has a $\sigma$-symmetry, while the correct $\pi$-orbital can be found as HOMO-1 or HOMO-2. This deficiency is particularly common in large molecules like those considered in this work (in fact, the energy differences between the orbitals is as little as $0.02-0.15 \mathrm{eV}$ ). To compute the correct charge transfer couplings, the respective orbitals with the proper symmetry have to be used. This problem also occurs in DFTB, since it is derived from DFT-GGA using a PBE functional. [S21-22]

To this end, each molecule was placed in the $x, y$-plane of the coordinate system, and the contribution of the $\pi_{z}$-orbitals on the heavy atoms of the conjugated system to the LUMO, HOMO, ..., HOMO-3 was calculated from their LCAO coefficients as $\sum_{\mu}\left(c_{\mu \pi_{z}}^{i m}\right)^{2}$. The orbital was considered to have $\pi$-symmetry if that value exceeded a threshold (0.1). The DFTB+ program was employed and to visualize the orbitals and to calculate the coefficients. ${ }^{[\mathrm{S} 20]}$ 
a)

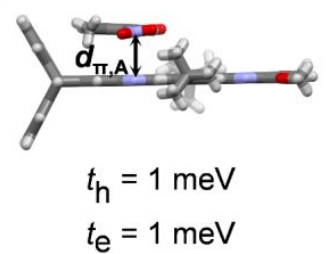

b)

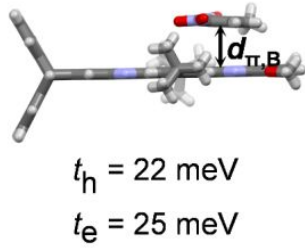

c)

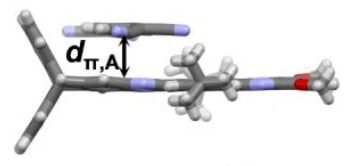

$t_{\mathrm{h}}=1 \mathrm{meV}$

$t_{\mathrm{e}}=1 \mathrm{meV}$

d)

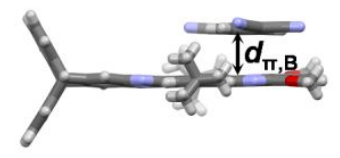

$t_{\mathrm{h}}=0 \mathrm{meV}$

$t_{\mathrm{e}}=0 \mathrm{meV}$ e)

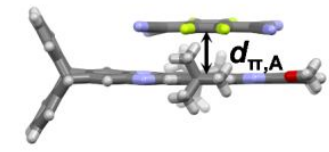

$t_{\mathrm{h}}=3 \mathrm{meV}$

$t_{\mathrm{e}}=3 \mathrm{meV}$

f)

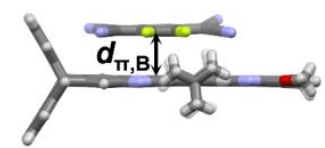

$t_{\mathrm{h}}=16 \mathrm{meV}$

$t_{\mathrm{e}}=19 \mathrm{meV}$ g)

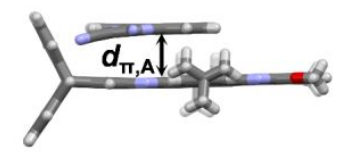

$t_{\mathrm{h}}=5 \mathrm{meV}$

$t_{\mathrm{e}}=6 \mathrm{meV}$

h)

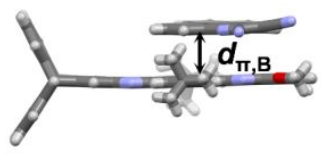

$t_{\mathrm{h}}=50 \mathrm{meV}$

$t_{\mathrm{e}}=58 \mathrm{meV}$

Figure S19. Donor acceptor pairs in the XRD structures of a,b) QPP-OMe/DNT, c,d) QPP-OMe/TCNQ, e,f) QPP-OMe/F ${ }_{4}$ TCNQ and g,h) QPP-OMe/PQDC and corresponding transfer integrals for hole and electron transport.

\subsubsection{Superexchange Coupling (Effective Transfer Integrals)}

In order to calculate the effective electronic coupling for the holes (and electrons) which results from the mixing of the frontier orbitals of two closest donor (acceptor) molecules along the stacking direction with the orbitals of the bridging acceptor (donor) molecule, an energy-splitting approach by considering the energy levels of a complex system, which consists of actual sequence of donor-acceptor molecules in the crystal, is considered. ${ }^{[\mathrm{S} 23]}$

$$
\begin{gathered}
V_{\text {Hole }}=\frac{E_{\text {HОМO }}-E_{\text {HOMO }-1}}{2} \\
V_{\text {Electron }}=\frac{E_{L U M O+1}-E_{L U M O}}{2}
\end{gathered}
$$

Due to the large number of atoms in the unit cell, the experimental geometry of the SCXRD structures was adopted for all the structures. The effective transfer integrals for nearest-neighbor pairs of donor-acceptor molecules were evaluated with the B3LYP functional and 6-31G(d,p) basis set, using the Gaussian 09 package. 


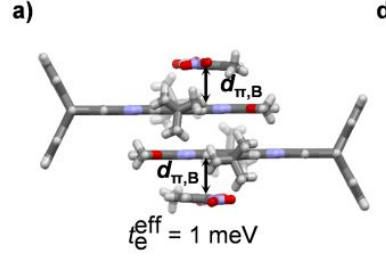

b)

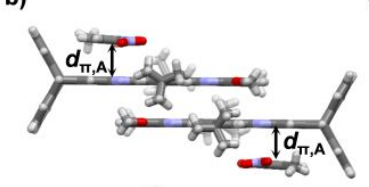

$t_{\mathrm{e}}^{\text {eff }}=0 \mathrm{meV}$

c)

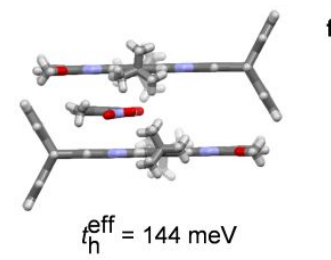

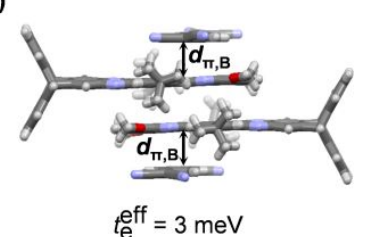

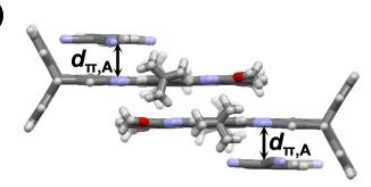

$t_{\mathrm{e}}^{\text {eff }}=0 \mathrm{meV}$

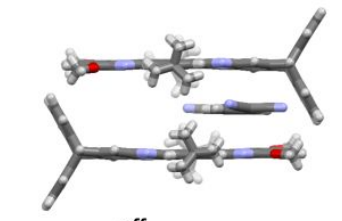

$t_{\mathrm{h}}^{\text {eff }}=99 \mathrm{meV}$ g)

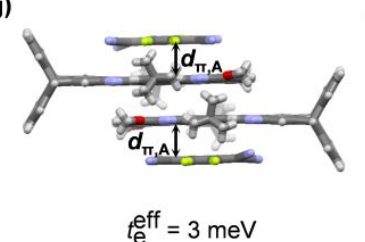

h)

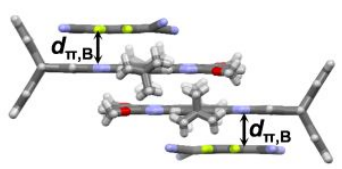

$t_{e}^{e f f}=4 \mathrm{meV}$

i)

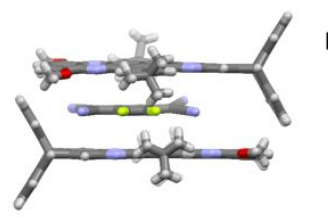

$t_{\mathrm{h}}^{\text {eff }}=67 \mathrm{meV}$

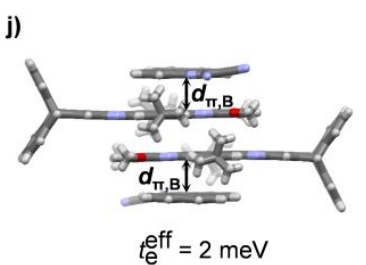

k)

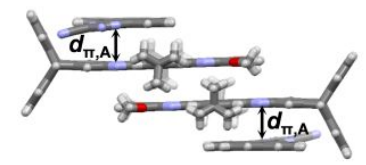

$t_{\mathrm{e}}^{\mathrm{eff}^{\mathrm{f}}}=0 \mathrm{meV}$

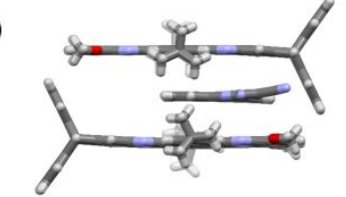

$t_{\mathrm{h}}^{\text {eff }}=18 \mathrm{meV}$

Figure S20. Clusters used for calculation of superexchange couplings (DFT-B3LYP/6-31G**) in the XRD structures of a-c) QPP-OMe/DNT, d-f) QPP-OMe/TCNQ, g-i) QPP-OMe/F4TCNQ and j-I) QPPOMe/PQDC. For electron coupling (e) a A/D/D/A cluster, for hole coupling (h) a D-A-D triad was used.

\section{References}

[S1] CrysAlisPro, Agilent Technologies, Oxford (UK) 2011-2014.

[S2] W. R. Busing, H. A. Levy, Acta Crystallogr. 1957, 10, 180-182.

[S3] a) G. Sheldrick, SHELXT, University of Göttingen and Bruker AXS GmbH, Karlsruhe (Germany), 2012-2014. b) M. Ruf, B. C. Noll, Application Note SCXRD 503, Bruker AXS GmbH, Karlsruhe (Germany), 2014. c) G. Sheldrick, Acta Crystallogr. A 2015, 71, 3-8.

[S4] M. C. Burla, R. Caliandro, M. Camalli, B. Carrozzini, G. L. Cascarano, L. De Caro, C. Giacovazzo, G. Polidori, D. Siliqi, R. Spagna, J. Appl. Crystallogr. 2007, 40, 609-613.

[S5] a) G. Sheldrick, SHELXL-20xx, University of Göttingen and Bruker AXS GmbH, Karlsruhe (Germany), 2012-2014. b) G. Sheldrick, Acta Crystallogr. A 2008, 64, 112-122.

[S6] L. Ueberricke, D. Holub, J. Kranz, F. Rominger, M. Elstner and M. Mastalerz, Chem. Eur. J. 2019, 25, 11121-11134.

[S7] L. Ueberricke, I. Ciubotaru, F. Ghalami, F. Mildner, F. Rominger, M. Elstner, M. Mastalerz, Chem. Eur. J. 2020, 26, 11634-11642.

[S8] D. Kiefer, R. Kroon, A. I. Hofmann, H. Sun, X. Liu, A. Giovannitti, D. Stegerer, A. Cano, J. Hynynen, L. Yu, Y. Zhang, D. Nai, T. F. Harrelson, M. Sommer, A. J. Moulé, M. Kemerink, S. R. Marder, I. McCulloch, M. Fahlman, S. Fabiano,

C. Müller, Nature Materials 2019, 18, 149-155.

[S9] B. Joo, E.-G. Kim, Chem. Commun. 2015, 51, 15071-15074. 
[S10] M. Elstner, D. Porezag, G. Jungnickel, J. Elsner, M. Haugk, T. Frauenheim, S. Suhai, G. Seifert, Phys. Rev. B 1998, 58, 7260-7268.

[S11] A. Kubas, F. Hoffmann, A. Heck, H. Oberhofer, M. Elstner, J. Blumberger, J. Chem. Phys. 2014, 140, 104105-104121.

[S12] A. Kubas, F. Gajdos, A. Heck, H. Oberhofer, M. Elstner and J. Blumberger, Phys. Chem. Chem. Phys. 2015, 17, 14342-14354.

[S13] A. Heck, J. J. Kranz, T. Kubař, M. Elstner, J. Chem. Theory Comput. 2015, 11, 5068-5082.

[S14] A. Heck, J. J. Kranz, M. Elstner, J. Chem. Theory Comput. 2016, 12, 3087-3096.

[S15] T. Kubař, M. Elstner, J. Royal Soc. Interface 2013, 10, 20130415-20130415.

[S16] T. Kubař, M. Elstner, J. Phys. Chem. B 2010, 114, 11221-11240.

[S17] G. Lüdemann, I. A. Solov'yov, T.Kubař, M. Elstner, J. Am. Chem. Soc. 2015, 137 (3),1147-1156.

[S18] G. Lüdemann, P. B. Woiczikowski, T. Kubař, Marcus Elstner, T. B. Steinbrecher J. Phys. Chem. B 2013, 117 (37), 10769-10778.

[S19] D. Holub, H. Ma, N. Krauß, T. Lamparter, M. Elstner, N. Gillet, Chem. Science 2018, 9, 1259-1272.

[S20] B. Aradi, B. Hourahine, T. Frauenheim, J. Phys. Chem. A 2007, 111, 5678-5684.

[S21] R. G. Kepler, Phys. Rev. 1960, 119, 1226-1229.

[S22] T. Kubař, P. B. Woiczikowski, G. Cuniberti, M. Elstner, J. Phys. Chem. B 2008, 112, 7937-7947.

[S23] L. Zhu, Y. Yi, A. Fonari, N. S. Corbin, V. Coropceanu, J.-L. Brédas, J. Phys. Chem. C 2014, 118, 26, 14150-14156 